Argonne

\title{
Detailed analyses of a TRISO-fueled microreactor
}

Modeling of a Micro-Reactor System using NEAMS tools

Nuclear Science and Engineering Division 


\begin{abstract}
About Argonne National Laboratory
Argonne is a U.S. Department of Energy laboratory managed by UChicago Argonne, LLC under contract DE-AC02-06CH11357. The Laboratory's main facility is outside Chicago, at 9700 South Cass Avenue, Argonne, Illinois 60439. For information about Argonne and its pioneering science and technology programs, see www.anl.gov.
\end{abstract}

\title{
DOCUMENT AVAILABILITY
}

Online Access: U.S. Department of Energy (DOE) reports produced after 1991 and a growing number of pre-1991 documents are available free at OSTI.GOV (http://www.osti.gov/), a service of the US Dept. of Energy's Office of Scientific and Technical Information.

Reports not in digital format may be purchased by the public from the National Technical Information Service (NTIS):

U.S. Department of Commerce

National Technical Information Service

5301 Shawnee Rd

Alexandra, VA 22312

www.ntis.gov

Phone: (800) 553-NTIS (6847) or (703) 605-6000

Fax: (703) 605-6900

Email: orders@ntis.gov

Reports not in digital format are available to DOE and DOE contractors from the Office of Scientific and Technical Information (OSTI):

U.S. Department of Energy

Office of Scientific and Technical Information

P.O. Box 62

Oak Ridge, TN 37831-0062

www.osti.gov

Phone: (865) 576-8401

Fax: (865) 576-5728

This report was prepared as an account of work sponsored by an agency of the United States Government. Neither the United States Government nor any agency thereof, nor UChicago Argonne, LLC, nor any of their employees or officers, makes any warranty, exp ress or implied, or assumes any legal liability or responsibility for the accuracy, completeness, or usefulness of any information, apparatus, product, or process disclosed, or represents that its use would not infringe privately owned rights. Reference herein to any specific commercial product, process, or service by trade name, trademark, manufacturer, or otherwise, does not necessarily constitute or imply its endorsement, recommendation, or favoring by the United States Government or any agency thereof. The views and opinions of document authors expressed herein do not necessarily state or reflect those of the United States Government or any agency the reof, Argonne National Laboratory, or UChicago Argonne, LLC.
} 


ANL/NEAMS-21/3

\section{Detailed analyses of a TRISO-fueled microreactor}

Modeling of a Micro-Reactor System using NEAMS tools

prepared by

N. Stauff, K. Mo, Y. Cao, J. Thomas, Y. Miao, L. Zou, D. Nunez, E. Shemon, B. Feng, K. Ni Argonne National Laboratory

September 30, 2021 



\section{EXECUTIVE ABSTRACT}

Within the U.S. Department of Energy (DOE) Office of Nuclear Energy's NEAMS program, the Application Drivers Technical Area has been tasked to demonstrate the ability of the NEAMS tools to perform high-fidelity and multiphysics simulations on nuclear microreactors. These are designed by various vendors and U.S. government programs to power remote communities or industrial sites. Microreactors are challenging traditional tools due to their reliance on innovative technologies and their flexible operation requirements. The advanced codes developed under the NEAMS program are meant to provide the capability to perform coupled multiphysics transient simulations enabling reactor designers to optimize microreactor performance under a wide range of potential operating conditions.

One of the major outcomes of the NEAMS Application Drivers area is to provide userassessment of various NEAMS codes and perform single-physics and coupled simulations on a micro-reactor design. This includes Griffin for neutronics, BISON for thermo-mechanics, Sockeye for heat pipe performance modeling, SAM for system-level thermal hydraulics modeling, and SWIFT for hydrogen modeling in hydride metal. All codes were developed within the MOOSE (Multiphysics Object-Oriented Simulation Environment) framework. This project focused on training a diverse team of experts in their fields on these codes and gathering user feedback in the first half of FY21 and applying them to micro-reactor analyses in the second half of FY21. The work discussed in this report focused on modeling the Argonnedesigned heat pipe micro-reactor (HP-MR), which is a TRISO-fueled design developed as a modeling experiment using coupled Griffin, Sockeye, SAM, and BISON codes for high-fidelity multiphysics transient simulations.

Several analyses performed in this project are employing newly developed codes and methods that are applied for the first time by non-developers. The motivation is both to demonstrate and showcase those capabilities, while building user experience within the laboratory and providing feedback to the users. For instance, to our knowledge, it is the first time the Griffin "Diffusion + SPH" method was applied for a heterogeneous geometry model in multiphysics transient simulations using bidimensional cross-sections tables. Sockeye was applied for the first time this year by the Application Driver team in multiphysics simulations. Finally, different BISON simulations are performed for the first time by this Application Driver team on both small-scale simulation for a single TRISO fuel particle, and a full-scale simulation for a full core microreactor.

Our analysis provides preliminary confirmation of the feasibility of applying the NEAMS codes for multiphysics simulations to model load following transients and accidental scenarios. Detailed demonstration was performed on a simple unit-cell model to perform transient simulations, while verifying accuracy of the multiphysics solution obtained with the NEAMS codes. Results on the full core focused on showcasing the multiphysics workflow rather than detailed verification of the results, at least for this report.

On this unit-cell model, the Griffin "diffusion + SPH" method was applied to improve the accuracy of the reactor physics results compared to those from diffusion without SPH. With this method, not only is the fast convergence rate of the diffusion method preserved in the multiphysics simulations, but the axial power distributions and reactivity temperature feedback effects have also been calculated accurately. The $\mathrm{S}_{\mathrm{N}}$ approach was also successfully applied, 
but further computational performance improvement is needed to enable its application to unitcell and full-core multiphysics modeling problems. Verification of the Griffin cross-section interpolation methodology used in multiphysics simulations was completed. This work demonstrated that Griffin accurately interpolates cross-sections based on two varying parameters such as the fuel temperature, moderator temperature, and the hydrogen content in the moderator. Multiphysics load-following transients were completed by coupling Griffin, BISON, and Sockeye through the MultiApp approach in MOOSE. This analysis showcased the impact of these different model improvements on the accuracy of the transient simulations.

Based on the success of the unit cell simulation, the multiphysics simulations were expanded to the full-core geometry. At this time, the Griffin diffusion method (without SPH correction) was used in the full-core multiphysics simulations to demonstrate the workflow for transient simulations. BISON's thermal-mechanical modeling capability coupled with heat conduction and elastic mechanics was demonstrated on the full-core simulation, while only the thermalmechanical model was used for transients analysis. The effective conduction model of Sockeye was used to model heat transfer through the heat-pipe in our multiphysics simulations, supported by estimates of the thermal conductivity of the vapor core generated with the twophase models. The multiphysics (BISON/Sockeye) models developed in this work were made available through the NRIC Virtual Test Bed (VTB) repository to reduce the learning curve for future and existing users of NEAMS tools. Both unit-cell and full-core load following transients display the self-regulation capability of the HP-MR where a drop in heat-removal rates from the condenser side of the heat-pipe was quickly followed by a power reduction with less than $50 \mathrm{~K}$ of peak fuel temperature variations.

Aside from the load following power transient, a single heat pipe failure power transient scenario was also simulated demonstrating that the multiphysics simulation approach coupling Griffin/BISON/Sockeye is capable of predicting heat pipe cascade failure events. In addition, multiphysics transients were modeled under a decay heat removal scenario using SAM/MOOSE coupling through the MultiApp approach to model heat removal from the reactor vessel through the RCCS system during accident scenarios. Finally, a side study was completed to assess the capability of BISON to model TRISO fuel particles for microreactor applications.

In conclusion, the MOOSE MultiApps framework and NEAMS codes were demonstrated to be capable of design and safety analysis applications for heat-pipe micro-reactors. Several ongoing improvements in the NEAMS codes will enable more accurate dynamics modeling of microreactors.

For future work, improved Griffin solutions of our full-core model will be assessed using $\mathrm{S}_{\mathrm{N}}$ methods or the Diffusion with SPH approach. To reduce computational cost of neutronic simulations during multiphysics transients, a point kinetics model within Griffin or SAM can be utilized. Accurate load-following modeling of such thermal-spectrum micro-reactors should include a depletion model to account for Xenon poisoning. Modeling hydride metal moderator capability to retain hydrogen throughout temperature transients will be performed using the SWIFT code under development at LANL. Finally, by leveraging the experience and capabilities gained in this project, the KRUSTY test microreactor will be modeled to provide experimental validation of NEAMS multiphysics simulations. 


\section{TABLE OF CONTENTS}

EXECUTIVE ABSTRACT

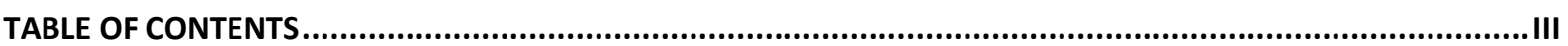

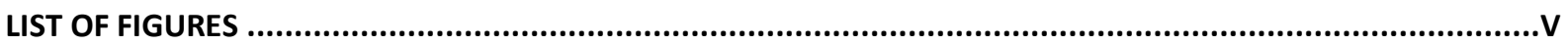

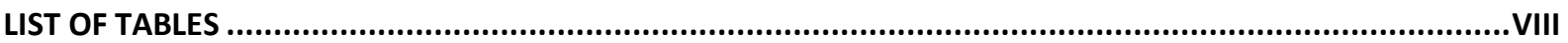

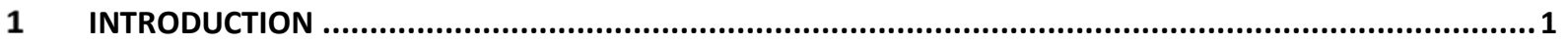

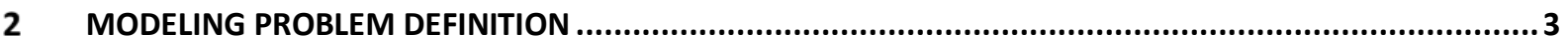

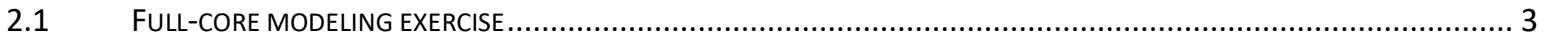

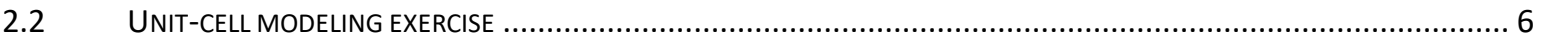

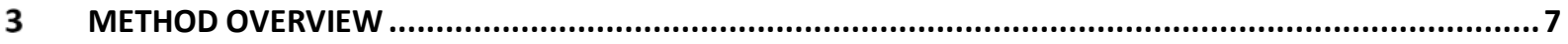

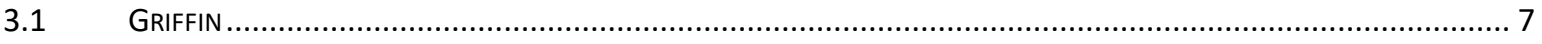

3.2 BISON ………………… 7

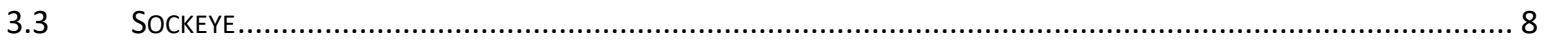

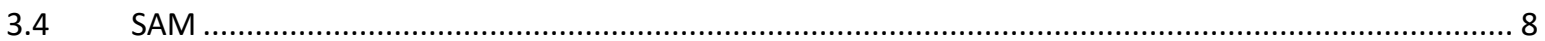

$4 \quad$ NEUTRONICS ANALYSIS OF MICROREACTOR UNIT-CELL MODEL ................................................... 10

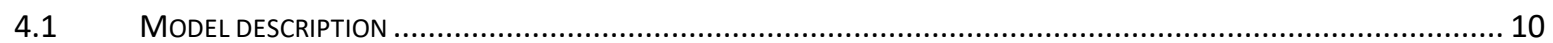

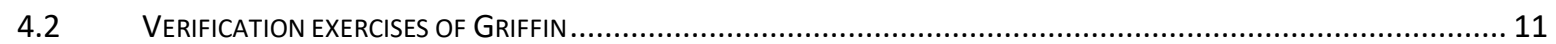

4.2.1 Comparison of Serpent-2 solutions with various Griffin solvers ................................................. 12

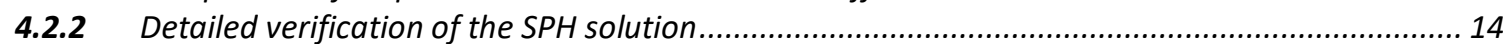

4.3 GENERATION AND VERIFICATION OF BIDIMENSIONAL TABULATED CROSS-SECTIONS FOR GRIFFIN............................ 16

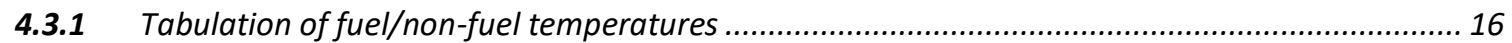

4.3.2 Tabulation of fuel temperature and $\mathrm{H}$-content.................................................................... 17

4.3.3 Verification of the accuracy of the interpolation of bidimensional tabulated cross section .......... 18

4.4 GRIFFIN REACTOR PHYSICS MODEL WITH SWIFT-GENERATED VARYING H-CONTENT ......................................... 21

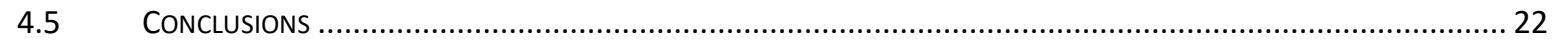

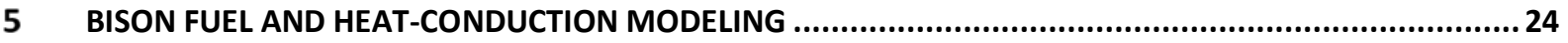

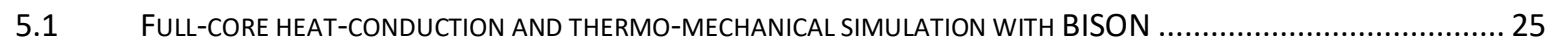

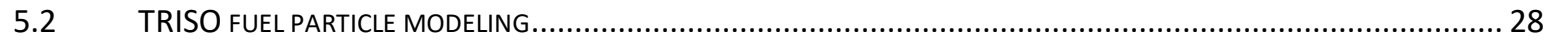

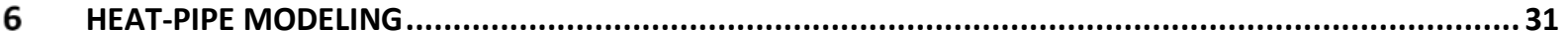

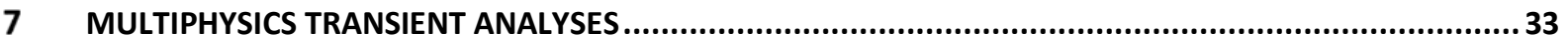

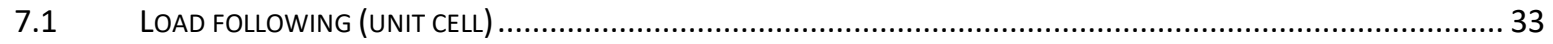

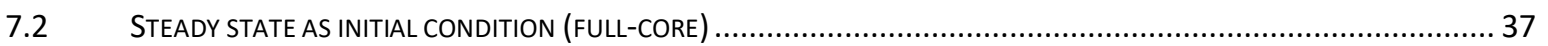

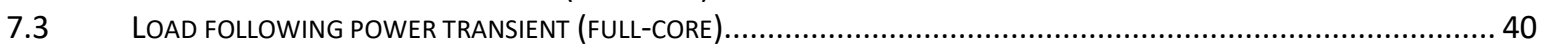

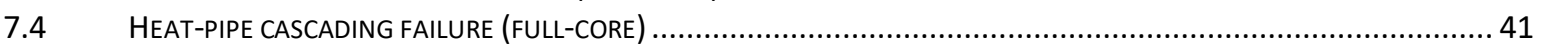

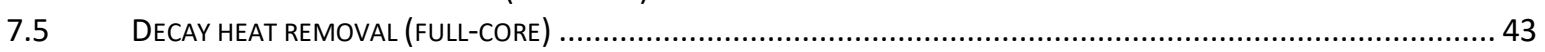

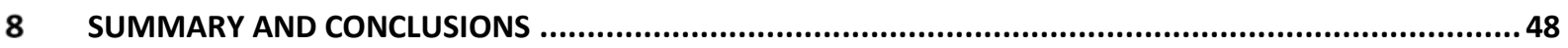

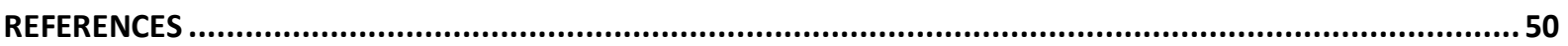

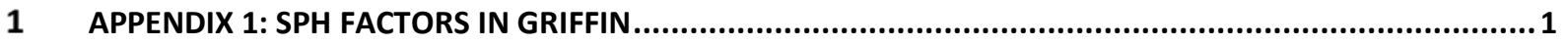

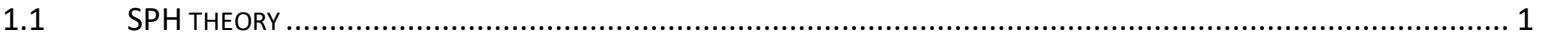

1.2 SPH METHOD PROCEDURE IN GRIFFIN AND VERIFICATION OF SPH FACTORS FOR THE UNIT-CELL MODEL .................... 2

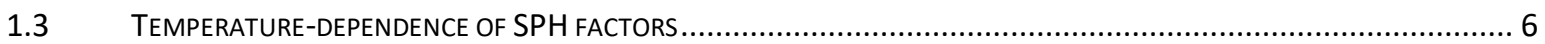

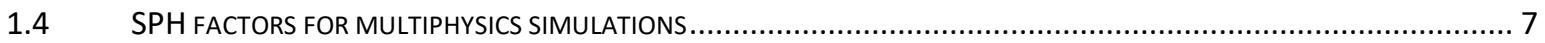




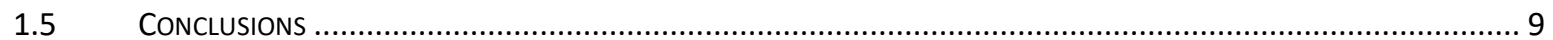

2 APPENDIX 2: GRIFFIN CROSS-SECTIONS GENERATION AND INTERPOLATION................................. 10

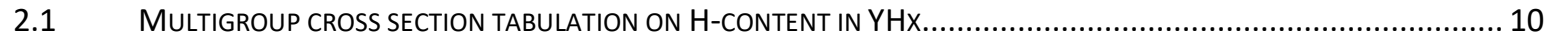

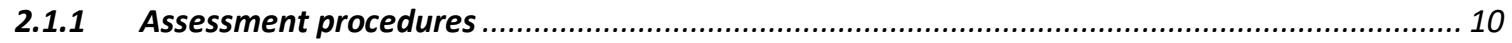

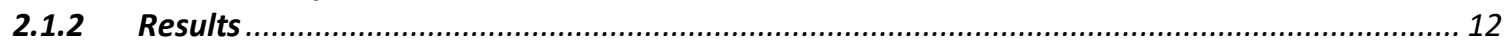

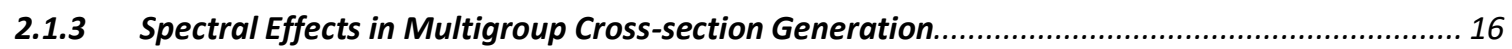

2.2 Heterogeneity EfFeCtS In Multigroup Cross-SECTION Generation..................................................... 17

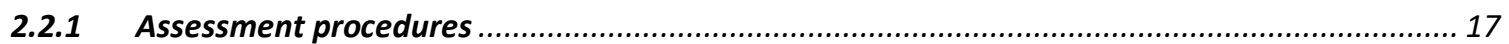

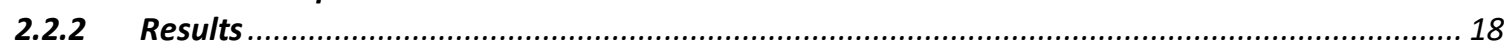

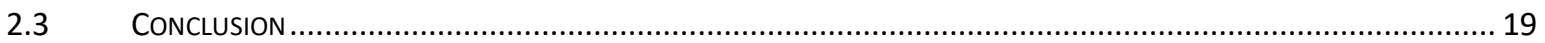




\section{LIST OF FIGURES}

Figure 2-1. Radial and axial layout of the HP-MR .......................................................... 3

Figure 2-2. Radial layout of fuel assembly of the HP-MR. .................................................... 4

Figure 2-3. Zoom-in on the axial description of the upper/lower plugs for the moderator and

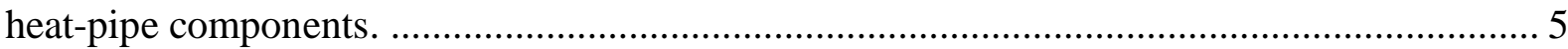

Figure 2-4. Axial/radial layout of unit-cell assembly. .......................................................... 6

Figure 4-1. Serpent-2 calculated reference k-effs of the 3-D unit cell with its fuel and moderators at different temperatures. ............................................................................. 11

Figure 4-2. Calculated reactivity temperature feedback coefficients for the 3-D unit-cell

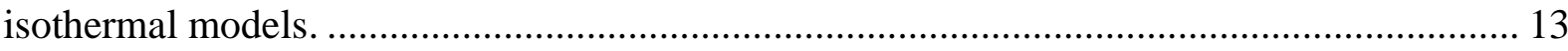
Figure 4-3. Calculated axial power distributions for the 3-D unit-cell isothermal model at 800 K.

Figure 4-4. Calculated k-effs for the 3-D unit-cell isothermal model at different temperatures using the diffusion and SPH method.

Figure 4-5. Calculated k-effs for the 3-D unit-cell at different temperatures using 2d-grid cross section table in the diffusion and SPH method compared with the Serpent-2 reference results.

Figure 4-6. Calculated k-effs from Serpent-2 models at different temperatures and different hydrogen concentrations.

Figure 4-7. Comparison of the Griffin SAAF-CFEM-SN calculation with multigroup cross sections directly converted from Serpent-2 output or with multigroup cross sections interpolated from the 2-d look-up table a) k-effs for all $\mathrm{x}$ cases at $700 \mathrm{~K}$ and $1000 \mathrm{~K} \mathrm{~b}$ ) deviation in $\mathrm{K}$-eff for all these cases c) normalized axial distributions for the case with $\mathrm{T}=1000 \mathrm{~K}$ and $\mathrm{x}=1.1 \mathrm{~d}$ ) maximum relative differences of axial power distributions for all cases.

Figure 4-8. a) Hydrogen content throughout the axial direction in the $\mathrm{YH}_{2}$ moderator pin as estimated by SWIFT, b) Griffin SN $(3,3)$ calculated axial power distributions for the 3-D unit cell models with fuel temperature at $852 \mathrm{~K}$ and with $\mathrm{H}$-content in the $\mathrm{YHx}$ moderator: $\mathrm{x}=2.0$ or SWIFT calculated $\mathrm{x}$; and the relative differences between the two distributions. .............. 22 Figure 5-1. Various meshes used in different simulation cases: (a) heat conduction full-core simulation; (b) thermo-mechanical full-core simulation; and (c) 2D-RZ standalone TRISO fuel particle simulation.

Figure 5-2. Temperature evolution of the microreactor core to establish a steady state: 3D temperature distribution (unit: K) at (a) time $=1 \mathrm{sec}$; (b) time $=10 \mathrm{sec}$; and (c) time $=60 \mathrm{sec}$; 2D temperature distribution (unit: K) at the core center at (d) time $=1 \mathrm{sec}$; (e) time $=10 \mathrm{sec}$; and (f) time $=60 \mathrm{sec}$.

Figure 5-3. Temperature evolution of the microreactor core to establish a steady state: (a) average fuel temperature, and (b) average heat pipe surface temperature.

Figure 5-4. Displacement and temperature evolution of the microreactor core during establishing the steady state: 3D displacement distribution (unit: $\mathrm{m}$ ) at (a) time $=1 \mathrm{sec}$; (b) time $=10 \mathrm{sec}$; and (c) time $=60 \mathrm{sec}$; and 3D temperature distribution (unit: K) at (d) time $=1$

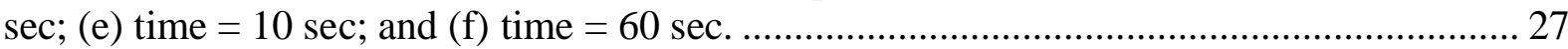
Figure 5-5. Graphic demonstrations of displacement near the top of reflector region. ........... 28 
Figure 5-6. Temperature distribution of a TRISO fuel particle in the microreactor at (a) time = 0 , and (b) time $=10$ years. The "3D" images were produced based on the $2 \mathrm{D}-\mathrm{RZ}$ simulation results for demonstration.

Figure 5-7. Computed histories of (a) fission gas release and particle pressure and (b) maximum tangential stress of IPyC, OPyC and $\mathrm{SiC}$. End of irradiation (10 years) corresponds to a burnup of $\sim 17 \mathrm{MWd} / \mathrm{kgHN}$.

Figure 6-1. (a) Schematic of heat pipe model with boundary conditions (not to scale), (b) operating limits of heat pipe as computed by Sockeye utility. 32

Figure 6-2. (a) Axial void fraction profile and (b) temperature profiles predicted by Sockeye at stead-state, normal operating conditions by the two-phase model................................... 32

Figure 7-1. Typical MultiApp strategy for micro-reactor multiphysics simulations.............. 33 Figure 7-2. A typical steady state reactor physics (total neutron flux) and heat conduction simulation result (material temperature) of multiphysics simulation.

Figure 7-3. Time-evolution of the total unit cell power during load-following power transient

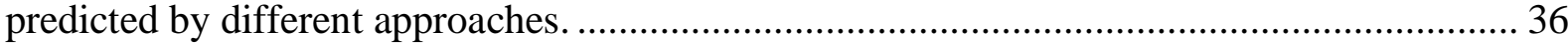
Figure 7-4. Time-evolution of the average fuel temperature during load-following power transient predicted by different approaches.

Figure 7-5. Temperature distribution of the 1/6-core at steady state based on the Griffin-

BISON-Sockeye simulation with thermal only BISON approach.................................... 38 Figure 7-6. (a) Displacement field and (b) local von Mises stress of the monolith matrix due to thermal expansion of the 1/6-core at steady state based on the Griffin-BISON-Sockeye simulation with thermo-mechanics BISON approach..................................................... 39 Figure 7-7. Time evolution of fuel temperature and power during the load following power transient.

Figure 7-8. The snapshots of temperature profile around the maximum temperature (left) and new-steady-state (right) time point after the load following power transient.

Figure 7-9. Heat pipe cascading failure after a single heat pipe failure (highlighted by a yellow circle) in the $1 / 6$-core operating at $607,000 \mathrm{~W}$ 42

Figure 7-10. Simulation results of the single heat pipe failure in the 1/6-core operating at $345,600 \mathrm{~W}$ showing absence of heat pipe cascading failure.......................................... 43 Figure 7-11. Schematic of the simplified RCCS model applied to a simple hexagonal block. 44 Figure 7-12. Initial temperature distribution (left) and power distribution (right) of the 1/6-

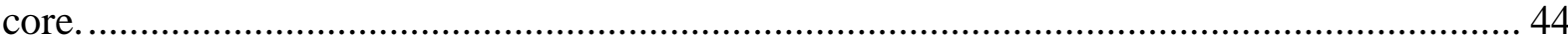

Figure 7-13. Wigner-Way and ANS-94 decay heat curves............................................ 45 Figure 7-14. Power density distribution at two instances during the simulated transient....... 46 Figure 7-15. Temperature distribution at two instances during the simulated transient. ........ 46 Figure 7-16. Maximum fuel temperature during transient............................................. 47 Figure A1-1. Axial power distributions calculated from Serpent-2 model, the diffusion method, and the diffusion and SPH method for the 3-D unit cell model at fuel and moderator temperature at $800 \mathrm{~K}$.

Figure A1-2. SPH equivalence zone map: Left- 10 axial equivalence zone; Right - 40 axial equivalence zone.

Figure A1-3. Normalized axial total flux distributions calculated from the Serpent-2 model, the diffusion and SPH method with 10 axial equivalence zone, the diffusion and SPH method with 40 axial equivalence zone for the 3-D unit cell model at fuel and moderator temperature at $600 \mathrm{~K}$. 
Figure A1-4. Calculated a) axial power density distributions and b) axial averaged fuel temperature distributions in the 3-D unit cell at steady state from the multiphysics simulations with the diffusion and SPH method using 10 axial equivalence zones or 40 axial equivalence zones

Figure A1-5. Griffin calculated SPH factors for the unit-cell model at temperature grid points of $600 \mathrm{~K}, 700 \mathrm{~K}, 800 \mathrm{~K}, 1000 \mathrm{~K}$ and $1200 \mathrm{~K}$ and energy group: a) [1.0E-5, 8.0E-2] eV; b) $[0.625,1.3] \mathrm{eV}$; c) [4.0, 148.73] eV; d) [1.35, 40] MeV.

Figure A1-6. Comparison of the calculated k-eff from Griffin using the diffusion and SPH method with interpolated cross sections and SPH factors with Serpent-2 reference result...... 7 Figure A1-7. a) The maximum differences among the calculated axial power densities using the direct cross sections and SPH factors or using the interpolated cross sections and SPH factors; b) normalized axial power density distributions for the 3-D unit cell model at $1000 \mathrm{~K}$.

Figure A2-1. Method overview of bidimensional temperature \& H-content cross-section interpolation verification (Left) and Serpent-2 results for all grid points (Right). ................ 11

Figure A2-2. Selection of interpolation set from the master cross-section library................ 12 Figure A2-3. Eigenvalue comparison of the Griffin calculation with cross-sections directly converted from Serpent-2 output or with cross-sections interpolated from the 2-D look-up table: (a) keff for all grid points; (b) $\Delta k$ eff $=k$ effdirect $-x s-k$ effinterpolated $-x s$ for temperature grid at $600 \mathrm{~K}, 800 \mathrm{~K}$ and $1200 \mathrm{~K}$; (c) $\Delta k$ eff for temperature grid at $700 \mathrm{~K}$ and $1000 \mathrm{~K}$.

Figure A2-4. Axial power comparison of the Griffin calculation with cross-sections directly converted from Serpent-2 output or with cross-sections interpolated from 2-D look-up table: a) Serpent- 2 normalized axial power compared with Griffin power at $\mathrm{T}=1200 \mathrm{~K}$ and $\mathrm{x}=1.4$; b) normalized axial distributions for case with $\mathrm{T}=1000 \mathrm{~K}$ and $\mathrm{x}=1.1$; c) maximum relative differences of axial power for temperature grid at $600 \mathrm{~K}, 800 \mathrm{~K}$ and $1200 \mathrm{~K}$; d) maximum relative differences of axial power for temperature grid at $700 \mathrm{~K}$ and $1000 \mathrm{~K}$. 16 Figure A2-5. Comparison of the calculated Griffin $\mathrm{S}_{\mathrm{N}}(3,3)$ k-effs using one region homogenized cross-sections or multiple region homogenized cross-sections with the Serpent2 reference results with fuel material at $600 \mathrm{~K}$ in the 3-D unit cell.................................... 17 Figure A2-6. Interpolation of varied material cross-section assessment cases settings.......... 18 Figure A2-7. Axial power distribution in the fuel pin comparison between direct cross-section and interpolated cross-section calculation of case \#1, case \#2 (Left) and case \#3 (Right)..... 19 


\section{LIST OF TABLES}

Table 2-1. Detailed reactor description - cold temperature conditions. ................................... 5

Table 2-2. Heat-pipe description - hot temperature conditions. ......................................... 6

Table 4-1. The upper energy boundaries $(\mathrm{MeV})$ for the 11-group neutron energy structure for Griffin reactor physics calculation............................................................................. 10

Table 4-2. Serpent-2 calculated reference k-effs of the 3-D unit cell isothermal models. ...... 11 Table 4-3. Griffin calculated k-effs of the 3-D unit cell isothermal models using Diffusion, the SAAF-CFEM-SN and DFEM-SN schemes. $\triangle \mathrm{k}$-eff is the difference between Griffin and

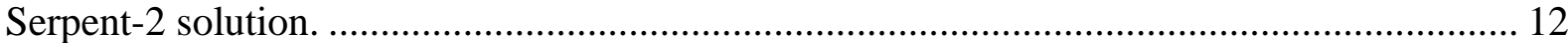
Table 4-4. Difference in k-effs [pcm] with the direct or interpolated cross sections and SPH factors.

Table 6-1. Parameters in sockeye heat pipe performance model........................................ 31 Table A1-1. Calculated k-effs of the 3-D unit cell isothermal models from Serpent-2 and from diffusion and SPH method.

Table A1-2. Calculated $\Delta \mathrm{k}$-effs from diffusion and SPH method with the direct or interpolated cross sections and SPH factors for the 3-D unit cell model.

Table A1-3 Calculated temperature feedback coefficients $(\mathrm{pcm} / \mathrm{K})$ from Griffin diffusion and SPH method using cross sections directly from Serpent output or using the interpolated cross sections for the 3-D unit cell model.

Table A2-1. Calculated fuel temperature reactivity feedback coefficients ( $\mathrm{pcm} / \mathrm{K}$ ) and the reactivity feedback coefficient due to the $H$-content variation $(\mathrm{pcm} /(\Delta \mathrm{x} / \mathrm{x}))$ from Griffin $\mathrm{S}_{\mathrm{N}}(3,3)$ method using cross sections directly from Serpent output or using the interpolated cross sections for the 3-D unit cell model.

Table A2-2. Result comparisons between interpolated cross-section approach and direct crosssection approach for three cases 


\section{Introduction}

Nuclear micro-reactors are designed to power remote communities or industrial sites [1] by various vendors and U.S. government programs. These reactors typically employ technologies from Very High Temperature Reactor (VHTR) designs or reactor concepts cooled by heat pipes. The innovative design features employed by these concepts motivate the assessment of a modern suite of advanced modeling tools as that developed by the Nuclear Energy Advanced Modeling and Simulation (NEAMS) program [2]. Beyond permitting design and analysis of these reactors via traditional single-physics tools, such advanced tools provide the capability to perform coupled multiphysics transient simulations enabling reactor designers to optimize micro-reactor performance under a wide range of potential operating conditions.

Within the U.S. Department of Energy (DOE) Office of Nuclear Energy's NEAMS program, the micro-reactor activity within the Application Drivers Technical Area is assessing the ability of NEAMS tools to perform high-fidelity and multiphysics simulations on representative microreactor problems to support the aforementioned development efforts. This project aims at leveraging the work completed by other groups at the Idaho, Argonne, and Los Alamos National Laboratories (INL, ANL, LANL) [3, 4, 5]. One of the major outcomes of the NEAMS Application Drivers area is to provide user-assessment of various NEAMS codes and perform single-physics and coupled simulations on the modeling problems based on a micro-reactor design. This includes Griffin [6] for neutronics, BISON [7] for thermo-mechanics, Sockeye [8] for heat pipe performance modeling, SAM [10] for system-level thermal hydraulics modeling, and SWIFT [11] for hydrogen modeling in hydride metal. All codes were developed within the MOOSE (Multiphysics ObjectOriented Simulation Environment) [12,13] framework.

Several analyses performed in this project are employing newly developed codes and methods that are applied for the first time by non-developers. The motivation is both to demonstrate and showcase those capabilities, while building user experience within the laboratory and providing feedback to the users. For instance, to our knowledge, it is the first time the Griffin "Diffusion + SPH" method was applied for a heterogeneous geometry model in multiphysics transient simulations using bidimensional cross-sections tables. Sockeye was applied for the first time this year by the Application Driver team in multiphysics simulations. Finally, different BISON simulations are performed for the first time by this Application Driver team on both small-scale simulation for a single TRISO fuel particle, and a full-scale simulation for a full core microreactor.

In previous work [14], our team has initiated a representative modeling problem featuring a $2 \mathrm{MW}$ thermal micro-reactor core configured with heat pipes and TRISO fuel, and completed neutronicsthermo-mechanical multiphysics simulations on a unit-cell and full-core problems. Effort in FY21 focused on continuing capability assessment. A significant focus of our project, especially in the first part of FY21, was to provide timely user feedback to the code developers. This specific report focuses on the demonstration of the NEAMS tools capability to solve micro-reactor modeling problems.

Section 2 describes the TRISO-fueled heat pipe micro-reactor (HP-MR) design that was developed at Argonne as a modeling exercise for this project, combining various modeling challenges from different concepts of interest to the U.S. industry. The NEAMS codes and tools used in this project are briefly described in Sections 3. Section 4 summarizes the neutronic analysis performed on the unit-cell modeling problem performed with Griffin, while Appendix 1 and 2 provide more detailed 
analyses. Section 5 describes the fuel and thermo-mechanical modeling studies performed with BISON. Section 6 provides a brief description of the heat-pipe modeling analysis performed with Sockeye. Section 7 applies the tools and methods previously described for multiphysics transients simulations. Finally, the conclusions of this study are summarized in Section 8. 


\section{Modeling Problem Definition}

As a modeling exercise, a micro-reactor concept was designed at ANL to gather some of the most pressing modeling challenges faced by the micro-reactor industry: a) the use of heat pipe technologies to remove the nuclear heat; b) the use of Tri-structure ISOtropic (TRISO) fuel to enable operations at very high temperatures; c) the use of rotating control rod drums in the radial reflectors. A full core model of this type of reactor requires different levels of heterogeneity which are usually difficult to capture accurately. Two 3-D modeling problems are proposed in this work based on this micro-reactor concept. The full-core modeling exercise is described in Section 2.1, while a simpler 3-D unit-cell problem is proposed as described in Section 2.2.

\subsection{Full-core modeling exercise}

This Heat Pipe Micro Reactor (HP-MR) core has a rated power of $2 \mathrm{MWt}$ and its radial layout is shown in Figure 2-1, while the fuel assembly is shown in Figure 2-2. A traditional TRISO fuel with 19.95 at\% Low-Enriched Uranium (LEU) in UCO form was adopted in a hexagonal graphite matrix with a $40 \%$ packing fraction. The core length is set to $160 \mathrm{~cm}$ with $20 \mathrm{~cm}$ upper and lower axial reflectors made of beryllium metal. 30 fuel assemblies are surrounded by one ring of beryllium reflector and 12 control drums. This concept employs heat pipes with a thin stainless-steel envelope and potassium heat-transfer fluid. The heat-pipe properties used for Sockeye simulations is further detailed in Section 6.

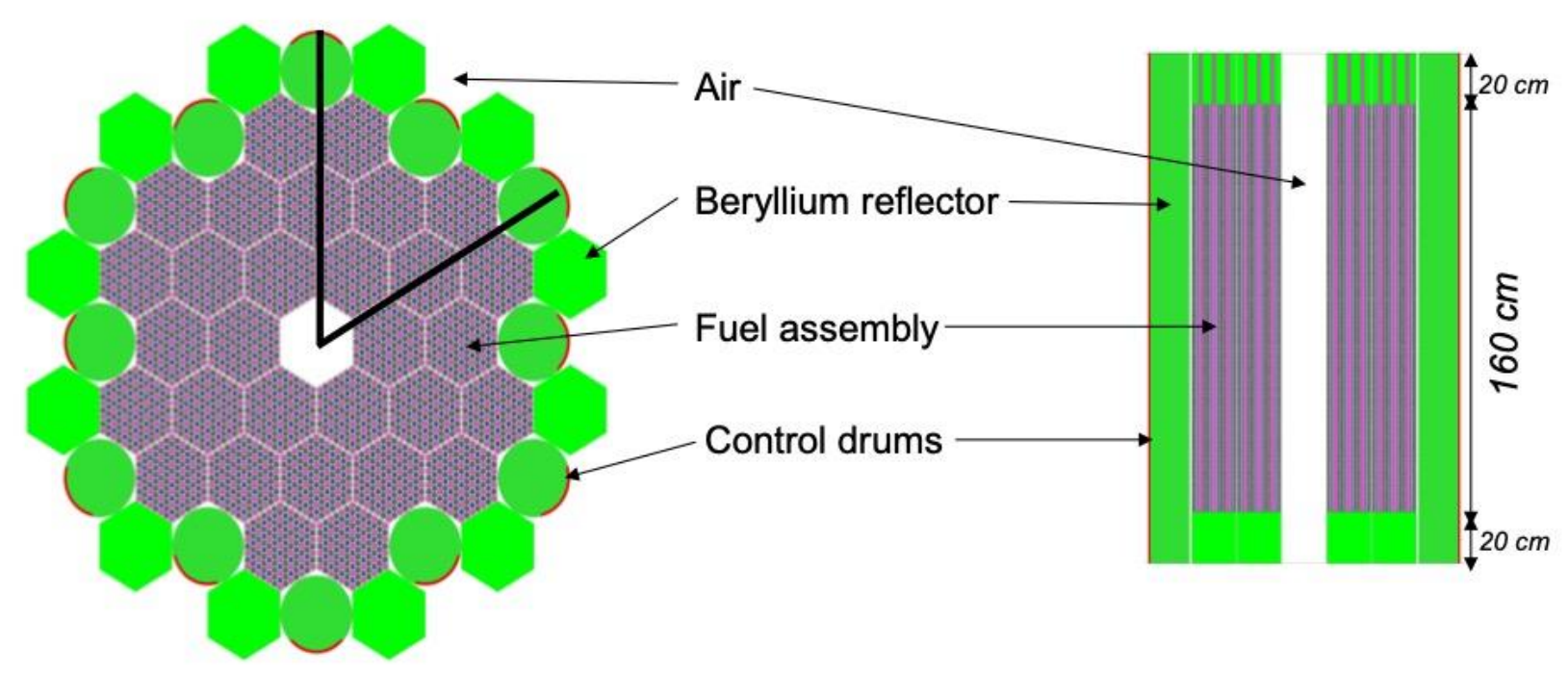

Figure 2-1. Radial and axial layout of the HP-MR.

The main cold-dimensions and characteristics of the different assembly components are described in Table 2-1 and specific dimensions are provided in Table 2-2 for the heat-pipe components. It should be specified that heat-pipe dimensions are provided at hot-temperature conditions since Sockeye cannot be used at this time to perform thermal-expansion calculations within the heatpipe. The TRISO fuel particles are modeled to be packed in the fuel pins with the particle centroids determined in one random realization.

The radial layout of the fuel assembly is provided in Figure 2-2. To achieve an optimum level of moderation, yttrium-hydride $\left(\mathrm{YH}_{2}\right)$ pins are employed in addition to graphite structure component 
as $\mathrm{YH}_{2}$ provides more efficient neutron slowing-down capability enabling the design of more compact core. The yttrium-hydride is surrounded in two layers of thin helium gaps separating the moderator, the stainless steel envelope and the graphite monolith. The heat pipe region has been divided into three zones representing the fluid at the phase of vapor, liquid and wick respectively. The heat pipe is wrapped by the stainless-steel envelope and is separated from the graphite monolith by another layer of helium gap. The axial location of the steel plugs for the moderator and heatpipe components are described in Figure 2-3.

The control system of the core includes 12 control drums located in the radial reflector that are capable of bringing the core to cold shutdown throughout the operation of the reactor. For redundancy purposes, a shutdown rod is located in the central core location.

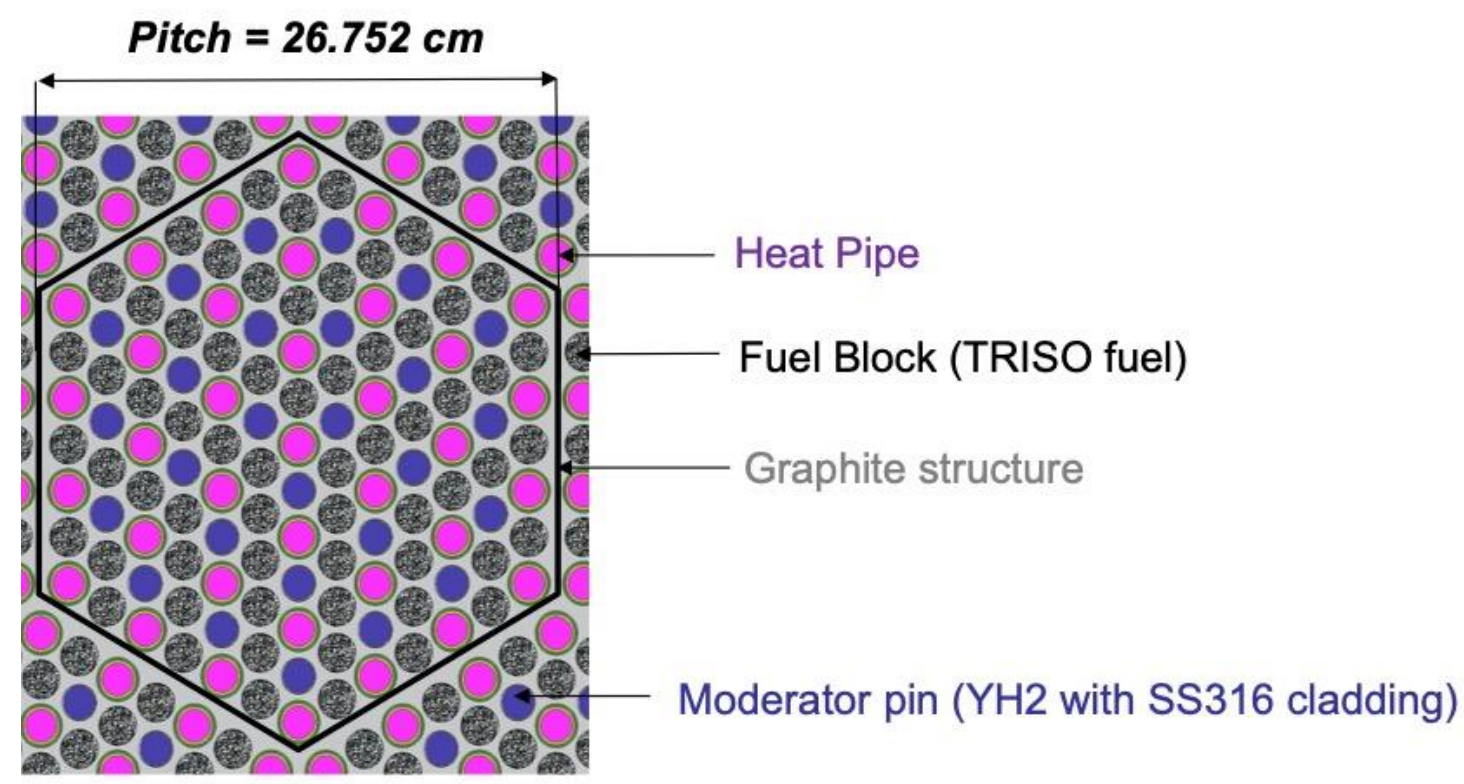

lattice pitch $=2.3 \mathrm{~cm}$

Figure 2-2. Radial layout of fuel assembly of the HP-MR. 


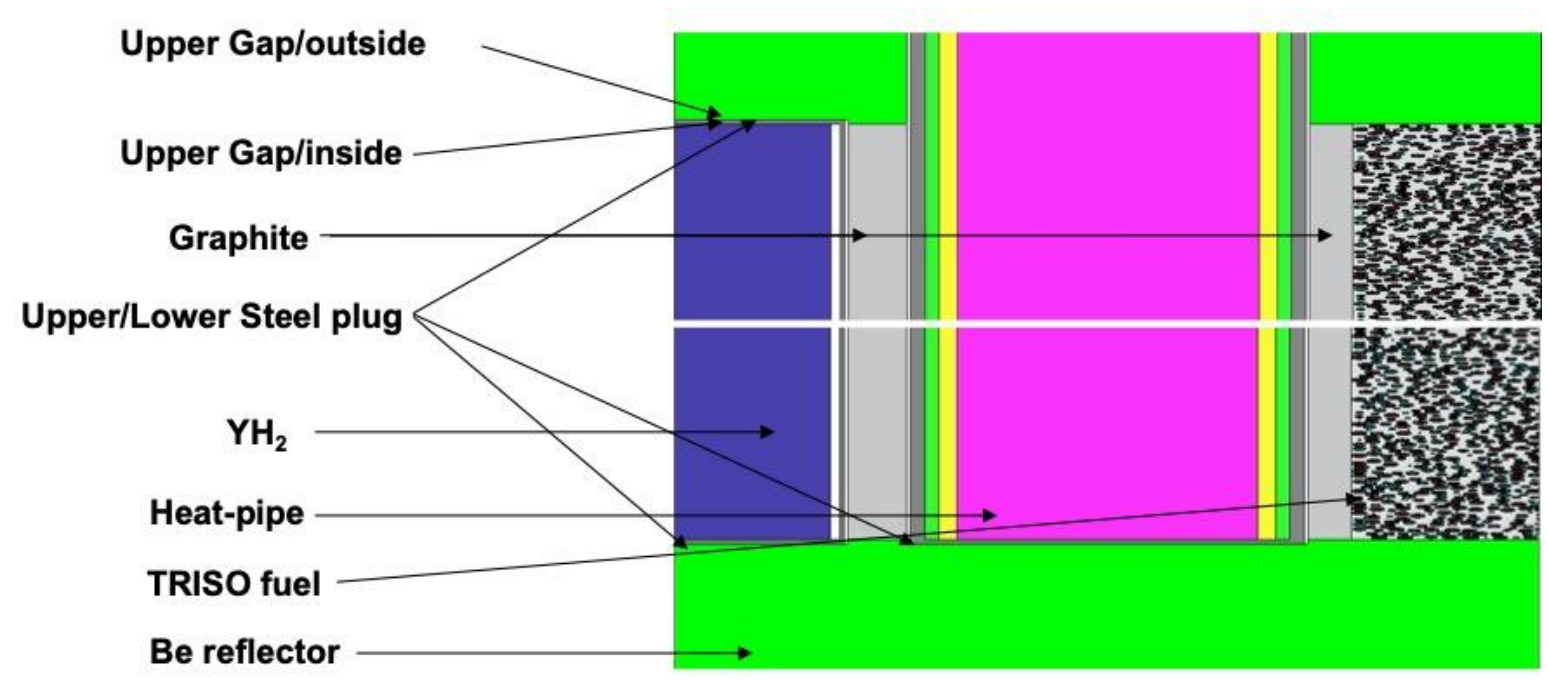

Figure 2-3. Zoom-in on the axial description of the upper/lower plugs for the moderator and heatpipe components.

Table 2-1. Detailed reactor description - cold temperature conditions.

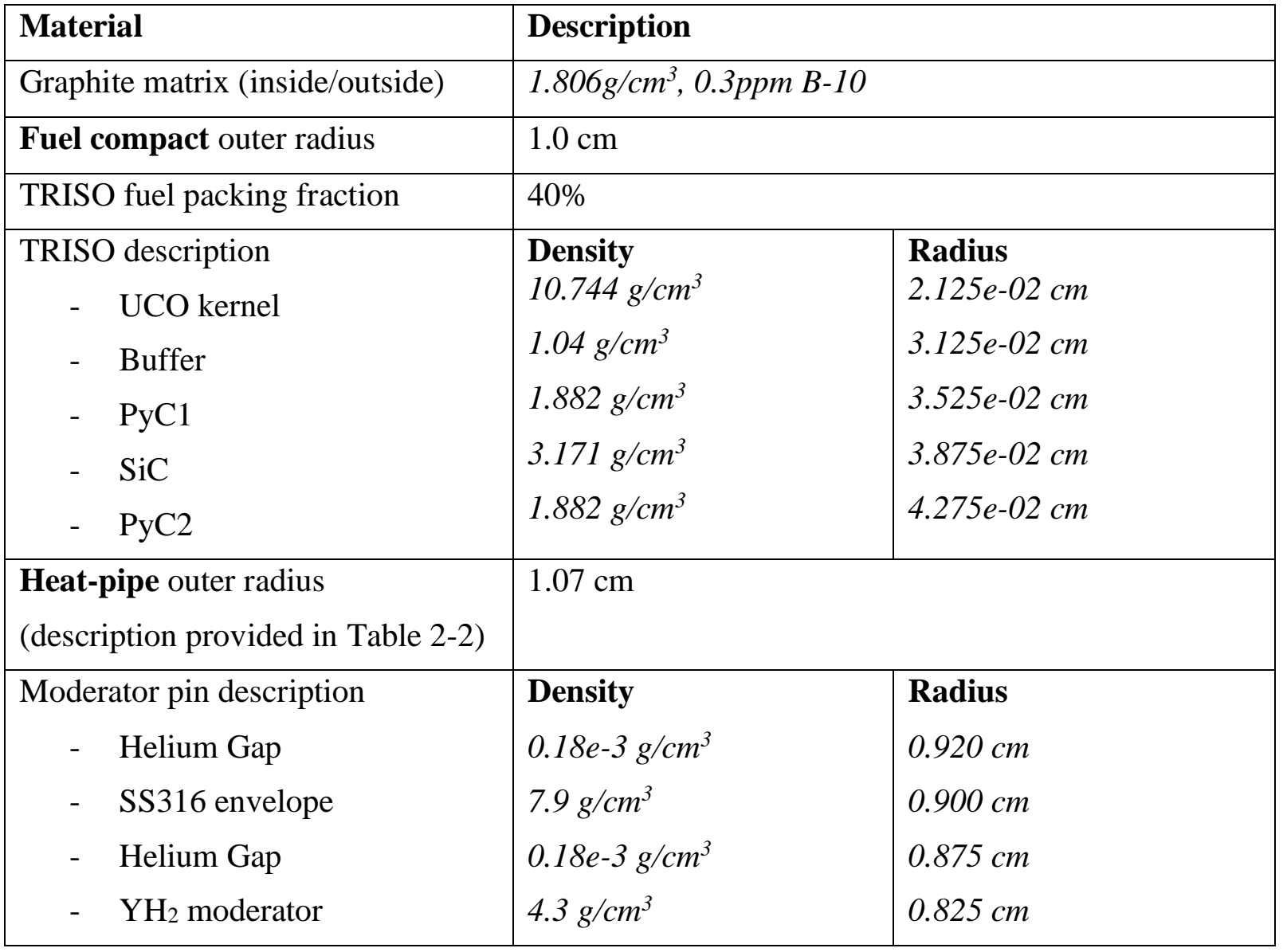


Table 2-2. Heat-pipe description - hot temperature conditions.

\begin{tabular}{|l|l|l|}
\hline Material & Density & Radius \\
\hline He Gap & $0.18 e-3 \mathrm{~g} / \mathrm{cm}^{3}$ & $1.07 \mathrm{~cm}$ \\
\hline SS316 envelope & $7.67 * \mathrm{~g} / \mathrm{cm}^{3}$ & $1.05 \mathrm{~cm}$ \\
\hline K layer & $0.705 \mathrm{~g} / \mathrm{cm}^{3}$ & $0.97 \mathrm{~cm}$ \\
\hline $\begin{array}{l}\text { Wick: } \\
\text { (70vol\% } \mathrm{K}-30 \text { vol\%SS316) }\end{array}$ & $2.753 \mathrm{~g} / \mathrm{cm}^{3}$ & $0.90 \mathrm{~cm}$ \\
\hline K void & $1.11 e-4 \mathrm{~g} / \mathrm{cm}^{3}$ & $0.80 \mathrm{~cm}$ \\
\hline
\end{tabular}

* The current BISON model assumes cold-temperature density for the SS316 envelope as it is modeled outside the Sockeye mesh. This minor discrepancy between neutronics and thermomechanical model will be addressed in the next steps.

\subsection{Unit-cell modeling exercise}

A simpler unit-cell described in Figure 2-4 is used in this report. This unit-cell models one single heat pipe removing $1.8 \mathrm{~kW}$ surrounded by three fuel pins and three moderator pins with reflective boundary conditions. The materials and dimensions of each component of this unit-cell are described in previous section. This unit-cell problem is extremely useful to investigate in order to assess codes' capability to solve some of the individual physics and multiphysics problems at much reduced computational cost, even though it does not fully represent the behavior of the full-core.

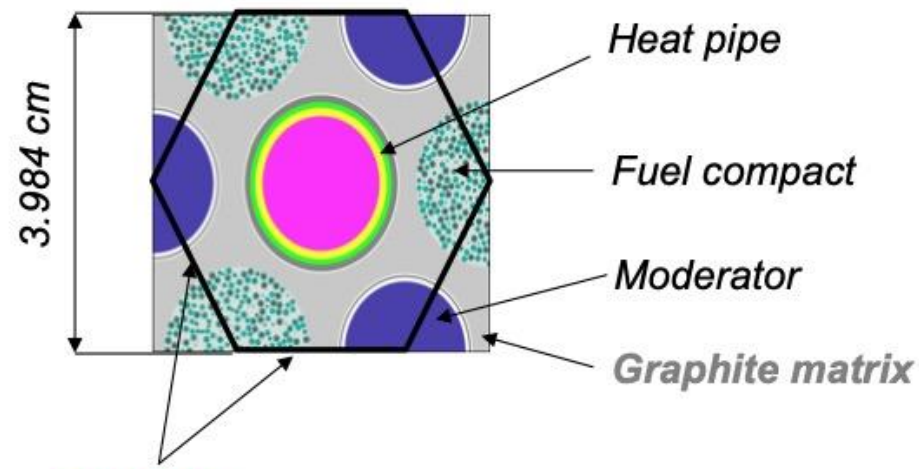

Refl. BC

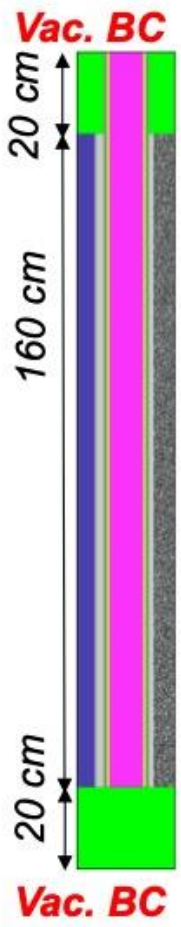

Figure 2-4. Axial/radial layout of unit-cell assembly. 


\section{Method Overview}

A combination of NEAMS tools was used to perform single-physics and coupled simulations on the modeling problems based on the micro-reactor design described in the previous section. All codes were developed within the MOOSE (Multiphysics Object-Oriented Simulation Environment) [12] framework. This section provides a brief overview of the codes capabilities, especially focusing on those employed in this project.

\subsection{Griffin}

Griffin is a reactor physics code which includes multiple functions to analyze the nuclear reactor core at steady-state or transient state $[6,15,16]$. It is built upon the MOOSE FEM framework and has a lot of common features with other MOOSE-based codes including mesh representation, FEM shape functions, parallel computing as well as the common pre-conditioned Jacobian-free NewtonKrylov executioner for solving the partial differential equations. Multiple schemes depending on the different treatment on the angular variable and different spatial discretization method are available to solve the radiation transport equation providing different fidelity to the solution. For instance, the SAAF-CFEM-SN is the scheme solving the self-adjoint angular flux formulation of the transport equation on a mesh with the continuous finite element method using the discrete ordinates method. CFEM-Diffusion is the scheme solving the radiation diffusion equation on a mesh with the continuous finite element method and DFEM-SN is the scheme using the $\mathrm{S}_{\mathrm{N}}$ angular discretization and solves the transport equations on a mesh with the discontinuous finite element method. In this project, all these transport schemes were tested on a 3-D unit-cell model for assessing their performance in our specific modeling problem. In Griffin, two equivalence methods the Super Homogenization (SPH) method and the Discontinuity Factors (DF) method are available to improve reactor physics calculations. The SPH method was used to improve the diffusion accuracy in calculating the reactor physics and was tested in the 3-D unit-cell model. Its accuracy and performance was also assessed with this unit-cell model.

The Griffin code is especially useful for multiphysics applications and includes functionalities to streamline their process. It can be imported as a MOOSE module and coupled with other physics codes [6]. For multiphysics simulations, Griffin is able to model the varying material properties by interpolating pre-generated look-up tables of the material properties using piecewise linear functions. In this project, two bidimensional look-up tables were prepared to model reactivity feedback effects in the multiphysics simulations. The first look-up table includes multigroup cross sections at different fuel and moderator temperatures to address temperature feedback effects from both fuel and moderator. The second look-up table includes multigroup cross sections at different fuel temperatures and different hydrogen contents in the YHx moderator. The goal is to model the reactivity feedback effects from the fuel temperature as well as from the hydrogen dissociation and redistribution process in the moderator. Verification studies were performed to test the accuracy of the interpolation method in Griffin using both bidimensional look-up tables.

\subsection{BISON}

BISON [18] is a finite element-based nuclear fuel performance code. BISON is capable to model a great variety of fuel systems (fuel and cladding), including traditional light water reactor fuels, accident tolerant fuels, metallic fuels and TRISO fuels. As a multidimensional multiphysics finite element code, BISON solves the fully-coupled nonlinear partial differential equations for thermomechanical problems in different dimensions, including full 3D, 2D-RZ axisymmetric, 
layered axisymmetric 1D, and spherically symmetric 1D systems. BISON fuel models are developed to describe temperature and burnup dependent thermal properties, fission product swelling, densification, thermal and irradiation creep, fracture, fission gas production and release. Recent accomplishments of BISON modeling can be found in ref [19].

In this report, two types of demonstrative simulations in different scales (engineering scale in millimeter vs. microscale in micrometer) using BISON code/models were performed. The first type of simulation is a microreactor full-core heat conduction and thermo-mechanical analysis. This simulation was mainly performed using the heat conduction module and tensor mechanics module in MOOSE, with supports of multiple BISON models. The second simulation is a standalone TRISO fuel particle performance analysis (2D axisymmetric (2D-RZ)). A 10-year fuel performance simulation for the TRISO fuel particle was performed to develop a final burnup of $\sim 1.67 \%$ FIMA $(\sim 17 \mathrm{MWd} / \mathrm{kgHN})$.

Beyond the single physics simulation, BISON is used as a sub-app in the multi-physics simulations coupled with Griffin, Sockeye, and SAM based on the MOOSE MultiApp system. Detailed discussion of the multi-physics can be found in section 7 .

\subsection{Sockeye}

Sockeye $[8,9]$ models heat pipe performance under a range of operating conditions. Development efforts are focused on heat pipe designs that are being proposed by industry for cooling microreactors, especially those cooled by liquid metals and employing annular screens or porous wick structures. The code includes features that provide valuable insights while designing a heat pipe, such as the capability to compute the operating limits-capillary limit, viscous limit, entrainment limit - for a particular heat pipe model. Sockeye originally employed a onedimensional, three-phase model for coolant thermal-hydraulics that is adapted from RELAP-7 [23]. To model startup operations, a three-phase model has recently been implemented that treats coolant melting. An alternative approach, the effective conductivity model, predicts the transient temperature profile in the heat pipe by solving a $2-\mathrm{D}, \mathrm{R}-\mathrm{Z}$ solid conduction problem where the coolant vapor core is assigned a very high thermal conductivity (e.g., $10^{5}$ to $10^{6} \mathrm{~W} / \mathrm{m}-\mathrm{K}$ ) to represent the heat transfer enhancement due to phase change. It is used to model the operating limits at each time step and to limit the heat removal rate if the heat pipe has failed, as demonstrated in Section 7.4.

For the work described in this report, the capability to evaluate analytic limits was used to ensure that the heat pipe design is reasonable. The effective conductivity model is used for all multiphysics calculations in this report. Single-physics, steady-state Sockeye simulations with the two-phase thermal-hydraulics model were used to determine the value of the thermal conductivity of the vapor core to use in the effective conductivity model. The newer three-phase model has not yet been used by the analysis in the report, but will need to be used in any future work for startup transients modeling.

\subsection{SAM}

The System Analysis Module (SAM) is a modern system analysis tool being developed at the Argonne National Laboratory for advanced non-LWR safety analysis [10]. It aims to provide fastrunning, whole-plant transient analyses capability with improved-fidelity for various advanced reactor types including liquid-metal-cooled, molten-salt cooled and fueled, gas-cooled, and heatpipe-cooled reactors. SAM takes advantage of advances in physical modeling, numerical methods, 
and software engineering, to enhance its user experience and usability. It utilizes an object-oriented application framework (MOOSE), and its underlying meshing and finite-element library (libMesh) and linear and non-linear solvers (PETSc), to leverage the modern advanced software environments and numerical methods.

In this report, SAM is utilized to model a Reactor Cavity Cooling System (RCCS), which removes heat from the reactor vessel during accident scenarios. In the test simulation used to demonstrate the capabilities of SAM, the heat conduction in the core is modeled through MOOSE and the heat transfer between the vessel and the RCCS is modeled in SAM. The SAM RCCS model consists of a two-dimensional solid structure representing the RCCS wall and a single one-dimensional fluid component which represents the air flow in the RCCS design. 


\section{Neutronics Analysis of Microreactor Unit-Cell Model}

The Griffin code was first used to perform neutronics simulation on the unit-cell model described in Section 2. With this simple geometry model, multiple verification studies were completed to demonstrate the Griffin code's capabilities in modeling the reactor physics of this micro-reactor in the multiphysics simulations. Some verification exercises are summarized in this section and an extended description is available in Appendix 1 and 2. Based on this analysis, a Griffin model which used the diffusion and SPH method for the 3-D unit cell model and a Griffin full core model using only diffusion were set up to enable the multiphysics simulations detailed in Section 7.

\subsection{Model description}

The reactor physics model for the HP-MR was created for the 3-D unit-cell model described in Section 2. An unstructured mesh created from Cubit [20] was used to model the 3-D unit-cell. The mesh elements are grouped into different blocks based on the material compositions in radial directions. In axial direction, the mesh size is $5 \mathrm{~cm}$ and the unit-cell is divided into 40 axial layers. The total volume for each material block was preserved in generating the mesh..

The multigroup cross sections used in the multiphysics simulations were generated from a separate sets of Serpent-2 Monte Carlo (MC) models [21]. These models have the same geometrical configuration with seven material zones, each representing the TRISO-fuel pins, the $\mathrm{YH}_{2}$ moderator pins, the graphite monolith, the heat pipe, the top/bottom beryllium reflectors, the heat pipe shell and the moderator shells. The TRISO-fuel particles were modeled with the fuel centroids realized from one random sampling. The heat pipe was modeled in Griffin and Serpent-2 by smearing its vapor, liquid and wick regions. The heat pipe shell and the moderator shells were also modeled by smearing the helium gaps and the surrounding steel envelopes. The multigroup cross sections were tallied on an 11-g energy structure with its upper energy boundary shown in Table 4-1.

Table 4-1. The upper energy boundaries $(\mathrm{MeV})$ for the 11-group neutron energy structure for Griffin reactor physics calculation.

\begin{tabular}{|c|c|c|c|c|c|}
\hline 1 & $8.0 \mathrm{E}-8$ & 2 & $1.8 \mathrm{E}-7$ & 3 & $6.25 \mathrm{E}-7$ \\
\hline 4 & $1.36 \mathrm{E}-6$ & 5 & $4.0 \mathrm{E}-6$ & 6 & $1.4873 \mathrm{E}-4$ \\
\hline 7 & $9.118 \mathrm{E}-3$ & 8 & $1.83 \mathrm{E}-1$ & 9 & $5.0 \mathrm{E}-1$ \\
\hline 10 & 1.353 & 11 & 15.0 & & \\
\hline
\end{tabular}

Multi-group cross section sets were generated with fuel and moderator temperatures varied among $600 \mathrm{~K}, 700 \mathrm{~K}, 800 \mathrm{~K}, 1000 \mathrm{~K}$, and $1200 \mathrm{~K}$ respectively. Other regions such as reflectors and heat pipes were assumed to have the same temperature as the moderator. In total $25 \mathrm{MC}$ simulations were performed to generate the multigroup cross section sets. The ISOXML utility code within Griffin was used to convert the multi-group cross section sets from the Serpent-2 output to the ISOXML format ready for Griffin to use [22].

The MC model used ENDF/B-7.1 cross section libraries directly at $600 \mathrm{~K}$, and $1200 \mathrm{~K}$ [24]. For temperatures at $700 \mathrm{~K}$ and $800 \mathrm{~K}$, the cross sections libraries at $600 \mathrm{~K}$ were used with the Serpent Doppler Broadening routine enabled. For temperatures at $1000 \mathrm{~K}$, the cross section libraries at 900 $\mathrm{K}$ were used with the Serpent Doppler Broadening routine enabled. The five temperature points were selected so that the $S(\alpha, \beta)$ libraries exist in the current ENDF/B-7.1 library at all these temperature points with no additional approximations. 
Each MC criticality simulation ran 1.0E9 particles, and the estimated statistical errors of the calculated k-effs are all around $4 \mathrm{pcm}$. As shown in Figure 4-1, the MC calculations showed that the k-eff of the 3-D unit cell decreases with increasing the fuel temperature which means the reactivity feedback from the fuel temperature is negative. However, the k-eff increases with increasing the moderator temperatures which means positive reactivity feedback from the moderator temperature (which does not account for thermal expansion and changes in density). Table 4-2 lists the calculated k-effs of those isothermal MC models where the temperatures are the same everywhere within the 3-D unit cell. It shows that the overall reactivity feedback will be negative when the temperatures within the fuel and moderator region are close and vary together during a transient.

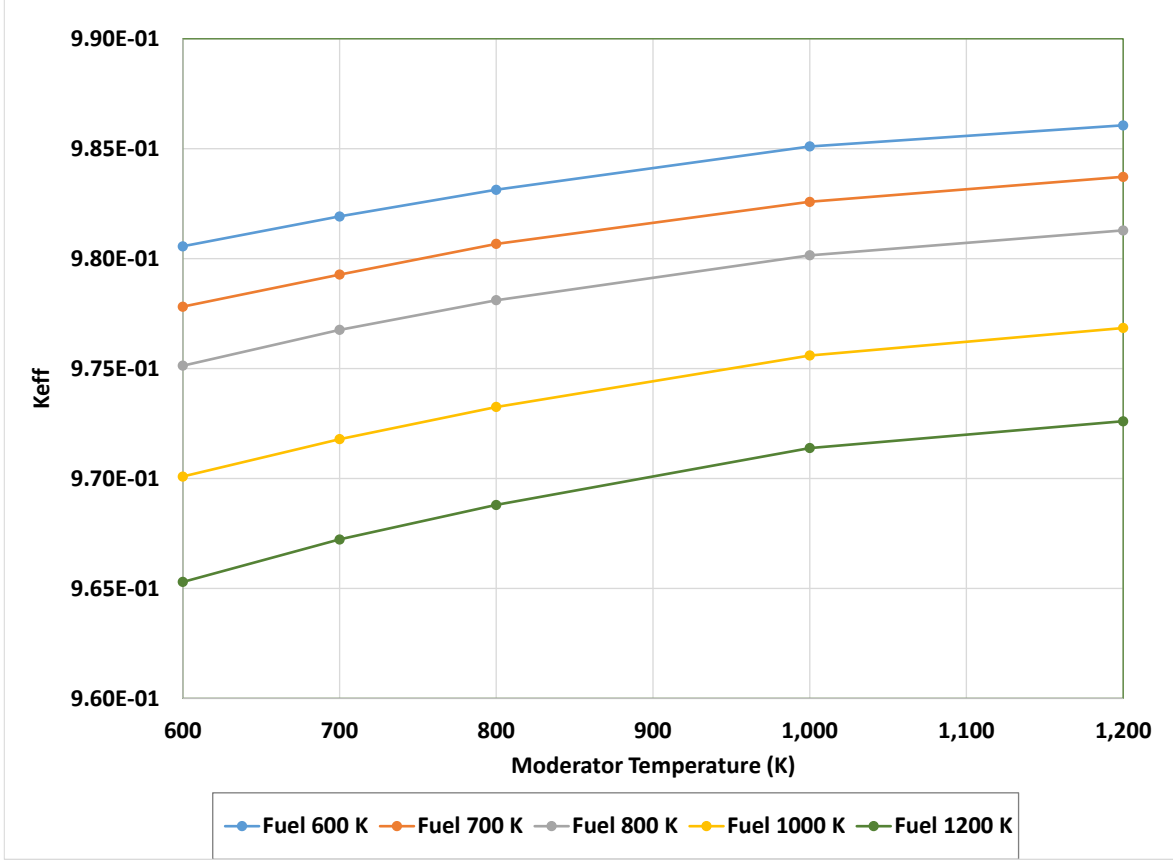

Figure 4-1. Serpent-2 calculated reference k-effs of the 3-D unit cell with its fuel and moderators at different temperatures.

Table 4-2. Serpent-2 calculated reference k-effs of the 3-D unit cell isothermal models.

\begin{tabular}{|l|l|l|l|l|}
\hline $\mathrm{T}=600 \mathrm{~K}$ & $\mathrm{~T}=700 \mathrm{~K}$ & $\mathrm{~T}=800 \mathrm{~K}$ & $\mathrm{~T}=1000 \mathrm{~K}$ & $\mathrm{~T}=1200 \mathrm{~K}$ \\
\hline $0.98056 \pm 4 \mathrm{pcm}$ & $0.97927 \pm 4 \mathrm{pcm}$ & $0.97811 \pm 4 \mathrm{pcm}$ & $0.97560 \pm 4 \mathrm{pcm}$ & $0.97260 \pm 4 \mathrm{pcm}$ \\
\hline
\end{tabular}

\subsection{Verification exercises of Griffin}

The Serpent-2 model of the 3-D unit-cell was setup not only to generate the multi-energy group macroscopic cross sections for Griffin, but also to provide reference k-eigenvalues and power distributions to verify the results obtained from Griffin. It also provides detailed fluxes solutions which will be used later in generating the SPH factors for the Griffin diffusion and SPH method. 


\subsubsection{Comparison of Serpent-2 solutions with various Griffin solvers}

Different schemes in Griffin such as diffusion, SAAF-CFEM-SN, the DFEM-SN have been applied to calculate the k-effs, the fluxes and power distributions for the 3-D heterogeneous unit-cell model at the selected temperature points. For $S_{N}$ methods, the maximum order of scattering anisotropy "NA" was set to 2 in the Griffin input and the Gauss-Chebyshev quadrature with different polar and azimuthal angles was used. Particularly, in this document, $S_{N}(1,3)$ represents there are $1 \times 3 \times$ $8=24$ directions in the $S_{N}$ calculation, and $S_{N}(3,3)$ represents a total number of 72 directions.

Table 4-3 lists the calculated k-effs from Griffin simulations for each isothermal unit-cell model and the deviation of the k-effs from the Serpent-2 reference results, as well as the amount of CPUhours Griffin spent to complete the simulation. The calculation time in Table 4-3 is just provided as indication of the relative computational cost for different solvers, while further model optimization (in terms of mesh and solver options) could help reduce computational costs. The simulations were all completed on the Argonne NSE division Linux workstation using 60 CPUs.

Table 4-3. Griffin calculated k-effs of the 3-D unit cell isothermal models using Diffusion, the SAAF-CFEM-SN and DFEM-SN schemes. $\triangle \mathrm{k}$-eff is the difference between Griffin and Serpent2 solution.

\begin{tabular}{|c|c|c|c|c|c|c|}
\hline \multicolumn{2}{|c|}{ Griffin Model } & $\mathrm{T}=600 \mathrm{~K}$ & $\mathrm{~T}=700 \mathrm{~K}$ & $\mathrm{~T}=800 \mathrm{~K}$ & $\mathrm{~T}=1000 \mathrm{~K}$ & $\mathrm{~T}=1200 \mathrm{~K}$ \\
\hline \multirow{3}{*}{ Diffusion } & k-eff & 0.97603 & 0.97306 & 0.97052 & 0.96560 & 0.96068 \\
\hline & $\Delta \mathrm{k}$-eff & -453 & -621 & -759 & -999 & -1192 \\
\hline & $\begin{array}{l}\text { CPU- } \\
\text { hour }\end{array}$ & 2.81 & 2.78 & 2.94 & 2.87 & 2.82 \\
\hline \multirow{3}{*}{$\begin{array}{c}\text { SAAF- } \\
\text { CFEM-SN } \\
\quad(1,3)\end{array}$} & k-eff & 0.98976 & 0.98783 & 0.98639 & 0.98312 & 0.97952 \\
\hline & $\Delta \mathrm{k}$-eff & 920 & 966 & 828 & 752 & 692 \\
\hline & $\begin{array}{l}\text { CPU- } \\
\text { hour }\end{array}$ & 36.33 & 36.14 & 37.26 & 37.26 & 37.82 \\
\hline \multirow{3}{*}{$\begin{array}{c}\text { SAAF- } \\
\text { CFEM-SN } \\
\quad(3,3)\end{array}$} & k-eff & 0.98506 & 0.98351 & 0.98221 & 0.97931 & 0.97601 \\
\hline & $\Delta \mathrm{k}$-eff & 450 & 424 & 410 & 371 & 340 \\
\hline & $\begin{array}{l}\text { CPU- } \\
\text { hour }\end{array}$ & 116.84 & 116.44 & 119.61 & 120.91 & 118.56 \\
\hline \multirow{3}{*}{$\begin{array}{c}\text { DFEM-SN } \\
(1,3)\end{array}$} & k-eff & 0.98883 & 0.98707 & 0.98560 & 0.98242 & 0.97891 \\
\hline & $\Delta \mathrm{k}$-eff & 827 & 780 & 749 & 683 & 630 \\
\hline & $\begin{array}{l}\text { CPU- } \\
\text { hour }\end{array}$ & 224.39 & 227.59 & 227.64 & 229.89 & 228.43 \\
\hline
\end{tabular}

Table 4-3 shows that the Griffin model using the diffusion scheme is the fastest. With 60 CPUs, each simulation was completed within three minutes. However, the calculated k-effs have large errors compared with the MC reference results. $\Delta \mathrm{k}$-eff is about $450 \mathrm{pcm}$ at $600 \mathrm{~K}$ and grows bigger to about $1200 \mathrm{pcm}$ at $1200 \mathrm{~K}$. We used the k-effs at the neighboring temperature points to calculate reactivity temperature feedback coefficients $\alpha$. For instance, at $600 \mathrm{~K}$, Figure 4-2 showed that using the diffusion scheme has overestimated the value of $\alpha$ by more than $100 \%$ at most of the temperature points. In addition, Figure 4-3 shows that diffusion has completely miscalculated the axial power distribution in this 3-D unit-cell model indicating that any results from the diffusion calculation is not reliable, which is not surprising due to the high level of heterogeneity in this model. 
The k-effs, the reactivity coefficients $(\alpha)$ as well as the axial power distributions calculated from the three $S_{N}$ schemes have also been compared with the Serpent- 2 reference results. The $S_{N}$ schemes are much more accurate than the diffusion scheme. There are still about $400 \mathrm{pcm}$ differences in the calculated k-effs in the $\mathrm{S}_{\mathrm{N}}(3,3)$ calculations, which can be improved with higher $\mathrm{S}_{\mathrm{N}}$ orders or with more material zones used in the 3-D unit cell model (details can be shown in Appendix 2). The reactivity coefficients and the axial power distributions calculated from the $S_{N}$ methods are all matching very well with the reference results. However, as shown in Table 4-3, all of the $\mathrm{S}_{\mathrm{N}}$ calculations are slow.

The long computer running time makes the $S_{\mathrm{N}}$ scheme impossible to be applied in our multiphysics simulations at this moment. In Griffin, different acceleration schemes are under development to accelerate the $\mathrm{S}_{\mathrm{N}}$ calculation. At the current stage, none of these acceleration schemes were compatible in the MultiApp framework, so were excluded from our verification work. To conclude, the Diffusion solver is fast and suitable for multiphysics, but does not provide reliable results on this heterogeneous model. The next section discusses the use of SPH correction factors to improve accuracy of the Diffusion solver.

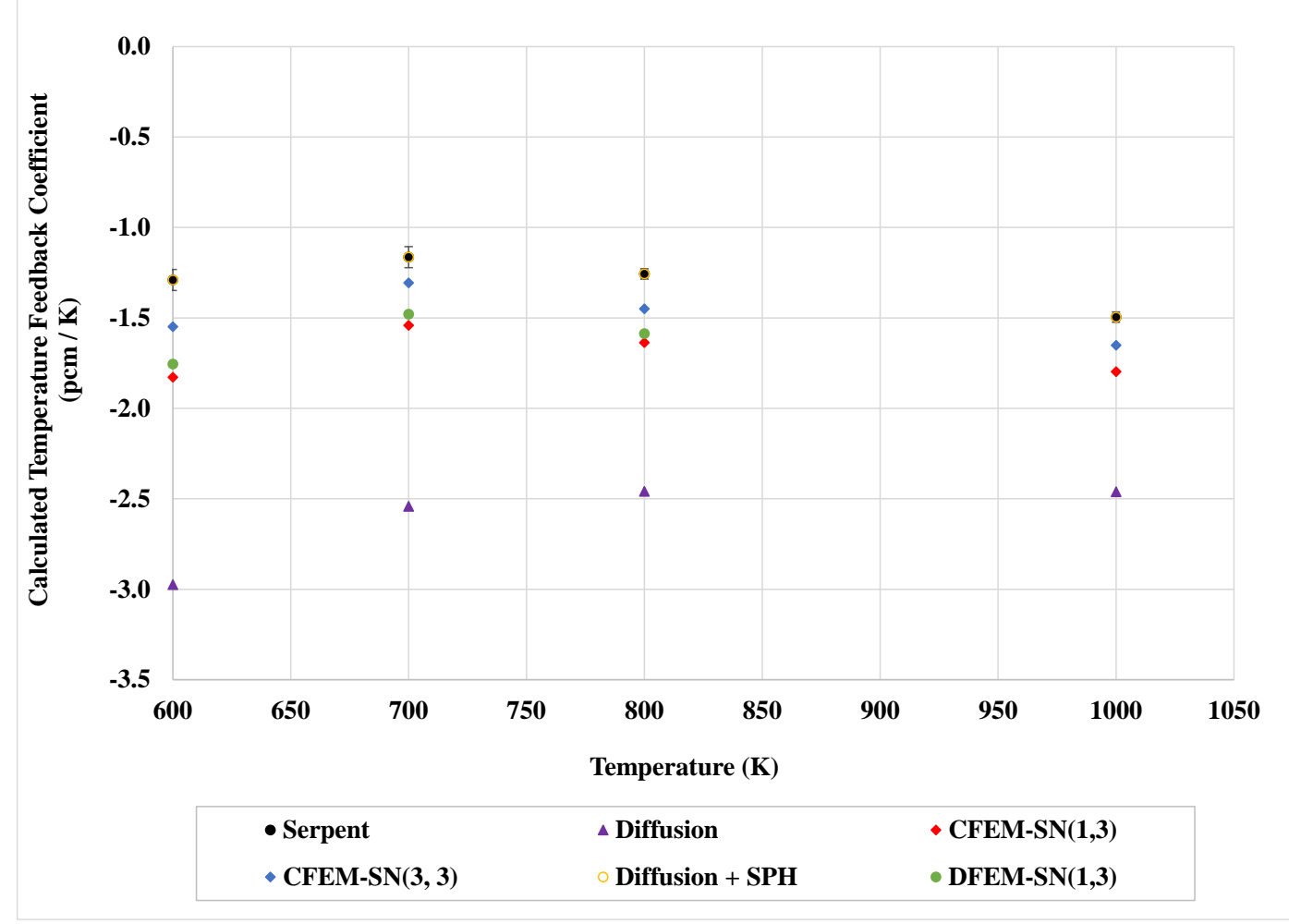

Figure 4-2. Calculated reactivity temperature feedback coefficients for the 3-D unit-cell isothermal models. 


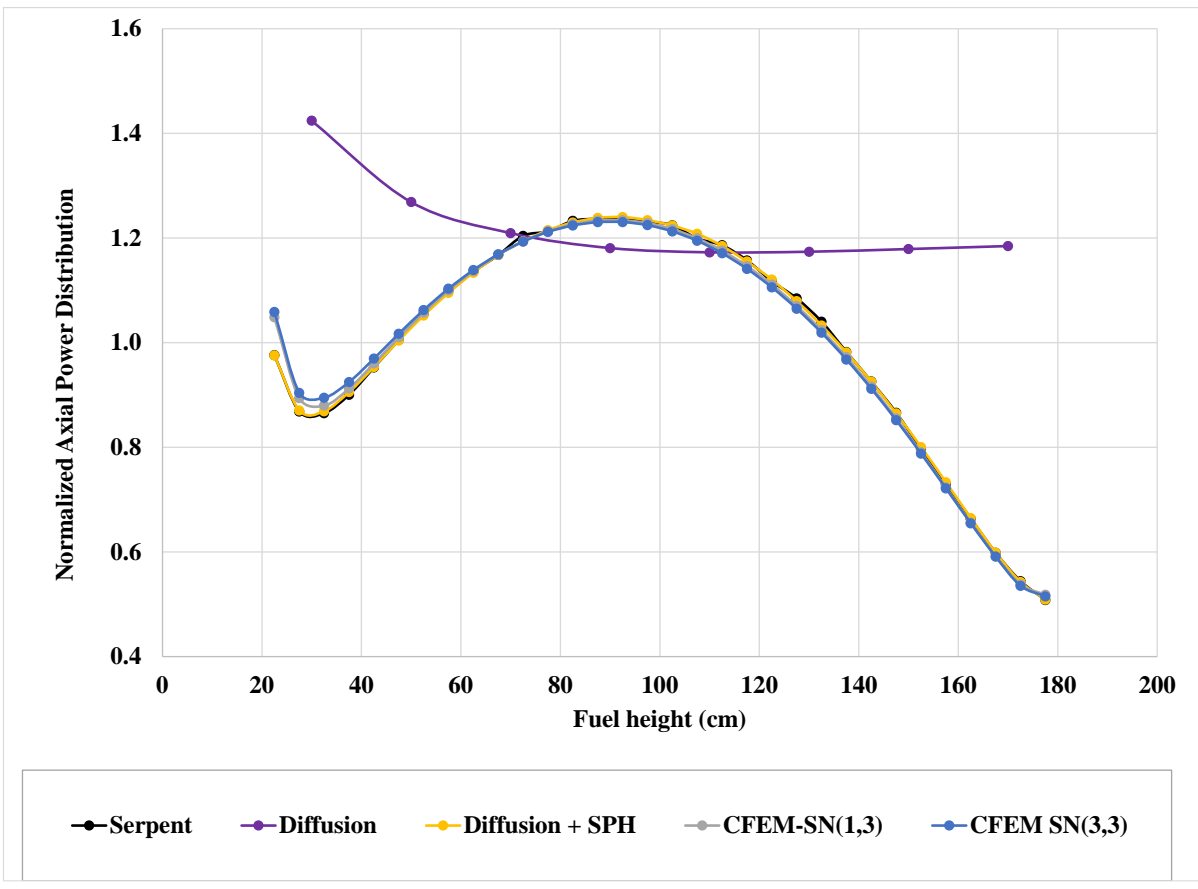

Figure 4-3. Calculated axial power distributions for the 3-D unit-cell isothermal model at $800 \mathrm{~K}$.

\subsubsection{Detailed verification of the SPH solution}

Griffin has two equivalent methods, the Discontinuity Factors (DF) and the Super Homogenization $(\mathrm{SPH})$, that can be used to reduce the errors stemming from geometrical homogenization by preserving region-averaged reference quantities such as fluxes, currents, or reaction rates obtained from a heterogeneous model [25]. Both of them are correcting the cross sections in solving the neutron transport equation. Previously, the diffusion and SPH method has been applied to improve the accuracy of the diffusion calculation on a full core homogenized reactor model [3].

Both the 3-D unit cell and the full-core neutronics models of this microreactor have its main components (e.g., fuel pins, moderator pins, etc.) represented as separate regions. In this study, we have applied the diffusion and SPH method to the 3-D unit cell model. The main purpose of this section is to confirm that this method can improve the accuracy of the diffusion calculation in the multiphysics simulations which are based on a heterogeneous core model. The reference quantities which are preserved are the fluxes from the Serpent-2 simulations. The procedure to generate the $\mathrm{SPH}$ factors is the following:

1. An additional equivalence zone map first needs to be described in the Griffin input. The equivalence zones are regions where we decide to preserve the reference region-averaged reaction rates. For our 3-D unit cell model, every material block in the finite element mesh was an independent equivalence zone. Particularly, in the axial direction, equivalence zones were assigned for every $5 \mathrm{~cm}$ axial layer. In total, 208 equivalence zones were setup for the 3-D unit cell model. Using fewer number of equivalence zones in the axial directions was also tested. The approach adopted for generating this equivalence zone map is discussed in more detail in Appendix 1.

2. The physical quantities to be preserved in the SPH method are the reaction rates. Given Griffin model used the cross sections being directly generated from the MC simulations, 
the region-averaged multigroup neutron fluxes were an input to Griffin in each equivalence zone. They were tallied using the Serpent- 2 model at each temperature point and were converted to the ISOXML format by a user utility code.

3. Griffin used these reference fluxes to compute SPH factors. Particularly, the SPH factor $\mu_{m, g}$ is computed such that $\Sigma_{m, g}^{M C} \phi_{m, g}^{M C}=\mu_{m, g} \Sigma_{m, g}^{M C} \phi_{m, g}$, where " $\mathrm{m}$ " is the indices for the equivalence zone and " $\mathrm{g}$ " is the indices for the energy group, $\Sigma_{m, g}^{M C}$ is the macroscopic cross section generated from the MC simulation at zone "m" and energy group " $\mathrm{g}$ ", $\phi_{m, g}^{M C}$ is the reference flux and $\phi_{m, g}$ is the flux calculated with the $\mu_{m, g}$ introduced into the diffusion equation. This modified diffusion equation is non-linear and is solved using the Preconditioned Jacobian-Free Newton Krylov (PJFNK) method in Griffin.

The reference k-eff is an import input parameter to guarantee that the diffusion and SPH method is used correctly. If the SPH factors are calculated correctly, the k-eff and the zone-averaged fluxes obtained from the modified diffusion equation will match the reference values as shown in Figure 4-2 and Figure 4-3 for our 3-D unit cell models.

In multiphysics simulations, the SPH factors at other temperatures were interpolated among calculated SPH factors at discrete temperature points. Detailed discussions on the interpolation scheme of the cross sections and SPH factors in Griffin are included in the next section. A user utility code was used to convert the SPH factors computed from the iterative process at each equivalence region, energy group as well as at each temperature point to a lookup table format.

Figure 4-4 shows the k-effs obtained from the Griffin diffusion calculation with cross sections and SPH factors interpolated among $800 \mathrm{~K}$ and $1000 \mathrm{~K}$. The results show that the k-effs at the interpolated temperature points are very close to the expected k-effs from a linear extrapolation of the MC reference value. The label " $1 \mathrm{~d}$ grid" and " $2 \mathrm{~d}$ grid" represents the different dimensions of the temperature grids used in the interpolation and will be discussed in the next section. More on the accuracy of using the interpolated cross sections and SPH factors for multiphysics simulations are also included in that section. In this study, the diffusion and SPH method has been used for multiphysics simulations of a load following transient in section 7.1. 


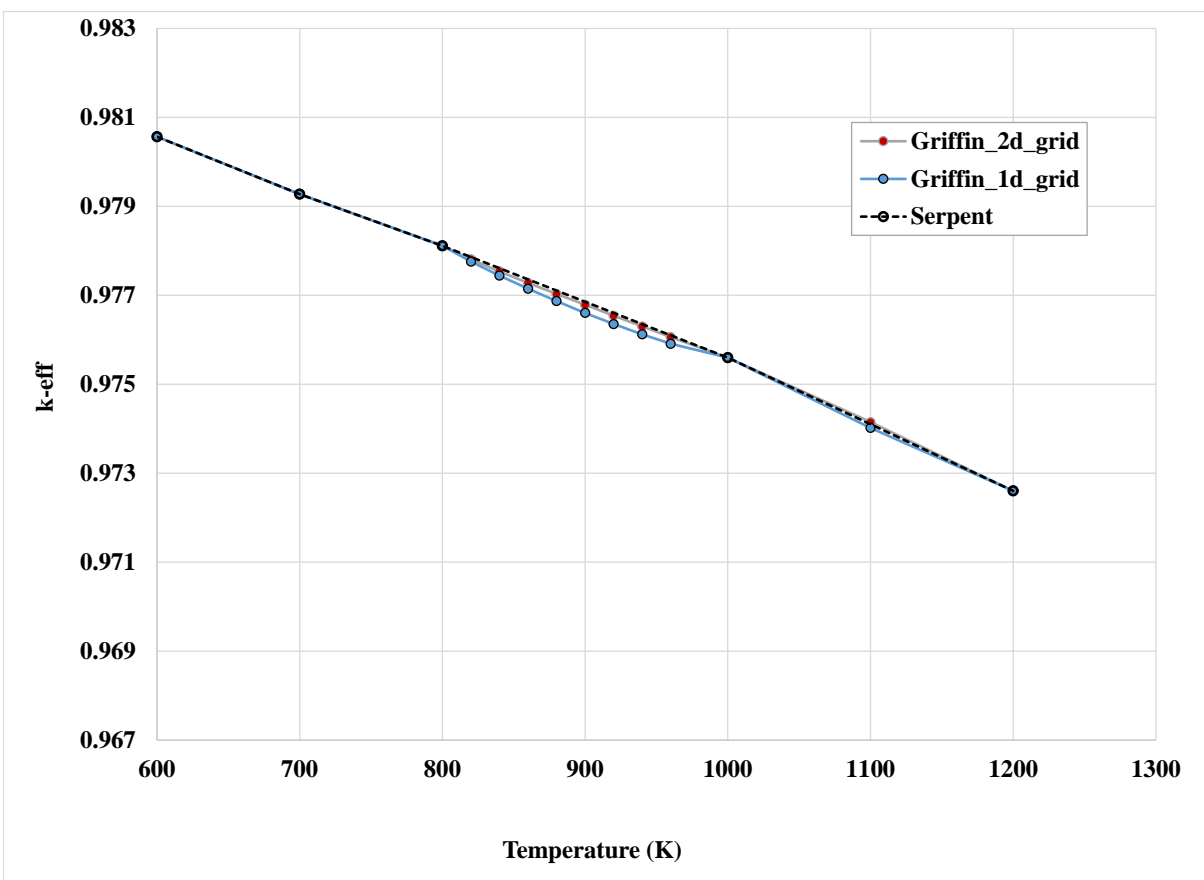

Figure 4-4. Calculated k-effs for the 3-D unit-cell isothermal model at different temperatures using the diffusion and SPH method.

\subsection{Generation and verification of bidimensional tabulated cross-sections for Griffin}

The material property dependence on a variable can be modeled in MOOSE using piecewise linear interpolations given the material property evaluated at the different variable locations. This variable is referred as a "grid" variable in the multiphysics simulation input, and a look-up table describing the property values at the grid points are usually prepared for multiphysics transient simulations. The objective of the work described in this section is to confirm Griffin's ability to interpolate cross-sections from bidimensional libraries using both the temperature and hydrogen contents as the dependent variables.

\subsubsection{Tabulation of fuel/non-fuel temperatures}

In our microreactor model, the material temperature grids were setup to allow Griffin obtaining temperature dependent multigroup cross sections. Five temperature points at $600 \mathrm{~K}, 700 \mathrm{~K}, 800 \mathrm{~K}$, $1000 \mathrm{~K}$, and $1200 \mathrm{~K}$ were used as grid points for generating the cross section look-up table.

The temperature gradient between the heat source (fuel pins) to the heat sink (heat pipes) is small in this microreactor. Particularly, at steady state within the 3-D unit cell model, the average moderator temperature is only about 3 degree $\mathrm{C}$ lower than the average fuel temperature. Therefore, this small difference was first ignored in the reactor physics analysis. A one-dimension temperature grid using the "fuel temperature" variable was setup for the multiphysics simulations. Multigroup cross sections were generated using the five isothermal Serpent- 2 models described at the beginning of section 4. This look-up table was referred as the "1d-grid" in Figure 4-4.

The temperature difference between the fuel region and the non-fuel region, as being demonstrated later in the load following transient simulations, increases while the reactor power increases. The 
reactivity temperature feedback effects from the moderator are positive for this 3-D unit-cell model. A two-dimension temperature grid which described the multigroup cross section dependence on both the fuel and the moderator temperatures was also setup for the multiphysics simulations. This look-up table was referred as the "2d-grid" in Figure 4-4.

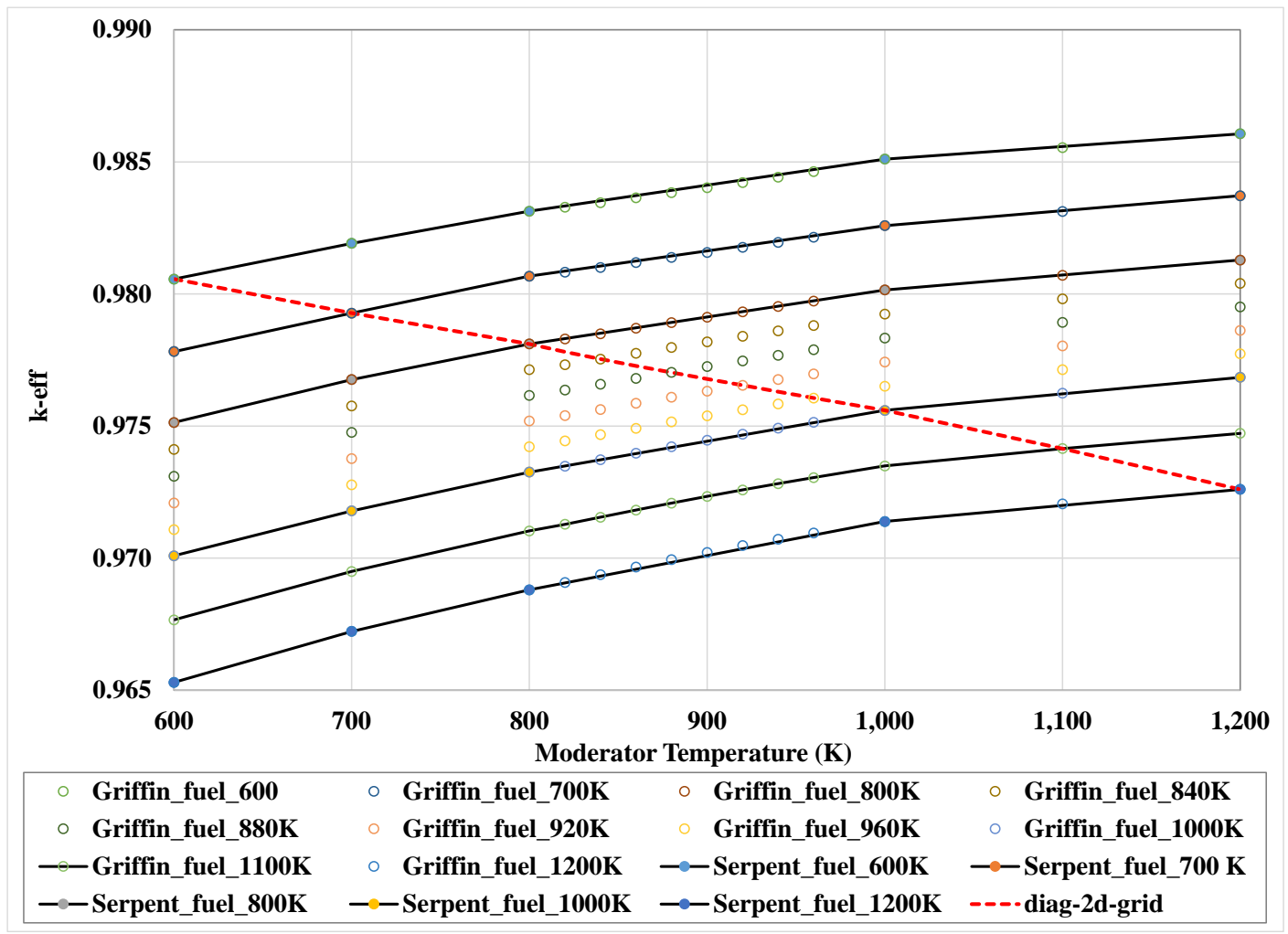

Figure 4-5. Calculated k-effs for the 3-D unit-cell at different temperatures using 2d-grid cross section table in the diffusion and SPH method compared with the Serpent-2 reference results.

Figure 4-5 is an expanded two-dimension comparison of Figure 4-4 at all the grid points of the $2 \mathrm{~d}$ look-up table. The "diag-2d-grid" line in the figure is exactly the same line as the line "Griffin_2d_grid" in Figure 4-4. At each interpolated point, the multigroup cross sections and the $\mathrm{SPH}$ factors are the linear combination of the four neighboring grid values. The Griffin reactor physics calculation of the k-effs used the diffusion and SPH method. The k-effs calculated from Griffin matched the reference results exactly at the grid point as expected. At those interpolated positions, the k-effs also followed the lines linearly extrapolated from the Serpent-2 reference results. For temperature dependence, the multiphysics transient simulations used both the 1d-grid and 2d-grid look-up tables and results are discussed in section 7.1.

\subsubsection{Tabulation of fuel temperature and $\mathrm{H}$-content}

The H-content in the YHx moderator may also vary during transients because hydrogen can dissociate and loss from the $\mathrm{YHx}$ matrix. With less neutron moderation from $\mathrm{H}$, the neutron spectrum becomes harder and k-eff is reduced. A H-content grid was setup to define the multigroup cross section dependence on its density in the moderator. Similarly, a two-dimension look-up table with one dimension for the material temperature change (fuel temperature) and the other dimension 
for the H-content change in the moderator was prepared for future multiphysics simulations. Griffin's ability to interpolate cross-section from such bidimensional library is demonstrated in this section prior to attempting the Griffin/SWIFT coupling planned in future work.

Previous Serpent-2 isothermal models at the five temperature grid points were modified to allow the $\mathrm{H}$ density varying from its reference atom density of $\mathrm{YHx}$ when $\mathrm{x}=2.0$ to $\mathrm{x}=1.0$. Within these range, nine additional grid points were selected to generate the 2-D look-up table. Figure 4-6 plotted the calculated k-effs at each of the 2-d grid points from the Serpent- 2 models. The statistical errors are all about $7 \mathrm{pcm}$. The reactivity feedback from the $\mathrm{H}$ density reduction is negative.

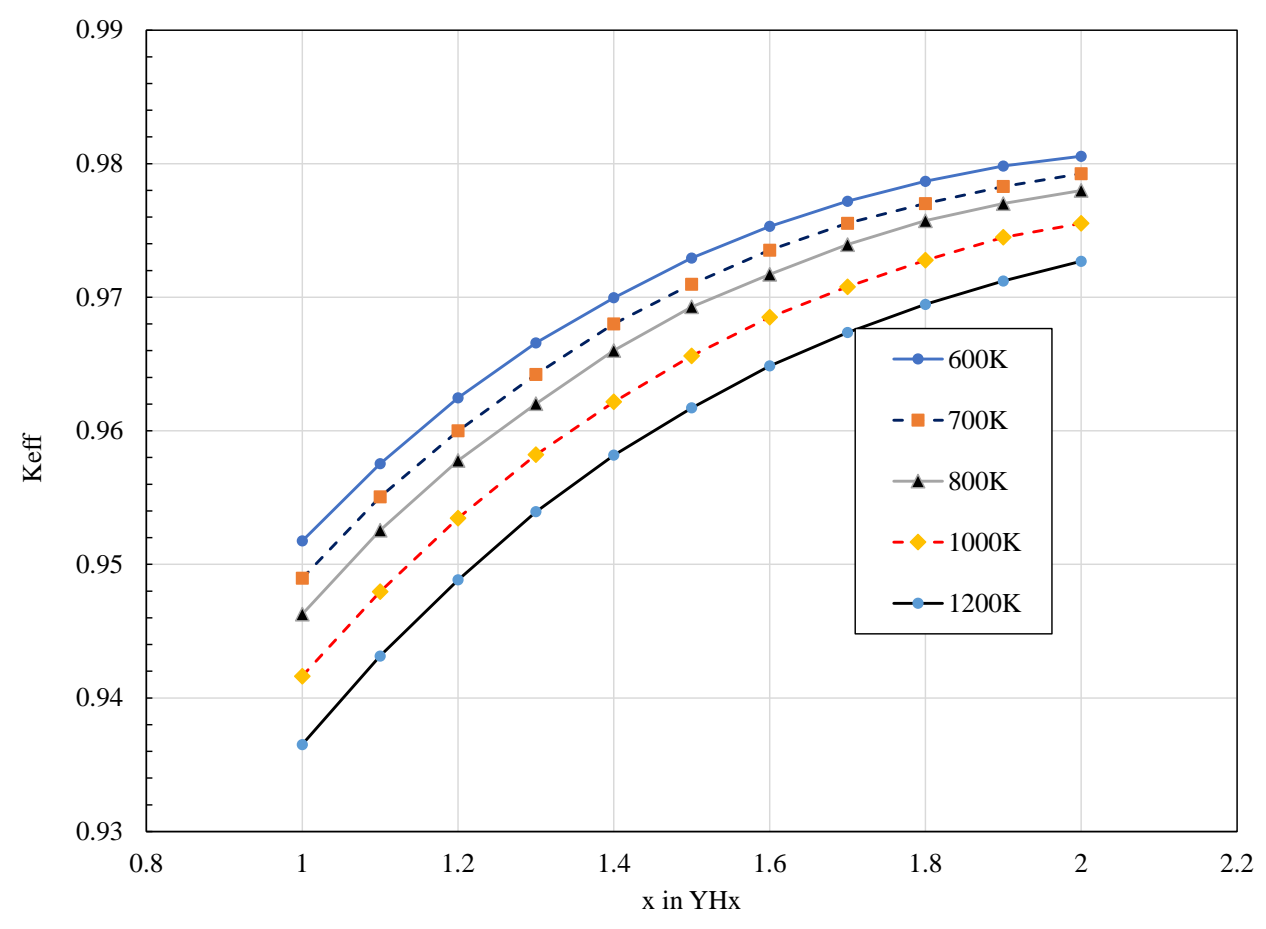

Figure 4-6. Calculated k-effs from Serpent-2 models at different temperatures and different hydrogen concentrations.

\subsubsection{Verification of the accuracy of the interpolation of bidimensional tabulated cross section}

A dedicated study has been performed to test the accuracy of using the multigroup cross sections interpolated in Griffin for reactor physics calculations. A fuel-temperature and H-content 2-D lookup table which only includes 18 grid points $(\mathrm{T}=600 \mathrm{~K}, 800 \mathrm{~K}$ and $1200 \mathrm{~K}$ and $\mathrm{x}=1.0,1.2,1.4,1.6$, 1.8 and 2.0) were setup using MC results. The cross sections for reactor physics calculations at additional grid points ( $\mathrm{T}=700 \mathrm{~K}$ and $1000 \mathrm{~K}$ and $\mathrm{x}$ at every 0.1 within $[1.0 ; 2.0])$ were interpolated with Griffin from this 2-D look-up table. Then, the Griffin SAAF-CEFM-SN(3,3) scheme was used to perform two sets of calculations. The first set was performed with the interpolated multigroup cross sections at $700 \mathrm{~K}$ and $1000 \mathrm{~K}$. For reference, the second set was performed using multigroup cross sections directly from Serpent-2 outputs at the same temperature and H-content.

Figure 4-7 a) shows that the calculated k-effs from the two sets of $S_{N}$ calculations match each other very well for all cases. The maximum difference of the k-effs are within $20 \mathrm{pcm}$ if the fuel temperature is at $700 \mathrm{~K}$ as shown in Figure $4-7 \mathrm{~b}$ ). The maximum difference is $63 \mathrm{pcm}$ if the fuel 
temperature is at $1000 \mathrm{~K}$. It is slightly larger due to the coarser temperature grid around $1000 \mathrm{~K}$. The fuel temperature reactivity feedback coefficients were also calculated by using the k-effs calculated from these two sets of $S_{N}$ calculations. The differences are within the range of $1 \%-7.5 \%$ with the hydrogen content $x$ varied at the 11 grid points. In addition, the axial power distributions were also compared and Figure 4-7 c) shows that the axial power distributions match extremely well for the case with the fuel temperature at $1000 \mathrm{~K}$ and $\mathrm{x}$ at 1.1 . The maximum relative differences of the axial power distributions for all cases are shown in Figure 4-7 d). The maximum differences are very small $<0.07 \%$, although the differences are slightly larger for those cases with the fuel temperature at $1000 \mathrm{~K}$ again due to the coarser temperature grid used in the interpolations. More details are discussed in Appendix 2.

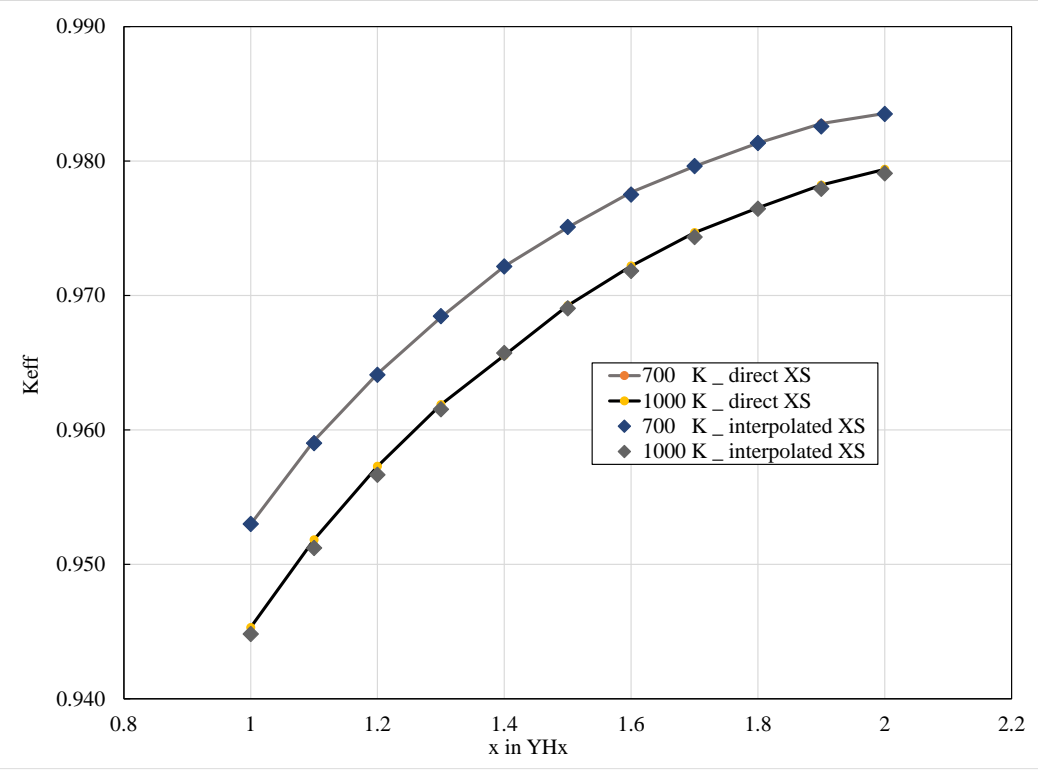

(a)

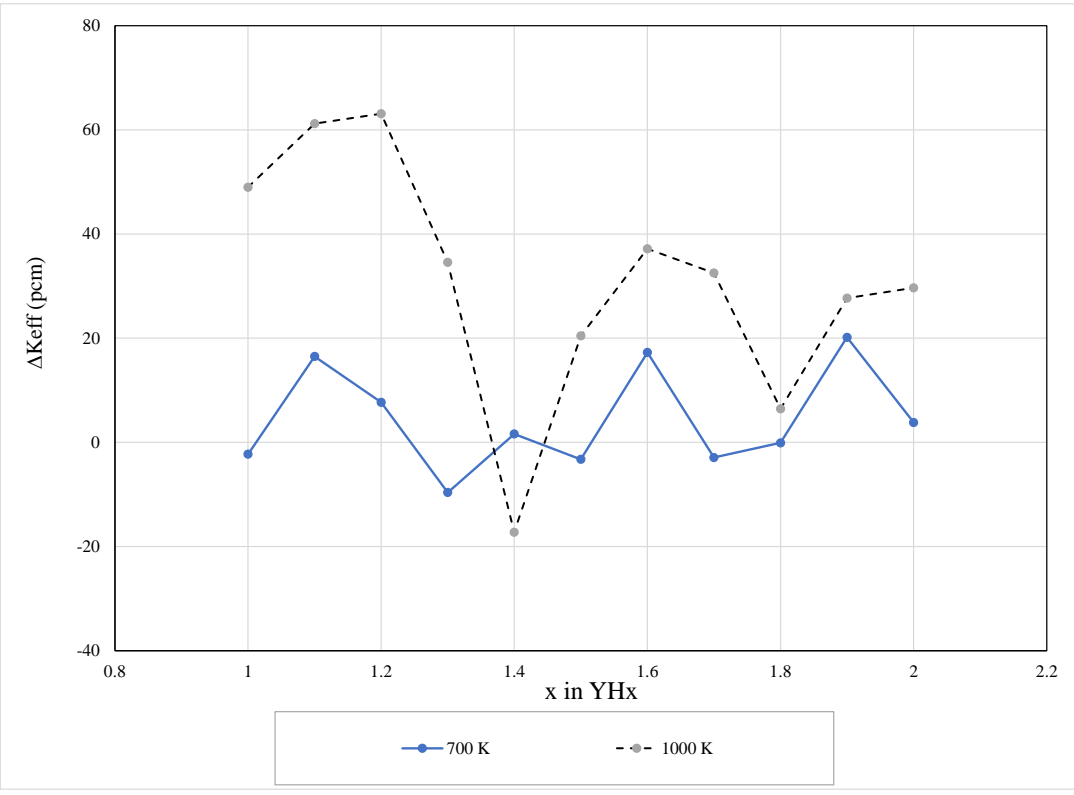

(b) 


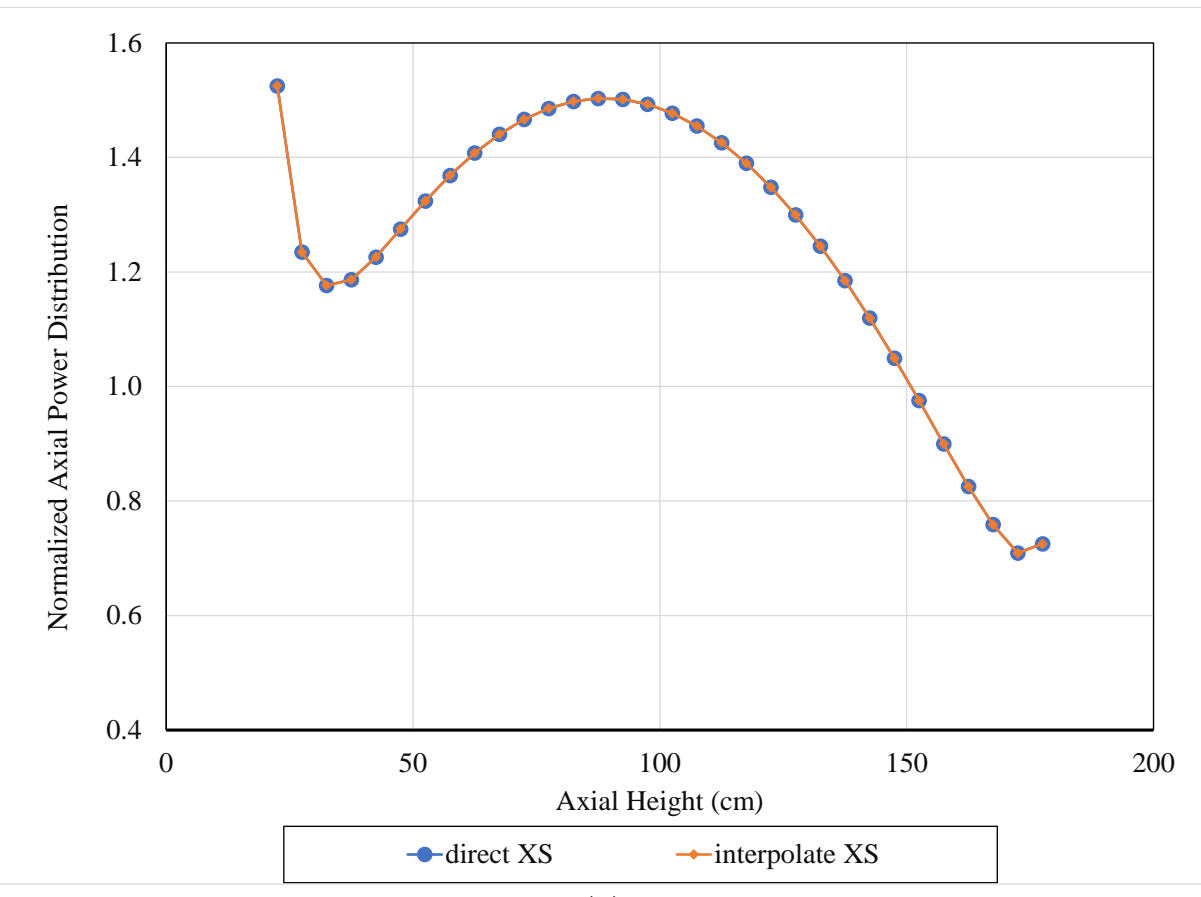

(c)

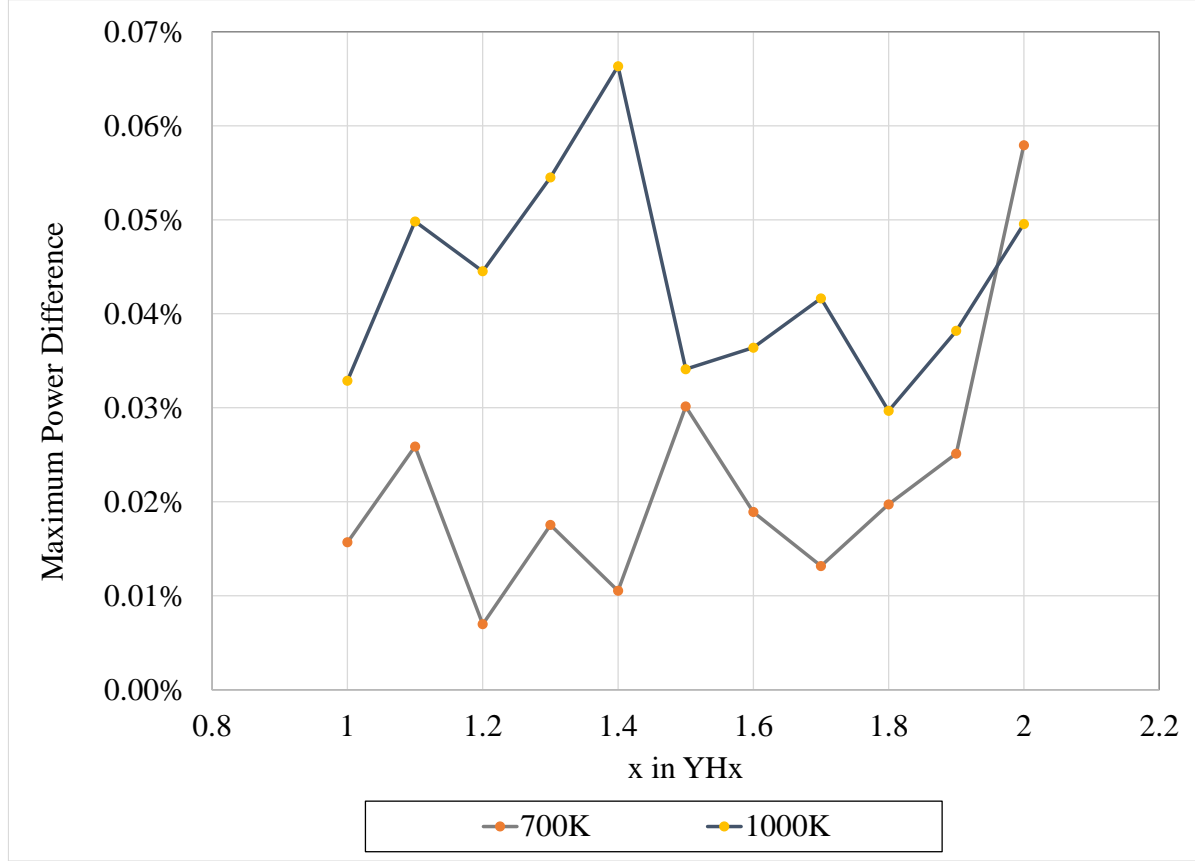

(d)

Figure 4-7. Comparison of the Griffin SAAF-CFEM-SN calculation with multigroup cross sections directly converted from Serpent-2 output or with multigroup cross sections interpolated from the 2-d look-up table a) k-effs for all $\mathrm{x}$ cases at $700 \mathrm{~K}$ and $1000 \mathrm{~K}$ b) deviation in K-eff for all these cases c) normalized axial distributions for the case with $T=1000 \mathrm{~K}$ and $\mathrm{x}=1.1 \mathrm{~d}$ ) maximum relative differences of axial power distributions for all cases. 
A similar verification study was also performed for studying the accuracy of using the interpolated multigroup cross sections and SPH factors in the diffusion and SPH method. First, a fuel and moderator temperature 2-D look-up table was created by only including grid values at fuel and moderator temperature $\mathrm{T}=600 \mathrm{~K}, 800 \mathrm{~K}$ and $1200 \mathrm{~K}$. The multigroup cross sections and the SPH factors for fuel temperatures at $700 \mathrm{~K}$ and $1000 \mathrm{~K}$ were interpolated from this 2-D look-up table. For moderator temperatures at $700 \mathrm{~K}$ and $1000 \mathrm{~K}$, the cross sections and SPH factors were interpolated among four grid values, other points are interpolated among two grid values. Then, two sets of diffusion calculation with SPH method were performed either using the interpolated cross sections and SPH factors, or using cross sections directly obtained from the Serpent-2 calculations and SPH factors directly calculated from Griffin PJFNK method. The differences of k-effs from the two sets of calculations are shown in Table 4-4. Overall, the differences are small with maximum differences less than $50 \mathrm{pcm}$. The differences are slightly larger at those points where four grid values were used for interpolation. The fuel temperature feedback coefficients using the two sets of k-effs with the direct cross sections or with the interpolated cross sections were also calculated and compared to each other with differences at the four moderator temperature points varying from $0.23 \%$ to $4.7 \%$. In addition, the axial power distribution profiles are found to be very similar from the two sets of calculations, with maximum differences within $5 \%$ everywhere. More details are discussed in Appendix 1.

Table 4-4. Difference in k-effs [pcm] with the direct or interpolated cross sections and SPH factors.

\begin{tabular}{|l|l|l|l|l|l|}
\hline Moderator T & $\mathrm{T}=600 \mathrm{~K}$ & $700 \mathrm{~K}$ & $800 \mathrm{~K}$ & $1000 \mathrm{~K}$ & $1200 \mathrm{~K}$ \\
\hline Fuel T=700 K & -0.5 & -1.2 & 6.6 & 48.9 & 4.9 \\
\hline Fuel $\mathrm{T}=1000 \mathrm{~K}$ & -2.3 & -20.8 & -18.1 & 16.0 & -9.0 \\
\hline
\end{tabular}

\subsection{Griffin reactor physics model with SWIFT-generated varying H-content}

With the fuel temperature and H-content 2-D look-up table prepared, a Griffin input was also setup to demonstrate that the Griffin code can be used to model the $\mathrm{H}$ dissociation and redistribution effects in the reactor physics calculations. In particular, the H-content " $\mathrm{x}$ " within the YHx moderator pins were calculated by SWIFT [11] based on a steady state core temperatures obtained from the multiphysics simulations. It varies between 1.93 to 1.98 within the 3-D unit cell model as shown in Figure 4-8 a). The calculation assumed operating temperature around $800 \mathrm{~K}$ at the condenser of the heat pipe.

To model the different $\mathrm{H}$-contents at different meshes with Griffin, one applied a user defined "AuxVariable" which reads the "x" from SWIFT output directly and specifies the value to each finite element mesh. The fuel and moderator temperatures were set to the average value at $852 \mathrm{~K}$. The Griffin $S_{N}(3,3)$ has been used to calculate the k-eff and its axial power distributions. An additional Griffin model which has the fuel and moderator temperature at the average value $852 \mathrm{~K}$ and " $\mathrm{x}=2.0$ " was also setup for comparison.

Overall, the k-eff of the 3 -D unit cell is calculated to be 0.98142 for the case with " $\mathrm{x}=2.0$ ". It is 0.98088 for the case with the Hydrogen x-distribution from SWIFT. A 54 pcm reduction on the keffs was observed due to the loss of neutron moderation and a harder neutron spectrum in the 3-D unit cell model. Figure 4-8 shows that the axial power distributions are very similar for these two cases with maximum differences smaller than $1.02 \%$. As suggested from SWIFT simulation, H- 
content has a dip at the bottom part of the moderator pin. Consequentially the neutron spectrum around this position is harder than the homogeneous case where " $\mathrm{x}=2.0$ " around the same position, and less power is produced around this position as shown in Figure 4-8 b).

To be noted, the average moderator temperature at normal operation for this HP-MR is low ( 800K) and the hydrogen dissociation and redistribution effects are expected to be small. However, when reactors are under the transient scenarios (as some of them shown in section 7), the moderator temperatures can be significantly larger. Then the neutronic impact coming from hydrogen dissociation effects might become more significant. This numerical study simply demonstrated that Griffin can be readily applied to model the varying $\mathrm{H}$-content in the multiphysics transient simulations.

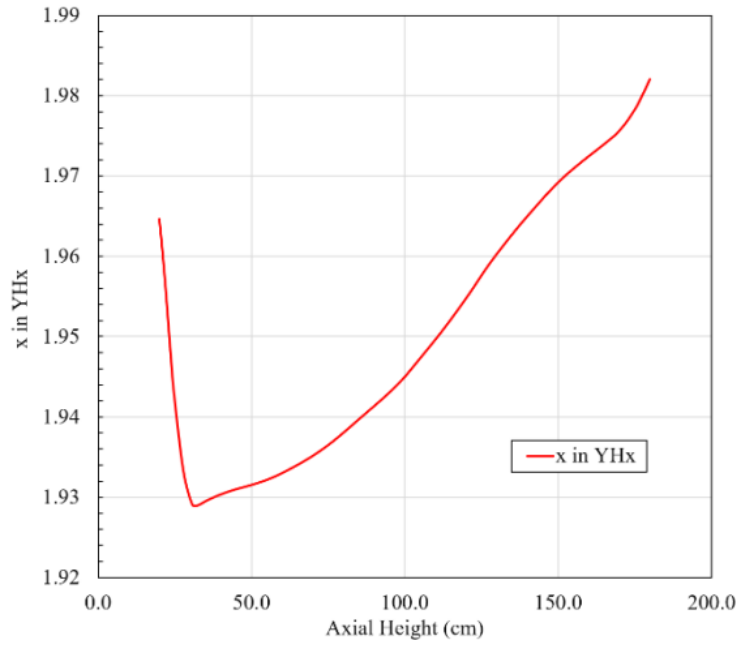

a)

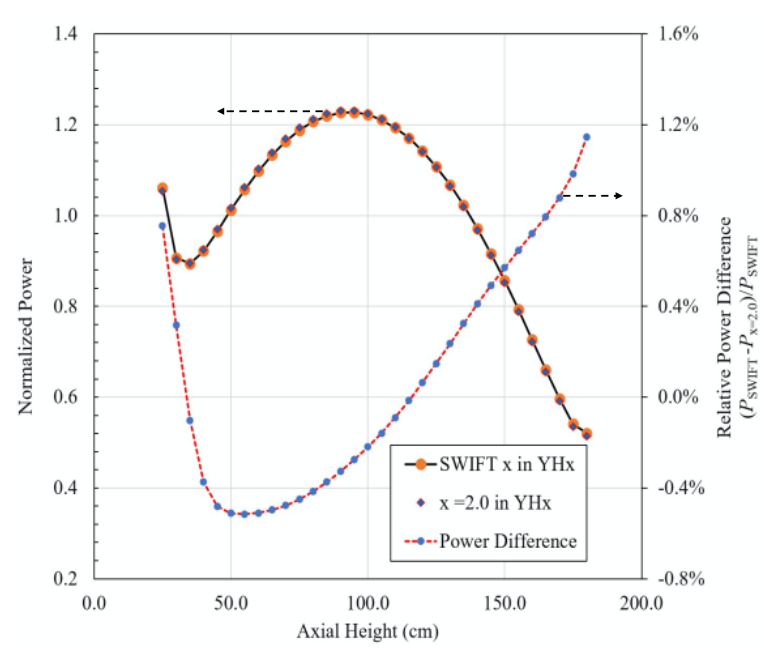

b)

Figure 4-8. a) Hydrogen content throughout the axial direction in the $\mathrm{YH}_{2}$ moderator pin as estimated by SWIFT, b) Griffin SN(3,3) calculated axial power distributions for the 3-D unit cell models with fuel temperature at $852 \mathrm{~K}$ and with $\mathrm{H}$-content in the YHx moderator: $\mathrm{x}=2.0$ or SWIFT calculated $\mathrm{x}$; and the relative differences between the two distributions.

\subsection{Conclusions}

The Griffin code was used for neutronics calculations in the microreactor multiphysics simulations. Different schemes of solving the neutron transport equations, the SAAF-CFEM-SN, the CFEMDiffusion, the DFEM-SN, and the CFEM-Diffusion and SPH methods, have been applied to the 3D unit cell model.

The low fidelity diffusion method is the fastest to calculate the reactor physics. Due to the highly heterogeneous geometrical model used in the multiphysics simulation, the diffusion method provides significantly different axial power shapes compared to the reference solution, as expected, and overestimated reactivity temperature feedback effects by more than $100 \%$ compared to the reference solution. The diffusion and SPH method has been applied to improve the accuracy of the reactor physics results obtained from the diffusion calculation. With this method the speed of the convergence rate of the diffusion method can preserved in multiphysics simulations, which are described in Section 7, and the accuracy of the solution is preserved: the axial power distributions 
and the reactivity temperature feedback effects have all been calculated accurately. In the future, more work will be required to apply the method for analysis in assembly and full-core models.

The $\mathrm{S}_{\mathrm{N}}$ methods calculated the axial power distributions accurately and can provide very good estimates of reactivity temperature feedbacks during the transient. However, the $S_{N}$ methods currently implemented in Griffin require longer computational times without acceleration schemes. Methods in Griffin to improve the $S_{N}$ method's efficiencies for multiphysics simulations are still under active development. In this study, the $S_{N}$ methods were only used for verification studies on the 3-D unit cell models.

A full core model which has one-sixth of the microreactor core included was developed. The diffusion method was used to demonstrate the workflow of coupling Griffin with other physics codes for transient simulations as applied in Sections 7.3 and 7.4. Future work will include improving the accuracy of the reactor physics calculation in the full core calculation by applying $\mathrm{S}_{\mathrm{N}}$ methods (with acceleration schemes) for the transient simulations.

Multiphysics simulations performed under the MOOSE framework used the piecewise linear functions to take into account the variations of material properties during the transients. For this microreactor, the reactivity feedback effects due to the material temperature variations or the $\mathrm{H}$ content variations in the core were modeled by using the bidimensional cross section tables. Numerical simulations have been performed and verified that the reactor physics can model these effects accurately using multigroup cross sections interpolated from the bidimensional look-up tables. In addition, a simple Griffin model was developed to demonstrate that the Griffin code coupled with SWIFT can be used to model the hydrogen dissociation effects in the reactor physics simulations. 


\section{Bison Fuel and Heat-Conduction Modeling}

Two types of simulations in different length scales (engineering scale in meters vs. microscale in micrometers) using BISON code/models have been performed in this study. First type of simulation contains two cases: microreactor full-core heat-conduction and thermo-mechanical analyses. Figure 5-1 (a) shows 1/6 symmetric microreactor core mesh generated by CUBIT [20]. All components including heat pipes, moderators, fuels, control drums, reflectors, graphite monolith and helium gaps, were modeled in the heat conduction simulation utilizing the mesh shown in Figure 5-1 (a). Figure 5-1 (b) shows the simplified mesh for thermo-mechanical simulation, where helium gaps and control drums were removed in the preliminary study. Noted that for multi-physics simulation (BISON-Griffin-Sockeye), the heat pipes meshes shown in both Figure 5-1 (a) and Figure 5-1 (b) are removed for BISON sub-app simulation as Sockeye is taking over the heat pipe computations. Figure 5-1 (c) shows 2D axisymmetric (2D-RZ) mesh for a standalone TRISO fuel particle developed with "TRISO2DMeshGenerator" model in BISON.

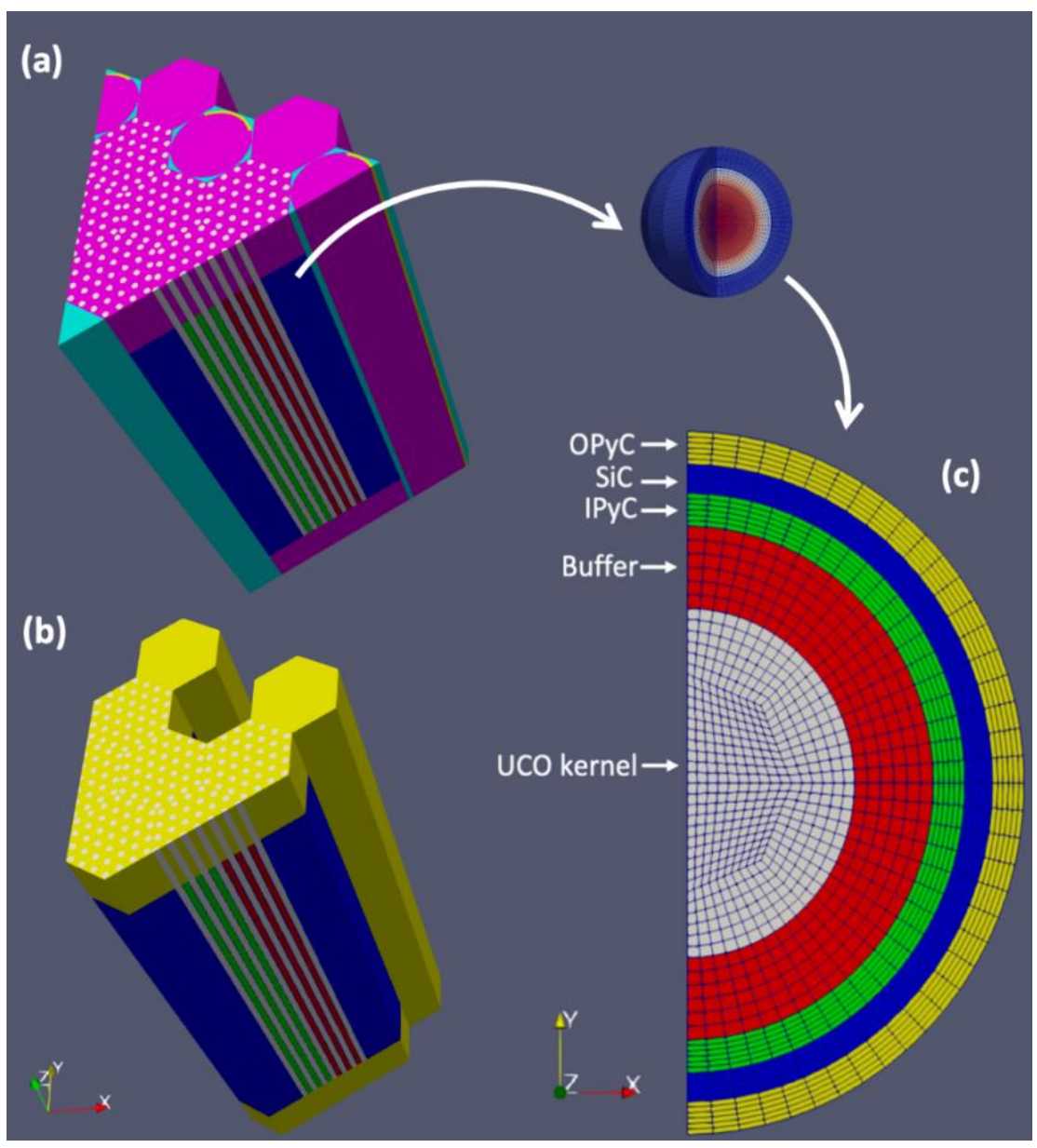

Figure 5-1. Various meshes used in different simulation cases: (a) heat conduction full-core simulation; (b) thermo-mechanical full-core simulation; and (c) 2D-RZ standalone TRISO fuel particle simulation. 


\subsection{Full-core heat-conduction and thermo-mechanical simulation with BISON}

MOOSE contains a set of physics modules that can be built in various applications such as Griffin and BISON. In this simulation, two key physics modules, heat conduction and tensor mechanics, were employed for heat conduction and thermo-mechanical modeling. BISON contains materials' models (thermo and mechanical properties) for stainless steel 316, graphite, and TRISO fuel, all of which are required for the full core thermo-mechanical simulation.

To demonstrate the heat conduction and thermo-mechanical modeling capability of the BISON code, full-core (1/6 symmetric) simulations with no input power was performed. The core was simulated to establish a "steady-state" which is also the starting status of the multiphysics simulation. The temperature of the core at the initial state was $300 \mathrm{~K}$ uniformly. Without input power, the temperature distribution relies on the boundary conditions: the heat pipe surfaces with external temperature of $800 \mathrm{~K}$ are the only heat sources, and external surfaces (including top, bottom and outer surfaces of reflector, and top surface of helium gap) with external temperature of $300 \mathrm{~K}$ become the heat sink.

Figure 5-2 shows the temperature distribution of 1/6 symmetric core at different time spans in the simulation. The core region is heated up by the heat pipes gradually and reached the steady state at $\sim 60 \mathrm{sec}$. The helium gap in the core center, the control drums, and the side reflector regions were maintained at low temperatures as their external surfaces are the heat sinks. Figure 5-3 shows the evolution of the average temperatures of fuel and heat pipe surfaces, both of which gradually developed into $\sim 800 \mathrm{~K}$, indicating the steady state is being established.

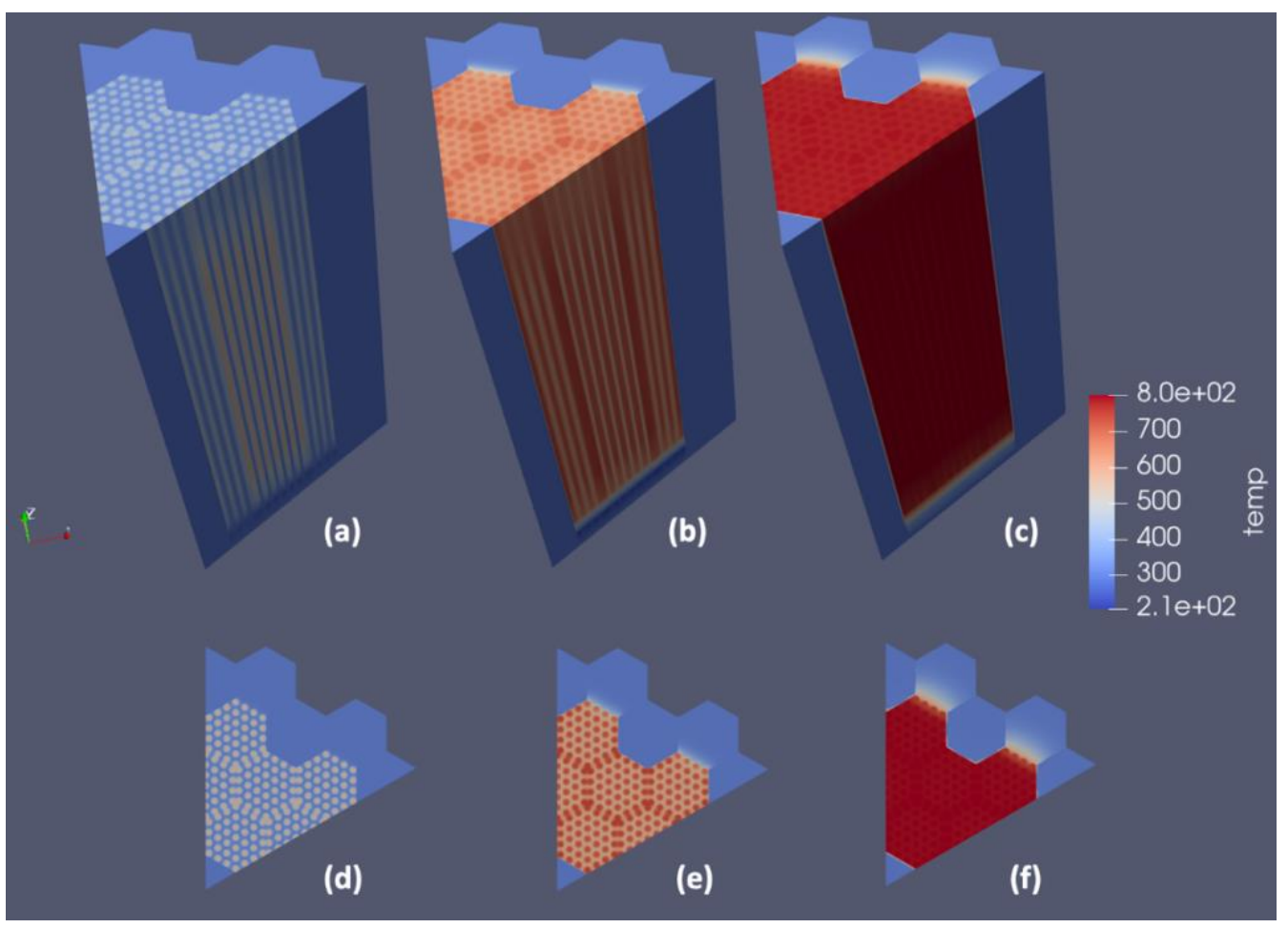

Figure 5-2. Temperature evolution of the microreactor core to establish a steady state: 3D temperature distribution (unit: K) at (a) time $=1 \mathrm{sec}$; (b) time $=10 \mathrm{sec}$; and (c) time $=60 \mathrm{sec} ; 2 \mathrm{D}$ temperature distribution (unit: K) at the core center at (d) time $=1 \mathrm{sec}$; (e) time $=10 \mathrm{sec}$; and (f) time $=60 \mathrm{sec}$. 

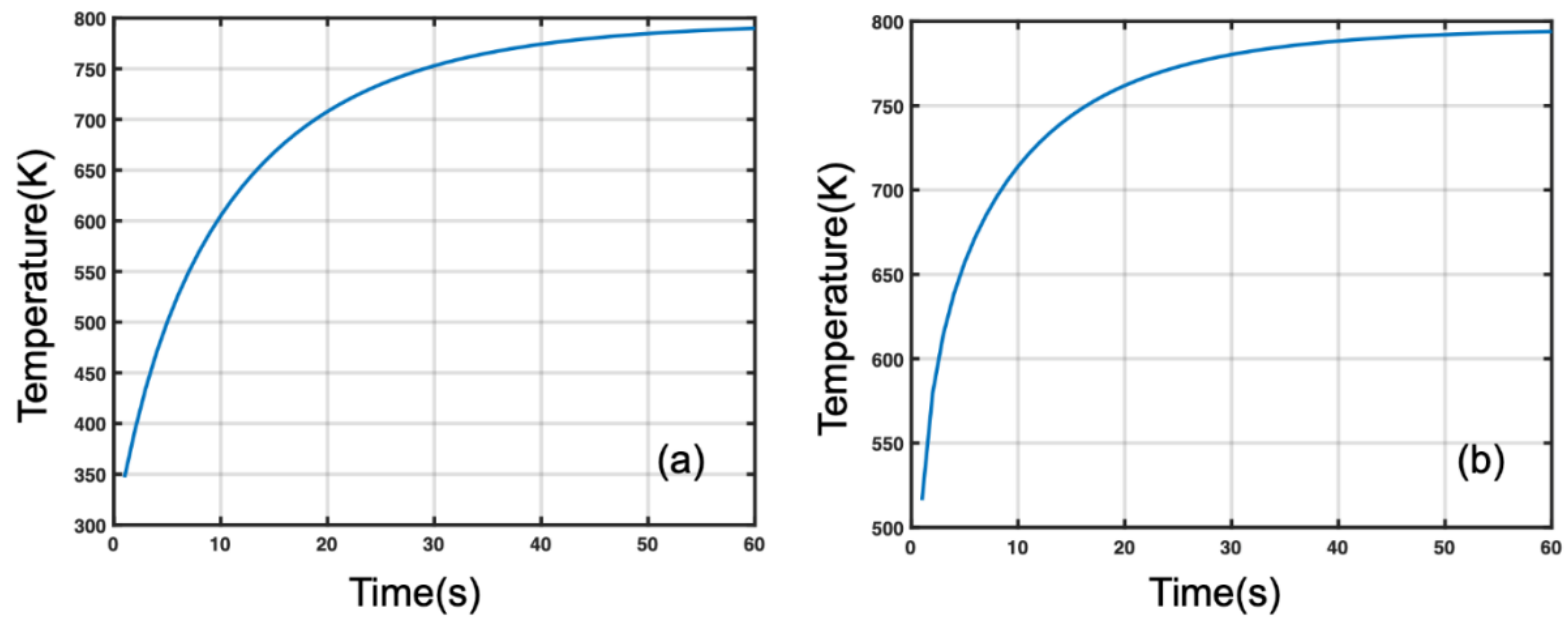

Figure 5-3. Temperature evolution of the microreactor core to establish a steady state: (a) average fuel temperature, and (b) average heat pipe surface temperature.

To demonstrate the BISON's thermal-mechanical modeling capability with coupling heat conduction and elastic mechanics, full-core (1/6 symmetric) simulations with no input power was performed. This simulation is similar to the aforementioned heat conduction problem in terms of thermal boundary conditions, initial temperatures, and other computation parameters (e.g. time step), whereas the mesh employed in the thermal-mechanical simulation is simplified (Figure 5-1 (b)). In addition, the elastic mechanical models were added into the simulation by employing MOOSE's tensor mechanics module. Only the bottom of the core was fixed in all directions (displacement of $x, y, z=0$ ), and other parts of core can be freely expanded. Figure 5-4 (a), (b) and (c) show the displacement (magnitude in unit of meter) distribution at various time spans, and their corresponding temperature distribution were shown in Figure 5-4 (d), (e) and (f). The temperature distribution in thermo-mechanical simulations at different time spans (Figure 5-4 (d), (e) and (f)) are similar to those developed in heat conduction simulations (Figure 5-2 (a), (b) and (c)). By computing the mechanical response of each component, the core was expanded (thermal expansion) due to the temperature increase (Figure 5-4 (a), (b) and (c)). The magnitude of displacement from the initial state $(\mathrm{t}=1 \mathrm{~s})$ to the end state $(\mathrm{t}=60 \mathrm{~s})$ can also be observed in the graphic demonstration in Figure 5-5. 


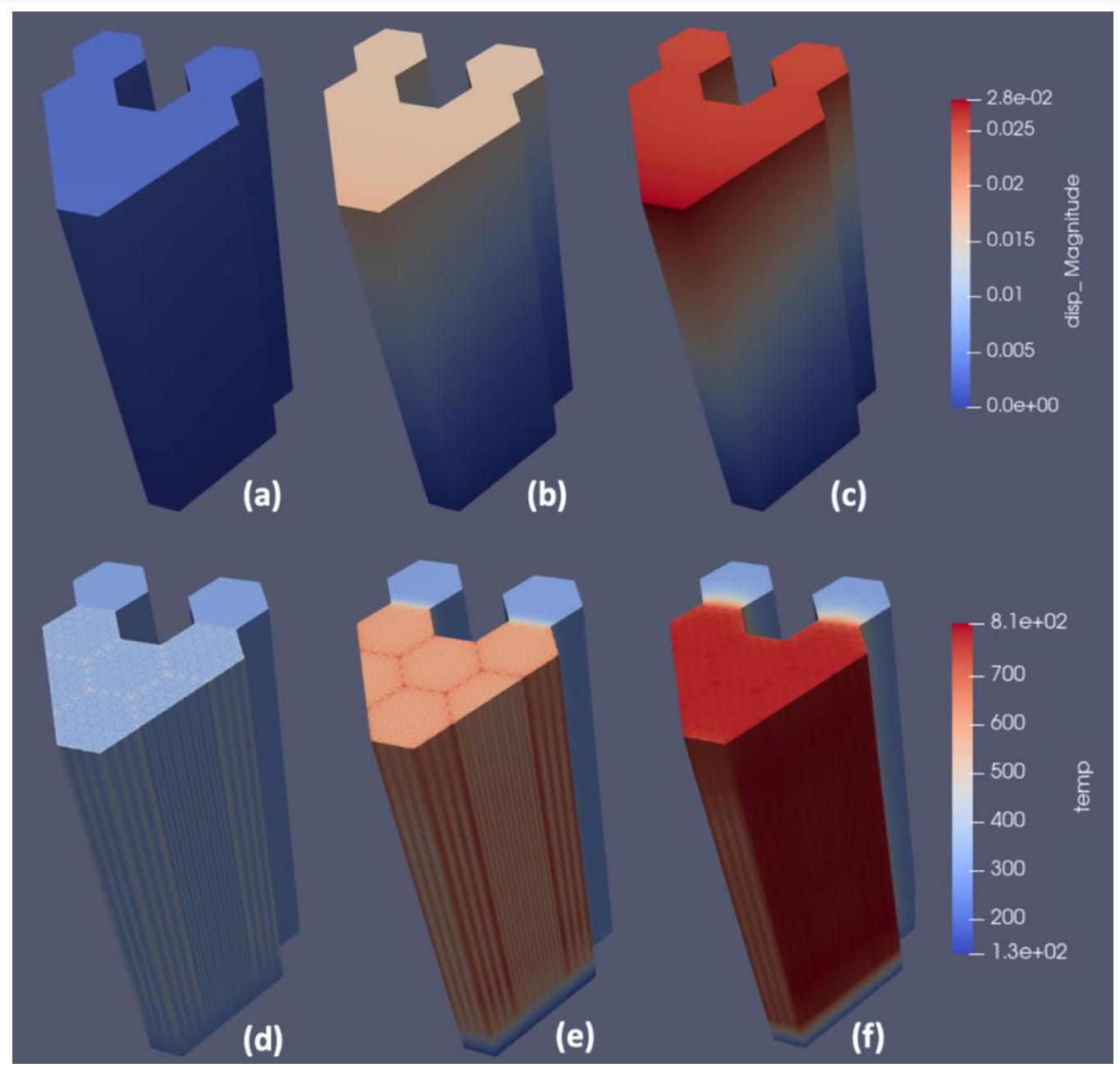

Figure 5-4. Displacement and temperature evolution of the microreactor core during establishing the steady state: $3 \mathrm{D}$ displacement distribution (unit: $\mathrm{m}$ ) at (a) time $=1 \mathrm{sec}$; (b) time $=10 \mathrm{sec}$; and (c) time $=60 \mathrm{sec}$; and 3D temperature distribution (unit: $\mathrm{K}$ ) at (d) time $=1 \mathrm{sec}$; (e) time $=10 \mathrm{sec}$; and (f) time $=60 \mathrm{sec}$. 


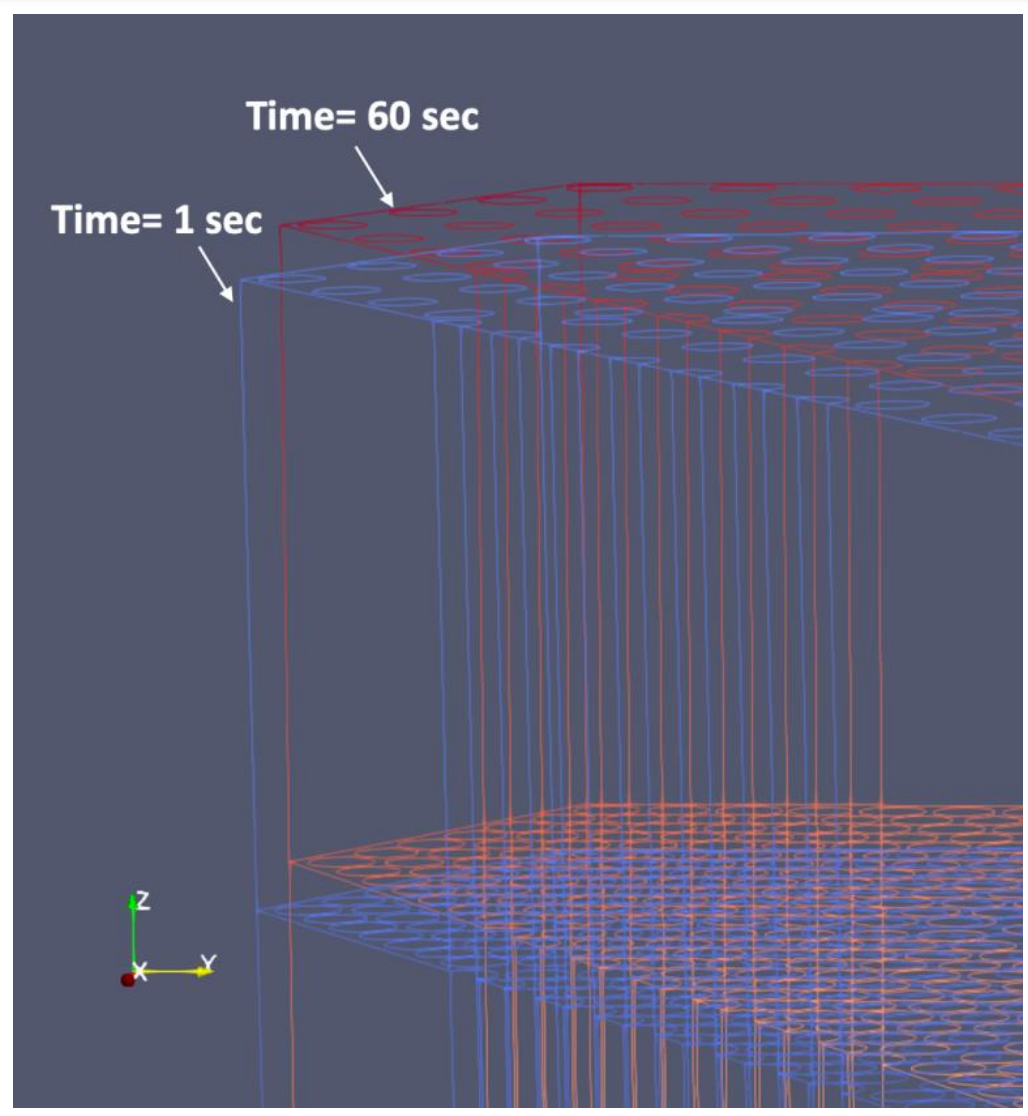

Figure 5-5. Graphic demonstrations of displacement near the top of reflector region.

\subsection{TRISO fuel particle modeling}

TRISO fuel simulation is one of most mature capabilities in BISON. Originally developed with $\mathrm{UO}_{2}$ kernel [26], models of "UCO" kernel have been recently implemented into the BISON code. TRISO models have been benchmarked by comparing the simulation results to PARFUME's [27].

To demonstrate the application of BISON on modeling TRISO fuel particles for supporting microreactor development, a standalone TRISO particle was modeled by implementing the materials properties (density of materials) and the microreactor operating parameters (fission rate, fast neutron flux and temperature). Although BISON is capable of modeling TRISO fuel performance in 1, 2, or 3 dimensions (1,2, or 3D), we simulate a 2D axisymmetric (2D-RZ) TRISO fuel geometry in our preliminary assessment as $2 \mathrm{D}-\mathrm{RZ}$ is sufficient to demonstrate the fuel performance for a perfect particle (defective particles need 2D or 3D modeling to evaluate the impact of defects). Thermal and mechanical properties of UCO, Buffer layer, PyC layers, and SiC layers were implemented either through BISON models or using the recommended values in ref. [28]. The fuel exterior temperature was set to be $900 \mathrm{~K}$. The fission rate of the TRISO is $\sim 1.29 \times 10^{18}$ calculated based on the average flux level in the microreactor design considered in Section 2 . The average fast neutron flux is $7.664 \times 10^{15} \mathrm{n} / \mathrm{m}^{2}$-s. A 10 -year fuel performance simulation was performed to develop an average final burnup of $\sim 1.67 \%$ FIMA $(\sim 17 \mathrm{MWd} / \mathrm{kgHN})$. 


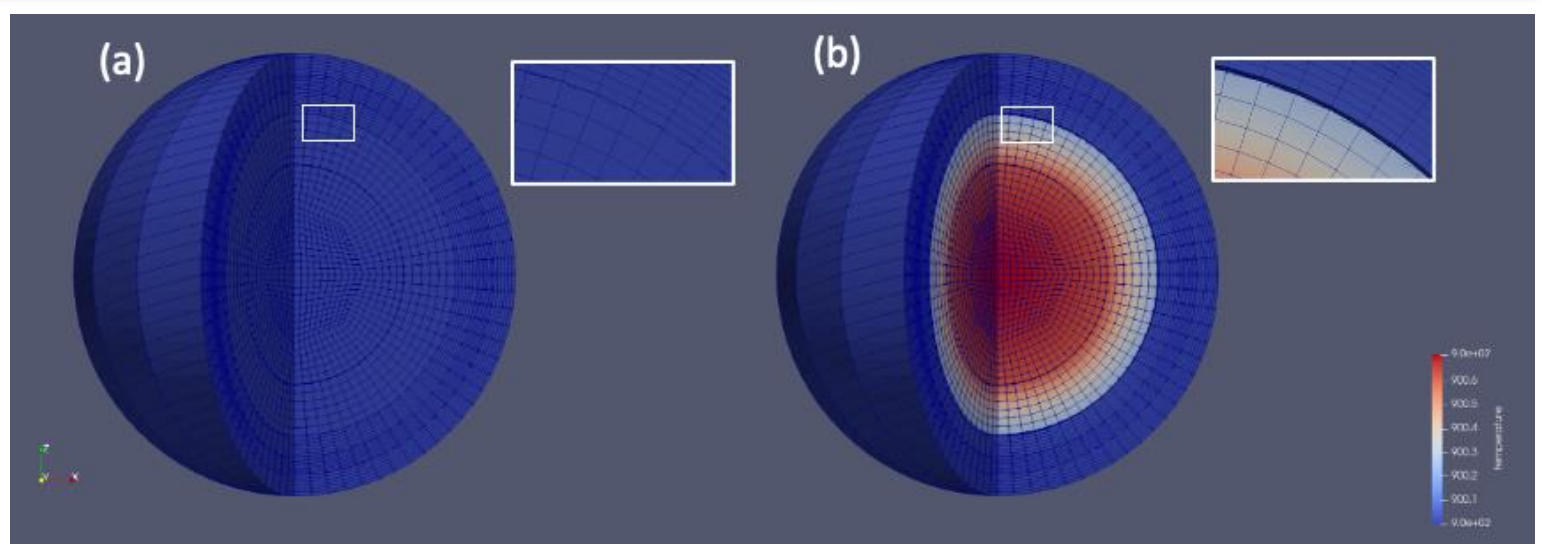

Figure 5-6. Temperature distribution of a TRISO fuel particle in the microreactor at (a) time $=0$, and (b) time $=10$ years. The " $3 \mathrm{D}$ " images were produced based on the $2 \mathrm{D}-\mathrm{RZ}$ simulation results for demonstration.

Figure 5-6 (b) \& (c) show the fuel particle temperature distribution at the start (time $=0$ ) and the last time step of simulation (time $=315.4$ Ms or 10 years), respectively. As seen, after 10 years of irradiation, despite a low fast neutron flux, a small gap was generated between the buffer and IPyC layer. Meanwhile the temperature gradient within the TRISO fuel particle is negligible due to the low power density/fission rate. The temperature at the fuel kernel center is $900.7 \mathrm{~K}$, only $0.7 \mathrm{~K}$ higher than the external boundary temperature $(900 \mathrm{~K})$.

Fission gas release and particle pressure development of a TRISO fuel particle during the 10 years of irradiation in the microreactor were found to be moderate but with consistent order of magnitude when compared with literature [26]. Fission gas release shown in Figure 5-7 is $\sim 6.5 \times 10^{-10}$ moles after 10 years of irradiation as a result of the low burnup, fast neutron flux and temperature in microreactor. The tangential (hoop) stresses built in the IPyC, OPyC and $\mathrm{SiC}$ during irradiation were shown in Figure 5-7 (b). As expected, the IPyC and OPyC layers were in tension due to the shrinkage, which caused the $\mathrm{SiC}$ in compression. Further irradiation may result in relaxation of the stresses due to irradiation creep [26]. According to the preliminary simulation, the integrity of fuel particle is well preserved after 10 years of irradiation in the microreactor operating conditions. The example in this study demonstrated the capability of BISON for performing the TRISO fuel particle analysis for microreactor applications. 


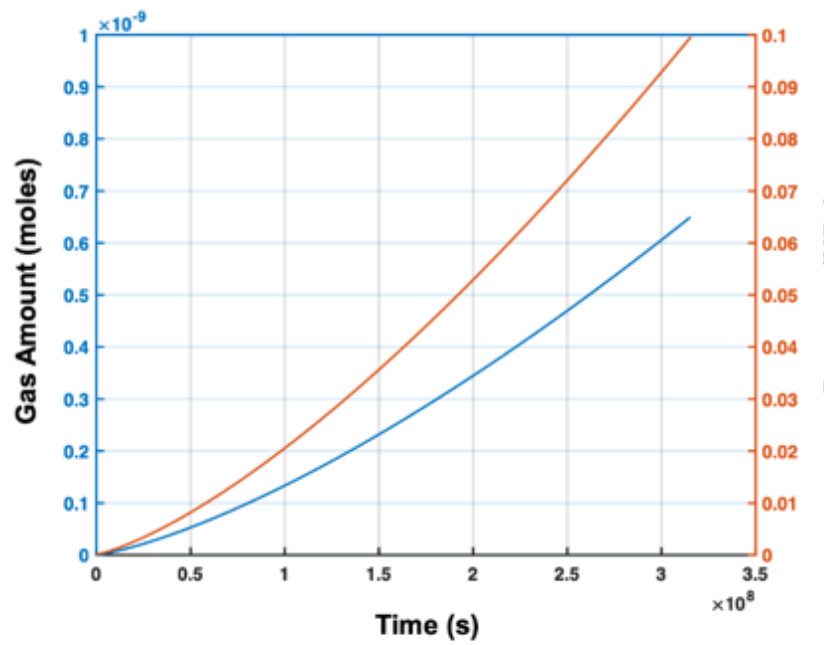

(a)

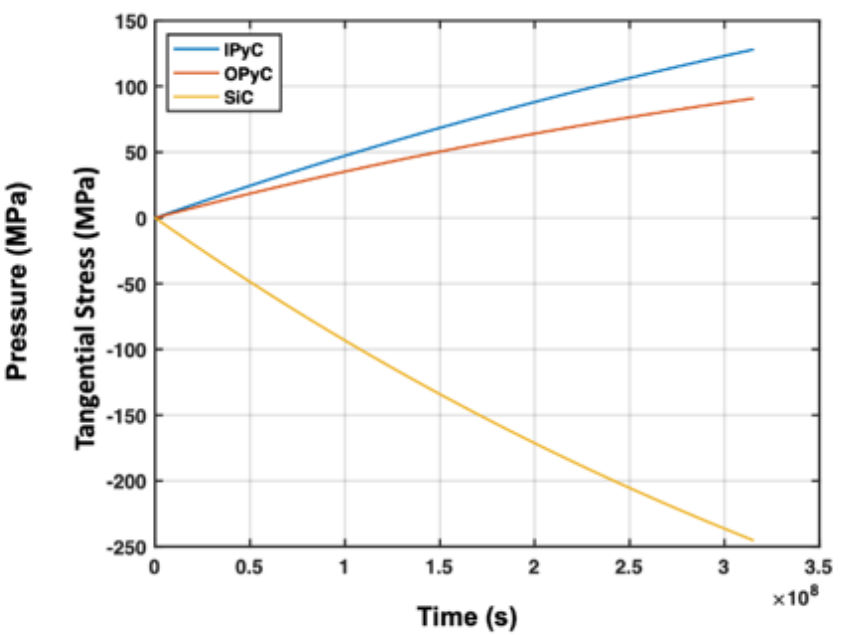

(b)

Figure 5-7. Computed histories of (a) fission gas release and particle pressure and (b) maximum tangential stress of IPyC, OPyC and SiC. End of irradiation (10 years) corresponds to a burnup of $\sim 17 \mathrm{MWd} / \mathrm{kgHN}$. 


\section{Heat-pipe modeling}

The Sockeye code is used for heat conduction modeling within the heat pipe, in both single-physics simulations to ensure adequacy of the heat pipe design of the HP-MR, and for multiphysics simulations discussed in Section 7. Two models were used for the heat-conduction analyses in the heat pipe of the micro-reactor model: one employing the two-phase thermal-hydraulic model and one employing the effective thermal conduction model. General design parameters are provided in Table 6-1, with a schematic in Figure 6-1 (a). The length of the evaporator section corresponds to the combined height of the fuel and upper reflector of the micro-reactor. The energy conversion system has not yet been considered in our model. A convective heat transfer boundary condition is applied to the condenser section, with an external ambient temperature of $800 \mathrm{~K}$ and a heat transfer coefficient that is sufficiently high $\left(10^{6} \mathrm{~W} / \mathrm{m}^{2}-\mathrm{K}\right)$ to approximate a fixed temperature boundary condition. In the single-physics model, a uniform heat flux is applied to the evaporator section, such that the total heat rate is $1,800 \mathrm{~W}$.

Table 6-1. Parameters in sockeye heat pipe performance model.

\begin{tabular}{|l|c|r|}
\hline \multicolumn{1}{|c|}{ Parameter } & Units & \multicolumn{2}{c|}{ Value } \\
\hline Envelope material & - & \multicolumn{2}{c|}{ SS 316} \\
\hline Coolant & $\mathrm{cm}$ & \multicolumn{1}{c|}{ Potassium } \\
\hline Outer radius & $\mathrm{cm}$ & 1.05 \\
\hline Length of evaporator section & $\mathrm{cm}$ & 30.00 \\
\hline Length of adiabatic section & $\mathrm{cm}$ & 90.00 \\
\hline Length of condenser section & $\mathrm{cm}$ & 0.08 \\
\hline Thickness of envelope & $\mathrm{cm}$ & 0.07 \\
\hline Thickness of annulus & $\mathrm{cm}$ & 0.10 \\
\hline Thickness of wick & $\mu \mathrm{m}$ & 15 \\
\hline Pore Radius & - & $2 \times 10^{-9}$ \\
\hline Permeability & - & 0.70 \\
\hline Porosity & & \\
\hline
\end{tabular}

Heat removal capacity of a heat pipe may be limited by any of several phenomena, which depend on the operating temperature of the coolant in the heat pipe. Sockeye provides estimates of the capillary, sonic, viscous, entrainment, and boiling limits by evaluating analytic functions for each limit. These functions depend on the heat pipe geometry, wick properties, and coolant properties. A Sockeye utility was employed to evaluate each operating limit as a function of temperature, and the plot of this data is provided in Figure 6-1 (b). The plot includes a point labeled as the "Operating Condition" at the lower limit on coolant temperature set by the boundary condition of $800 \mathrm{~K}$ and a target heat removal capacity of $1,800 \mathrm{~W}$, corresponding to the mean heat pipe removal capacity in the micro-reactor core. From the plot, the heat pipe would be expected to be operable up to at least $3,000 \mathrm{~W}$ when it reaches the sonic limit. 


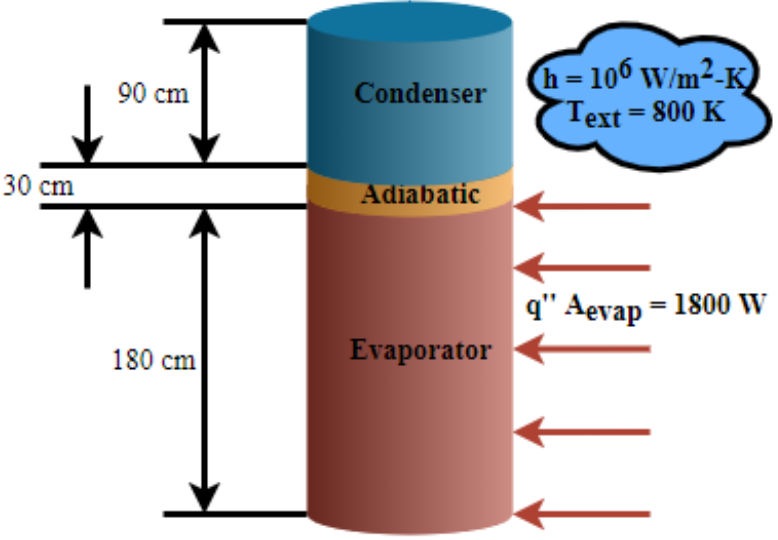

(a)

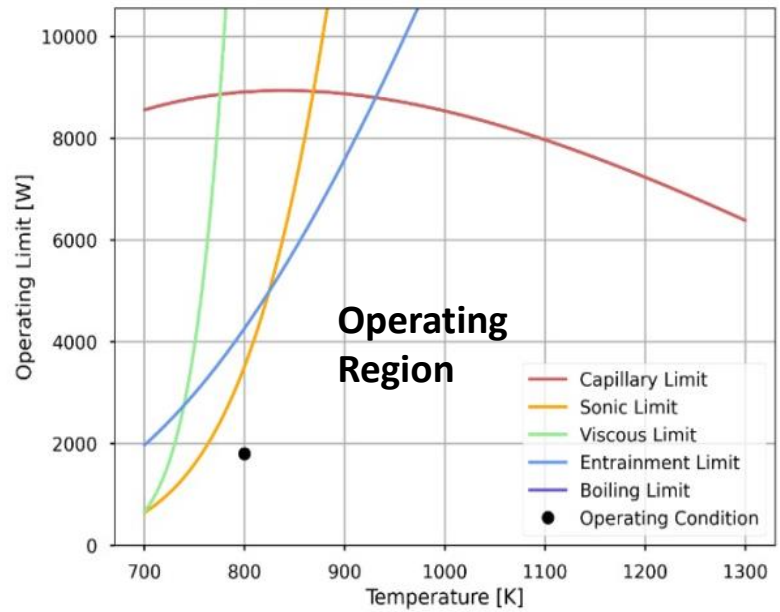

(b)

Figure 6-1. (a) Schematic of heat pipe model with boundary conditions (not to scale), (b) operating limits of heat pipe as computed by Sockeye utility.

Predictions of the heat pipe void fraction at steady-state, normal operating conditions using the twophase model are provided in Figure 6-2 (a). In the evaporator section (left on the plot), the void fraction corresponds to a liquid annulus and wick and a dry vapor core. In the condenser section (right on the plot), some liquid begins to enter the central core of the heat pipe. The axial profile of temperature is provided in Figure 6-2 (b), comparing the results of the two-phase to those of the effective conduction model. The two-phase model provides temperature for both the liquid and vapor phases of the potassium coolant inside the heat pipe. In the effective conduction model, the thermal conductivity of the vapor core region was calibrated to $1.3 \times 10^{6} \mathrm{~W} / \mathrm{m}-\mathrm{K}$ to achieve good agreement with the two-phase model in the evaporator region. In the two-phase model, the decrease in void fraction in the condenser section corresponds to reduced coolant temperatures, with the minimum void fraction corresponding to a point of inflection in the temperature profile. The effective conduction model excludes this physics, and instead predicts a smooth temperature profile in the condenser section.

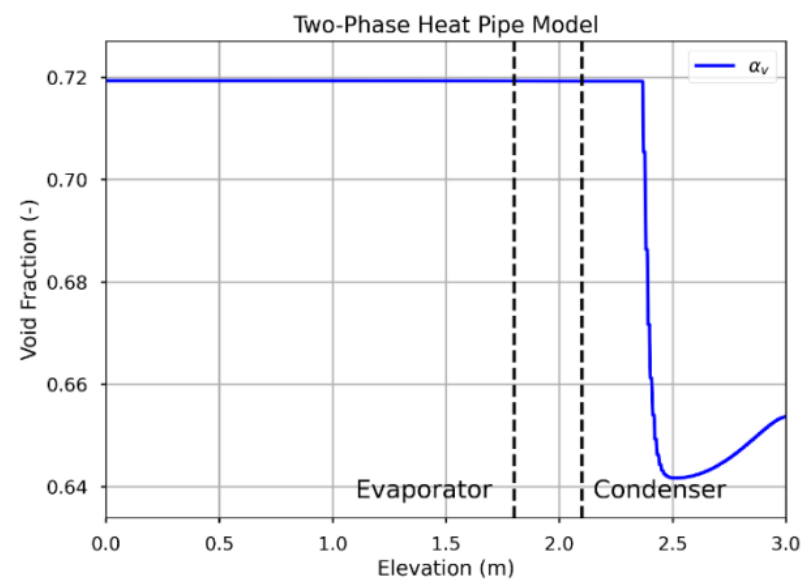

(a)

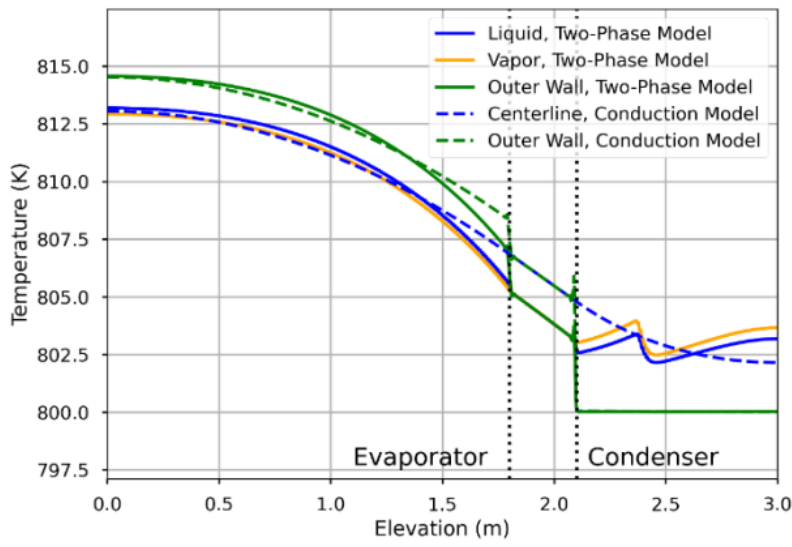

(b)

Figure 6-2. (a) Axial void fraction profile and (b) temperature profiles predicted by Sockeye at stead-state, normal operating conditions by the two-phase model. 


\section{Multiphysics transient analyses}

Different multiphysics transient scenarios were simulated using various approaches for both unit cell and 1/6-core meshes of the HP-MR. Those mostly rely on the Griffin, BISON, and Sockeye codes discussed in previous sections. They are coupled through the MultiApp approach described in Figure 7-1. For the unit cell analysis in Section 7.1, the focus was on applying the diffusion and SPH method in the multiphysics simulations. For the 1/6-core case simulations in Section 7.2 to 7.4 , both load-following and single heat pipe failure were investigated using the diffusion method to demonstrate the capabilities of the MOOSE multiphysics frameworks, while relying on simplified Diffusion-only neutronic simulations. Finally, decay heat removal simulation through RCCS system is discussed in Section 7.5 using SAM/MOOSE coupling.

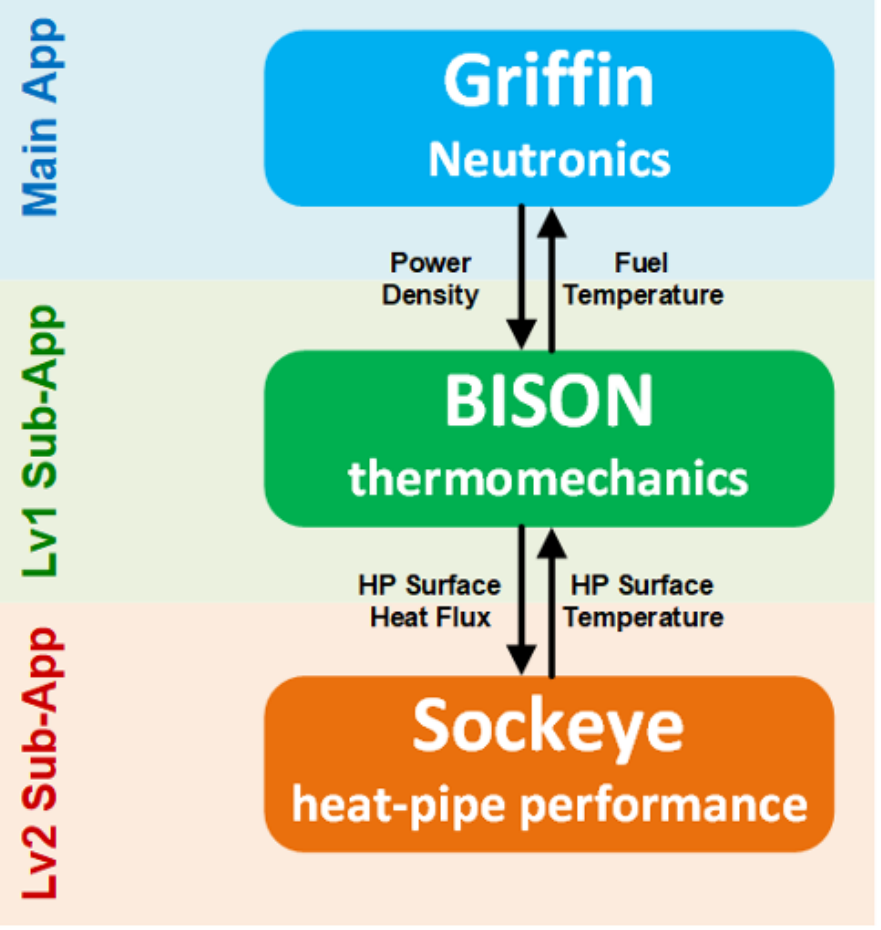

Figure 7-1. Typical MultiApp strategy for micro-reactor multiphysics simulations.

\subsection{Load following (unit cell)}

Reactor physics calculation using the diffusion and SPH method in Griffin was coupled with BISON and Sockeye to enable multiphysics simulations of both steady state and load following transient scenarios for a unit cell of a heat-pipe microreactor. The results are compared with simulations of a similar Griffin-BISON-Sockeye multiphysics simulation using the diffusion only method.

The Griffin-BISON-Sockeye multiphysics simulation framework shown in Figure 7-1 is based on the MOOSE MultiApps system. It adopts Griffin as the main application to solve neutronics equations. BISON is used as the first-level sub-application to solve heat conduction equations in unit cell fuel, monolith, moderator, and reflector, while Sockeye is used as a second-level subapplication under BISON to govern heat pipe related thermal problems. Different levels of MultiApps system are coupled through Picard iteration. The Griffin main application solves 
neutronics equations and transfers the corresponding power density profile to the BISON subapplication. On the other hand, the BISON sub-application computes temperature profile and feedbacks the temperature information back to the main application.

Depending on the different cross section look-up tables used in the Griffin reactor physics calculations, four different multiphysics simulations were investigated for this unit cell, which are described in detail as follows:

(1) The 1d-grid look-up table described in section 4 is used to generate the temperature dependent multigroup cross sections. The grid variable is the fuel temperature. For the fuel mesh block, the local temperature calculated by BISON is used to tabulate the cross-section. For the non-fuel mesh blocks, the temperature at the nearest fuel surface positions is used to tabulate the cross-section. This approach is termed "1D Surface" in this report.

(2) Similar to case (1) except that a layer-averaged fuel temperature is calculated at each axial position and is used as the fuel temperature for the non-fuel mesh blocks to tabulate the cross-sections. This approach is termed "1D Average" in this report.

(3) The 2d-grid look-up table described in section 4 is used to generate the temperature dependent multigroup cross sections. The grid variables are the fuel and moderator temperatures. The fuel temperature within each mesh block is determined similarly to the "1D Surface" case. For the moderator temperature, in the moderator mesh block, the local temperature calculated by BISON is used to tabulate the cross-section. For the nonmoderator mesh blocks, the temperature at the nearest moderator surface positions is used to tabulate the cross-section. This approach is termed "2D Surface" in this report.

(4) Similar to case (3) except that the fuel temperature within each mesh block is determined similarly to the "1D Average" case. For the moderator temperature, in the moderator mesh block, the local temperature calculated by BISON is used to tabulate the cross-sections. For the non-moderator mesh blocks, a layer-averaged moderator temperature is calculated at each axial position and is used to tabulate the cross-sections. This approach is termed " $2 \mathrm{D}$ Average" in this report.

Approach (1) is regarded as the base case. The motivation of investigating cases using the $2 \mathrm{~d}$-grid look-up table originates from the concern that the fuel temperature variations might not be able to represent the temperature variations in other regions during a transient. The "surface" approach is the "default" option in MOOSE if not specified to interpolate the cross sections. The reason to replace this "default" option with the layer-averaged temperature in looking-up cross sections is because the layer-averaged approach provides one clear definition for grid value and intuitively it is also a better representation of the actual physics. 


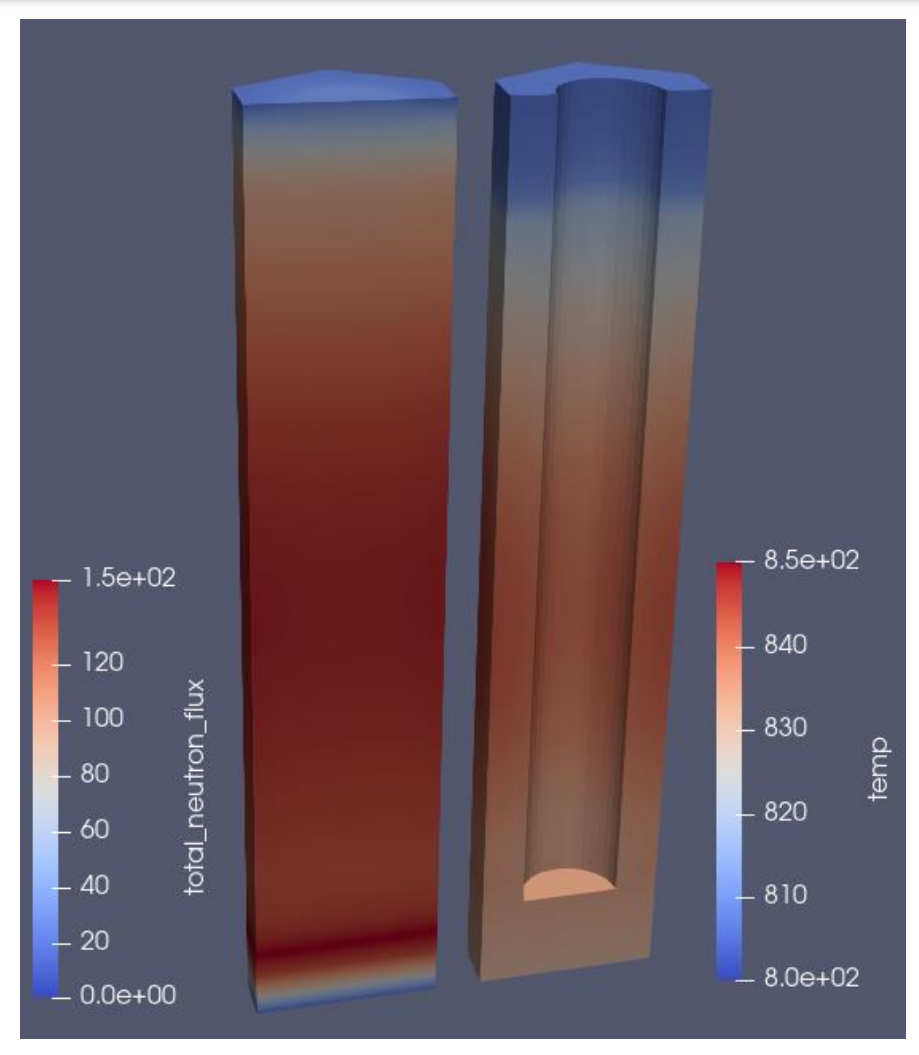

Figure 7-2. A typical steady state reactor physics (total neutron flux) and heat conduction simulation result (material temperature) of multiphysics simulation.

The unit cell simulation was established using a $800 \mathrm{~K}$ secondary loop temperature and a $10^{6}$ $\mathrm{W} /\left(\mathrm{m}^{2} \mathrm{~K}\right)$ convective heat transfer coefficient to provide a nearly Dirichlet boundary condition on the secondary loop surface. At the steady state, as the temperature difference in the radial direction is marginal, the results predicted by the four SPH approach are quite similar (difference in temperature < $0.1 \mathrm{~K}$ ). A typical result of such approaches can be found in Figure 7-2. On the other hand, similar to the results shown in Figure 4-3, the diffusion approach, which was performed with the 1d-grid cross section look-up table, calculated a wrong axial power profile.

To initiate the load following transient, the convective heat transfer coefficient at the secondary loop side is reduced from $10^{6} \mathrm{~W} /\left(\mathrm{m}^{2} \mathrm{~K}\right)$ to $10^{2} \mathrm{~W} /\left(\mathrm{m}^{2} \mathrm{~K}\right)$ (i.e., by $\left.99.99 \%\right)$. This approach is expected to reduce heat removal capability of the heat pipe, thus increasing the unit cell temperature globally and reducing the power through negative feedback reactivity effects. The power and average fuel temperature responses to the reduction in secondary loop cooling capacity that were predicted by all the four aforementioned cases with the diffusion and SPH method are illustrated in Figure 7-3 and Figure 7-4, respectively, in comparison to the diffusion results. 


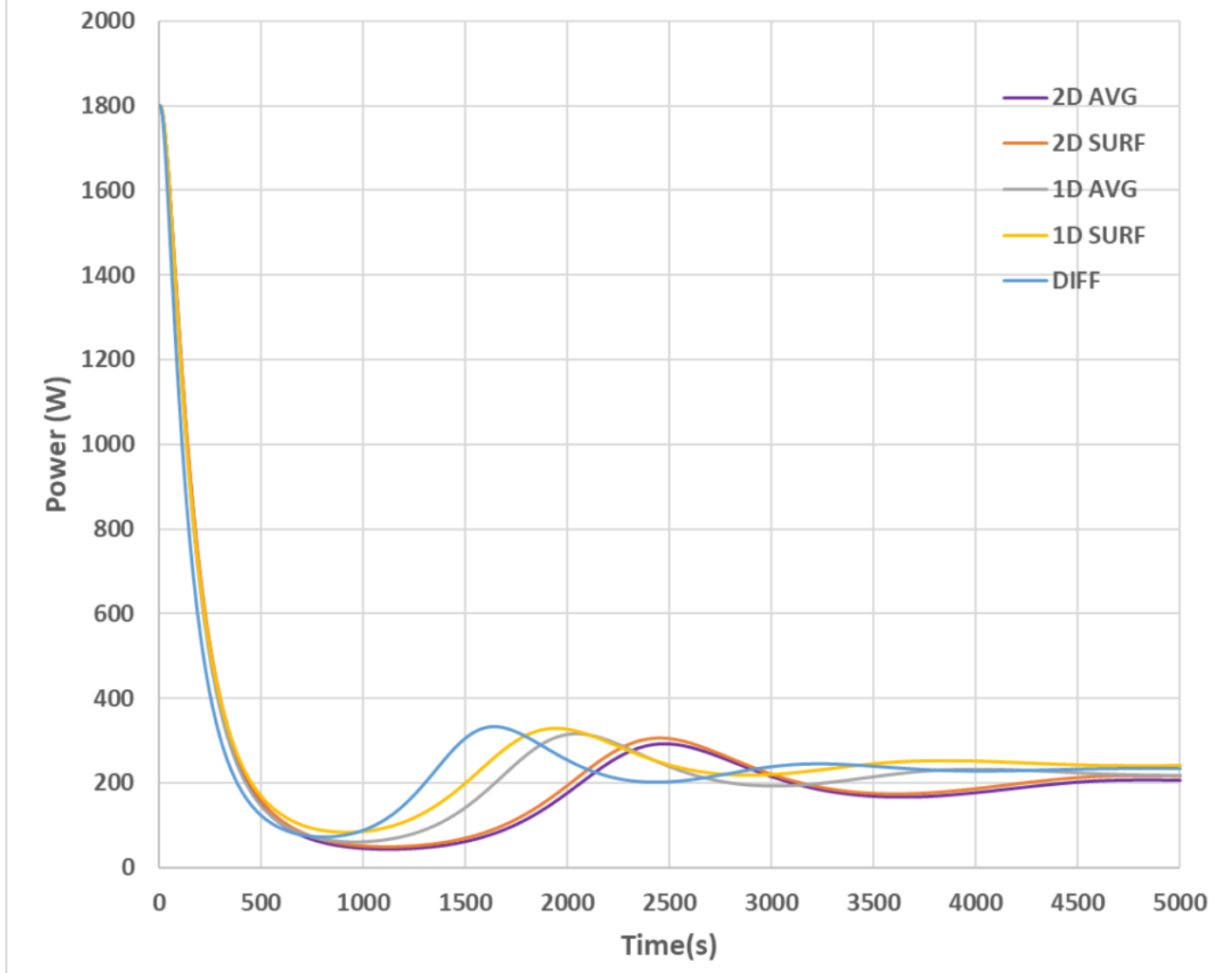

Figure 7-3. Time-evolution of the total unit cell power during load-following power transient predicted by different approaches.

For all the neutronics approaches, the power drops rapidly in the first $\sim 400$ seconds with the corresponding maximum fuel temperature reaches its peak. After that, the system power experiences several oscillations and eventually reaches the new steady state beyond 5,000 seconds. The diffusion-only method predicts a faster power transient compared with results from the other four cases using the diffusion and SPH method, due to the overestimated reactivity temperature feedback effects from the diffusion method as shown in section 4. All the four cases with the SPH method have very similar behavior in the first 400 500 seconds. After that, the results start to differ. The 1D tabulated cross-section approach usually leads to a shorter relaxation time compared to the 2D tabulated cross-section approach, indicating that the distinction between the fuel and moderator temperatures is necessary for more accurate prediction of transient kinetics. On the other hand, the "surface approach" also predicts slightly faster transient compared to the "average approach".

This faster transient is due to the slightly larger k-eff resulted from the "surface approach" during some period of the transient. The temperature gradient from the fuel region to the moderator region is small at the initial steady state $(\sim 3 \mathrm{~K})$ and decreases while the reactor power is reduced. Therefore, in the first 400 500 seconds, the differences among the "average approach" and the "surface approach" is small, and the power transients are the same as shown in Figure 7-3. When the reactor power starts to bounce back, the temperature gradient will increase since the fuel is always heated first. Then, during this period, the "surface approach" always uses a lower "fuel 
temperature" in the nonfuel region and a higher "moderator temperature" in the fuel region compared with the "average approach". As a result, the k-eff of the case using the "surface approach" will be higher than the case using the "average approach" based on the reactivity curves shown in Figure 4-5 during this period of the transient. A higher k-eff leads to a faster power recovery as shown in Figure 7-3. The differences among the two cases diminishes again once the reactor power starts to decrease as being confirmed in Figure 7-3.

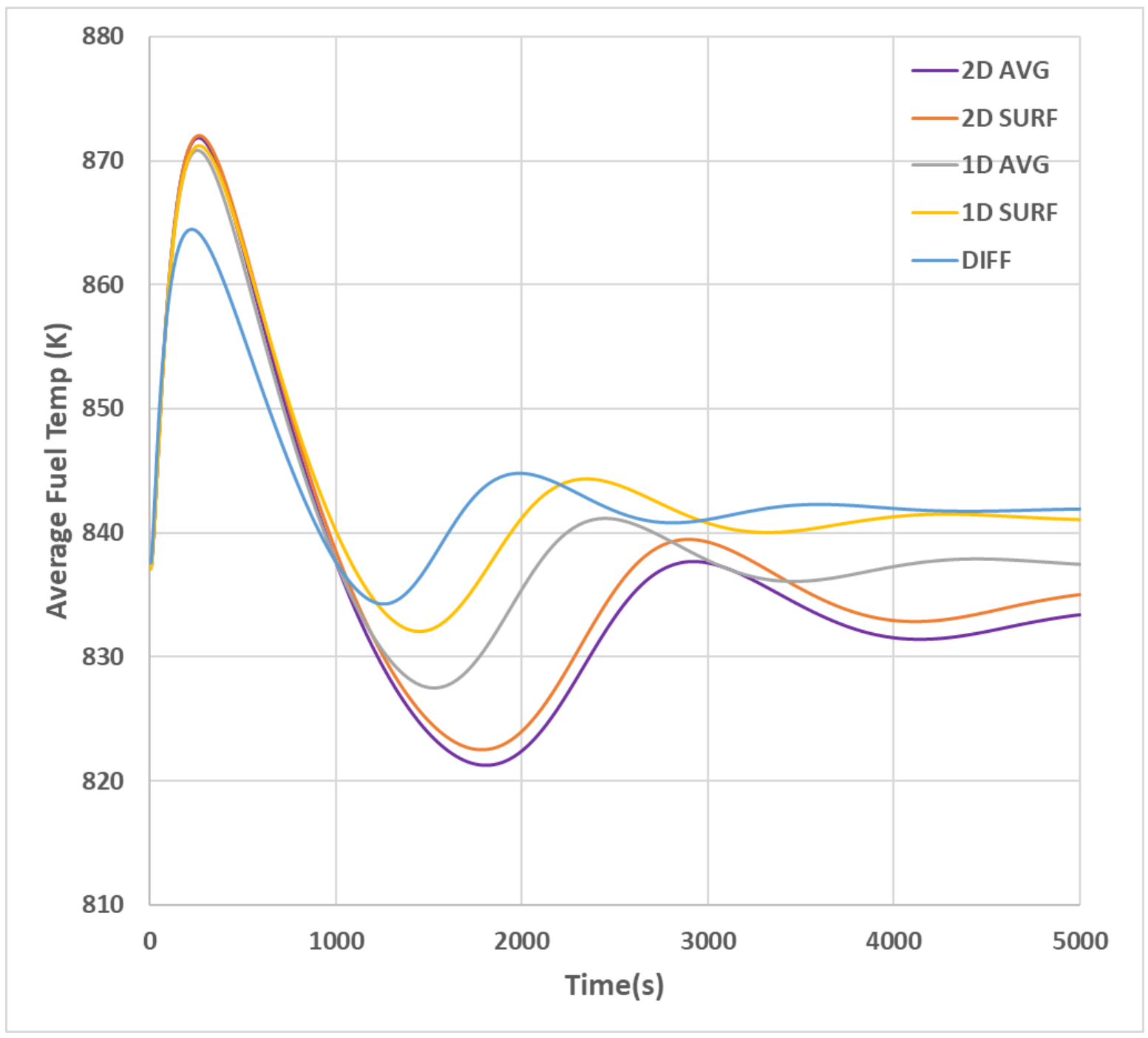

Figure 7-4. Time-evolution of the average fuel temperature during load-following power transient predicted by different approaches.

\subsection{Steady state as initial condition (full-core)}

Based on the success of the unit cell simulation, the Griffin-BISON-Sockeye multiphysics simulation was expanded to full-core simulation. The coupling strategy used in the full-core simulations was derived from the unit-cell simulation strategy. As 192 heat pipes are contained in $1 / 6$ of the simulated microreactor, 192 independent Sockeye second-level sub-applications are used 
to simulate these heat pipes separately, which are governed by a single BISON first-level subapplication.

Steady state simulation was performed first to provide initial conditions for power transient simulations. In the current multiphysics simulations, the BISON sub-application was only used to solve the heat transfer problem instead of thermo-mechanics. At this stage, diffusion neutronics equations were solved in the Griffin main application (without SPH) to provide demonstration of the multiphysics simulation framework. This will be expanded to more advanced neutronics approaches (i.e., transport or diffusion and SPH) in future assessment work.

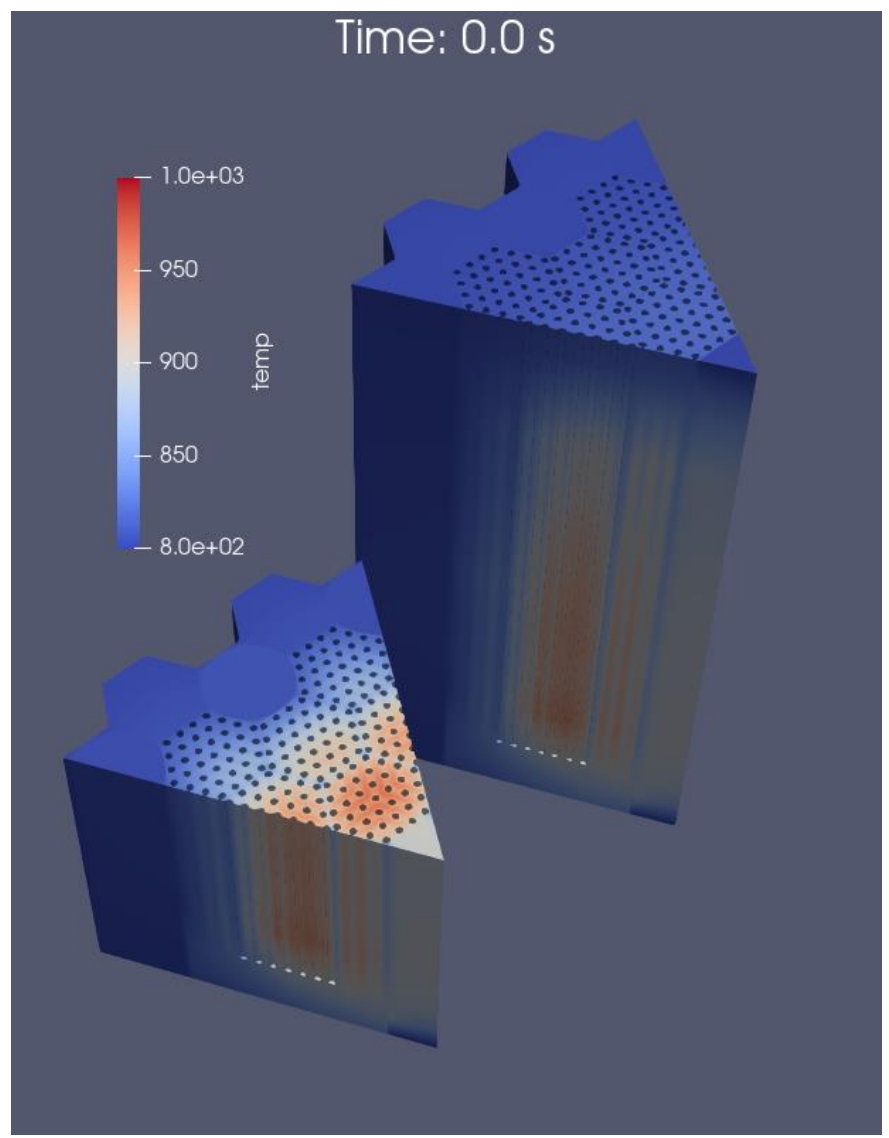

Figure 7-5. Temperature distribution of the 1/6-core at steady state based on the Griffin-BISONSockeye simulation with thermal only BISON approach.

To challenge the design of the HP-MR, the power of the 1/6-core was set as 607,000 W (which is $\sim 82 \%$ higher than reference power of $2 \mathrm{MWt}$ for the full core). This power increase is especially meant to showcase heat pipe cascading failures in Section 7.4 by moving the heat pipe closer to their operating limits. The temperature profile predicted by the multiphysics simulation at steady state is illustrated in Figure 7-5. Both the reactor outer surface convection reference temperature and the heat pipe secondary loop temperature were set at $800 \mathrm{~K}$, which determined the minimum temperature shown in Figure 7-5. The maximum temperature of the steady-state system is observed near the middle of the central assembly of the 1/6-core in the fuel region, which is approximately $980 \mathrm{~K}$. 
As a preliminary trial, the tensor mechanics was also taken into account in a separate simulation. The approach that involves tensor mechanics is termed the "thermo-mechanics" approach in comparison with the conventional "thermo-only" approach. Introducing tensor mechanics into the Multiphysics simulation makes it possible to model thermal expansion of different reactor components while identifying the stress localization positions within the reactor, which facilitates the design optimization to prevent component failure. Also, tensor mechanics can provide a more accurate prediction of local displacement field for neutronics. The calculated displacement field is visualized in the left sub-figure Figure 7-6. The maximum displacement is approximately $1.5 \mathrm{~cm}$. A local von Mises stress field in the monolith matrix is also shown in the right sub-figure of Figure 7-6. The maximum stress in the monolith is found between the neighboring heat pipes, which mainly originates from the local low temperature in monolith due to the high local heat sink strength due to neighboring heat pipes.

It is noticeable that this preliminary trial needs several improvements in the future. The current thermo-mechanics approach takes significantly higher computation resource compared to the thermal-only model, especially in terms of memory usage. Thus, more advanced computer clusters may be used to enable finer meshing. Additionally, effective elastic moduli may need to be used for some components to account for gaps/buffers between dissimilar components that cannot be included in the core mesh. Use of such effective elastic moduli may also help focus the thermomechanics analysis on those key components such as the graphite monolith.

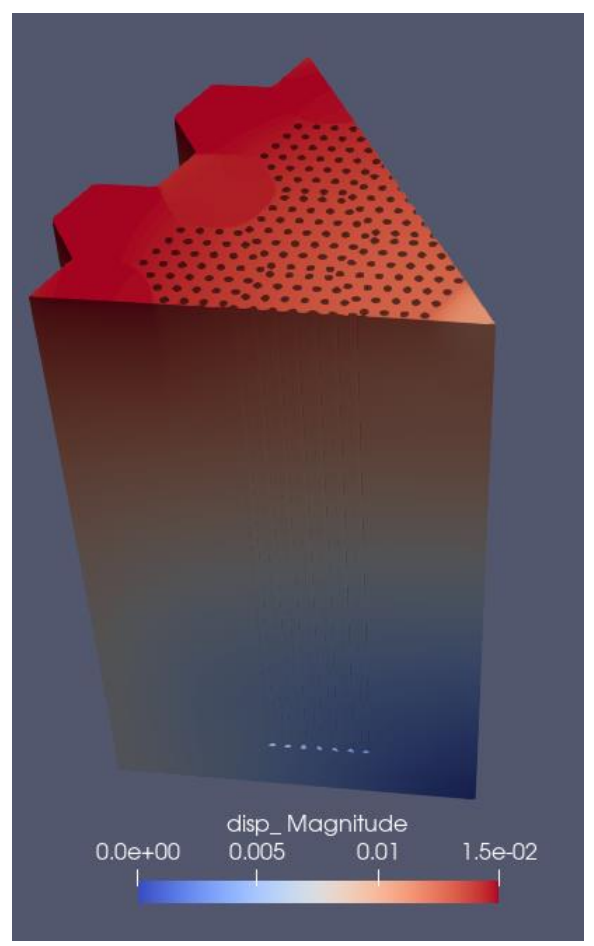

(a)

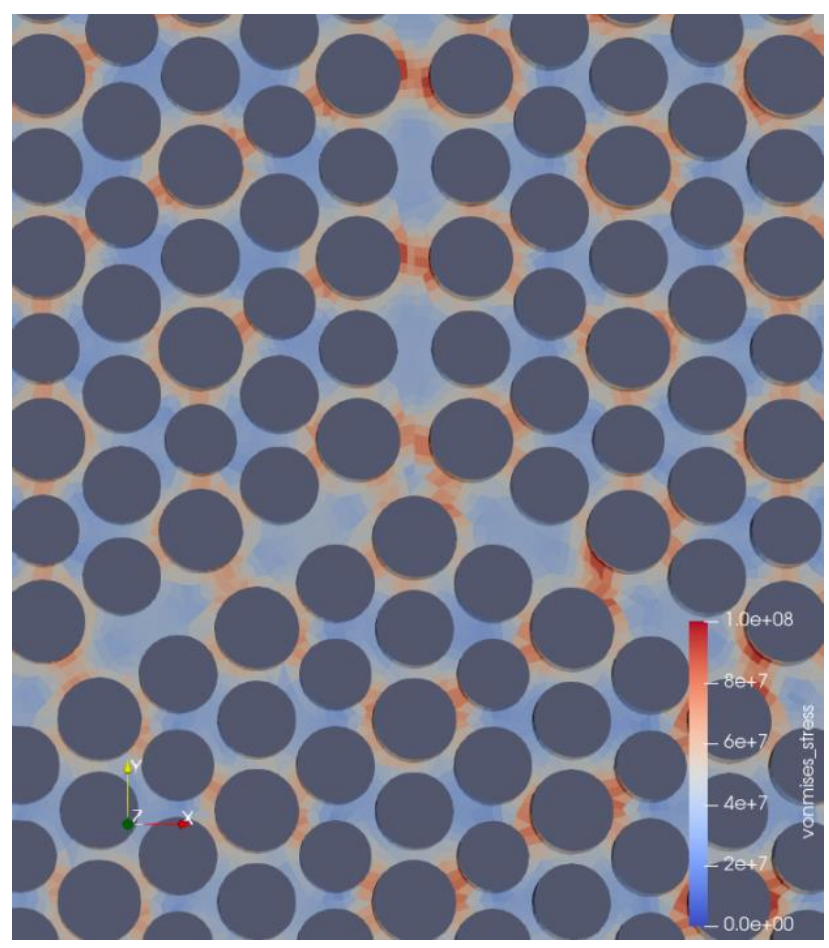

(b)

Figure 7-6. (a) Displacement field and (b) local von Mises stress of the monolith matrix due to thermal expansion of the 1/6-core at steady state based on the Griffin-BISON-Sockeye simulation with thermo-mechanics BISON approach. 
Therefore, additional future efforts will need to eventually enable the multiphysics power transient simulation involving thermo-mechanical models in the BISON sub-application. In this report, all the 1/6-core power transient scenarios simulations were based on the steady-state Griffin-BISONSockeye simulation results involving thermal-only BISON sub-applications (Figure 7-5).

\subsection{Load following power transient (full-core)}

The load following power transient simulation is initiated using an approach similar to the unit-cell load following power transient simulation discussed in Section 7.1. That is, the convective heat transfer coefficient at the secondary coolant loop side of all the 192 heat-pipe in the 1/6 core was reduced from the original $10^{6} \mathrm{~W} /\left(\mathrm{m}^{2} \mathrm{~K}\right)$ to $10^{2} \mathrm{~W} /\left(\mathrm{m}^{2} \mathrm{~K}\right.$ ) (i.e., by $\left.99.99 \%\right)$ at the beginning of the power transient to simulate the reduction in heat removal capacity.

After the reduction in heat transfer coefficient, the time evolutions of the fuel temperature as well as the total 1/6-core power are plotted in Figure 7-7. It is prominent that the reactor power decreases rapidly after the load following transient initiates, while the maximum fuel temperature occurs around 100 seconds after the transient initiation. The maximum fuel temperature is only $\sim 20 \mathrm{~K}$ above the steady state value, implying the large negative temperature reactivity coefficient. The power and temperature then experience a few oscillations and reach the new steady state around 1500 seconds after the transient initiation. Once again, Griffin was applied in this multiphysics modeling exercise using its Diffusion solver (without SPH correction) which we know (see Section 7.1) won't provide accurate results on this heterogeneous core model. The temperature profiles at these representative time points are shown in Figure 7-8.
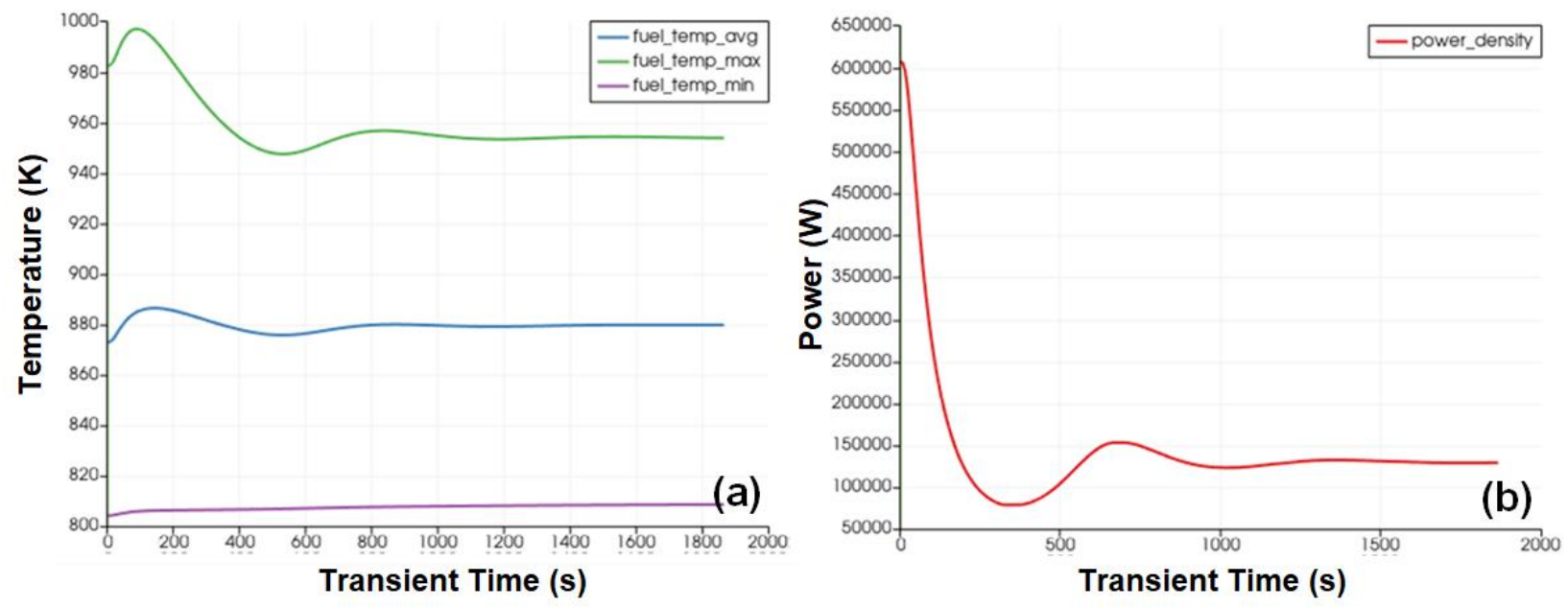

Figure 7-7. Time evolution of fuel temperature and power during the load following power transient. 

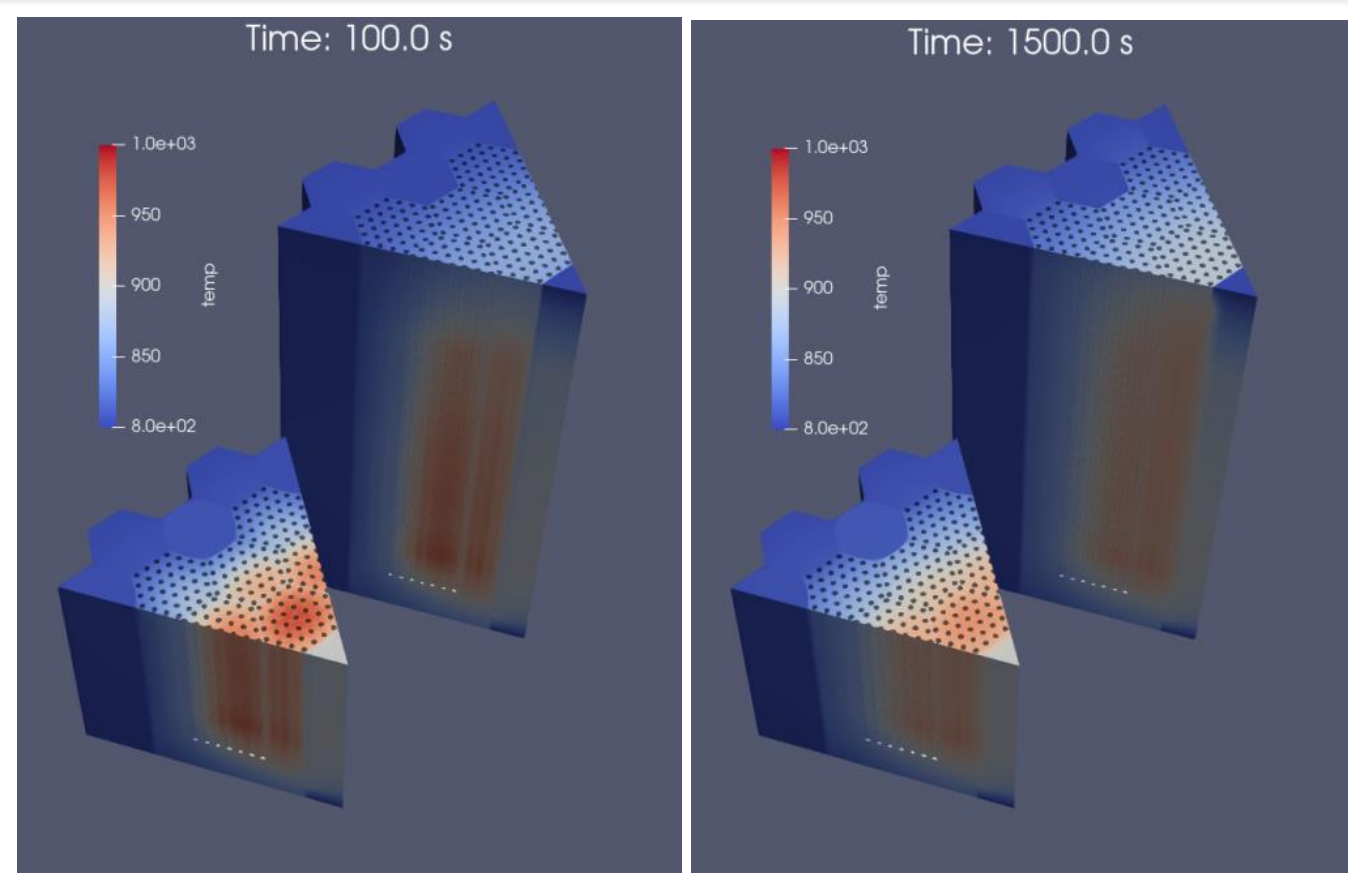

Figure 7-8. The snapshots of temperature profile around the maximum temperature (left) and new-steady-state (right) time point after the load following power transient.

\subsection{Heat-pipe cascading failure (full-core)}

Aside from the load following power transient, single heat pipe failure power transient scenario was also simulated using the same Griffin-BISON-Sockeye coupling strategy above-described. Instead of reducing convective heat transfer coefficients of all the 192 heat pipes in Sockeye subapplications, only one heat pipe was artificially shut down for the single heat pipe failure transient simulation (which means that in the real full-core simulation, we would expect 6 heat pipes to fail simultaneously).

In order to show representative and prominent changes during such power transients, the hottest heat pipe (near the middle of the central assembly in the 1/6-core mesh) was selected to be shut down. The one major concern of the single heat pipe failure event is the consequent failures of its adjacent heat pipes as their operating limits are exceeded due to the failure of the first heat pipes. This phenomenon is termed "heat pipe cascading failure". The objective of this section is to demonstrate that the MOOSE MultiApps system is capable of predicting such cascading failure events and supporting designs to prevent the occurrence of such events. Therefore, a series of postprocessors are set up in the Sockeye second-level sub-applications to calculate the real-time heat-pipe operating limits including boiling limit, capillary limit, entrainment limit, sonic limit, viscous limit. These limits are then compared with the heat pipe loading to determine whether the heat pipe fails or not. 


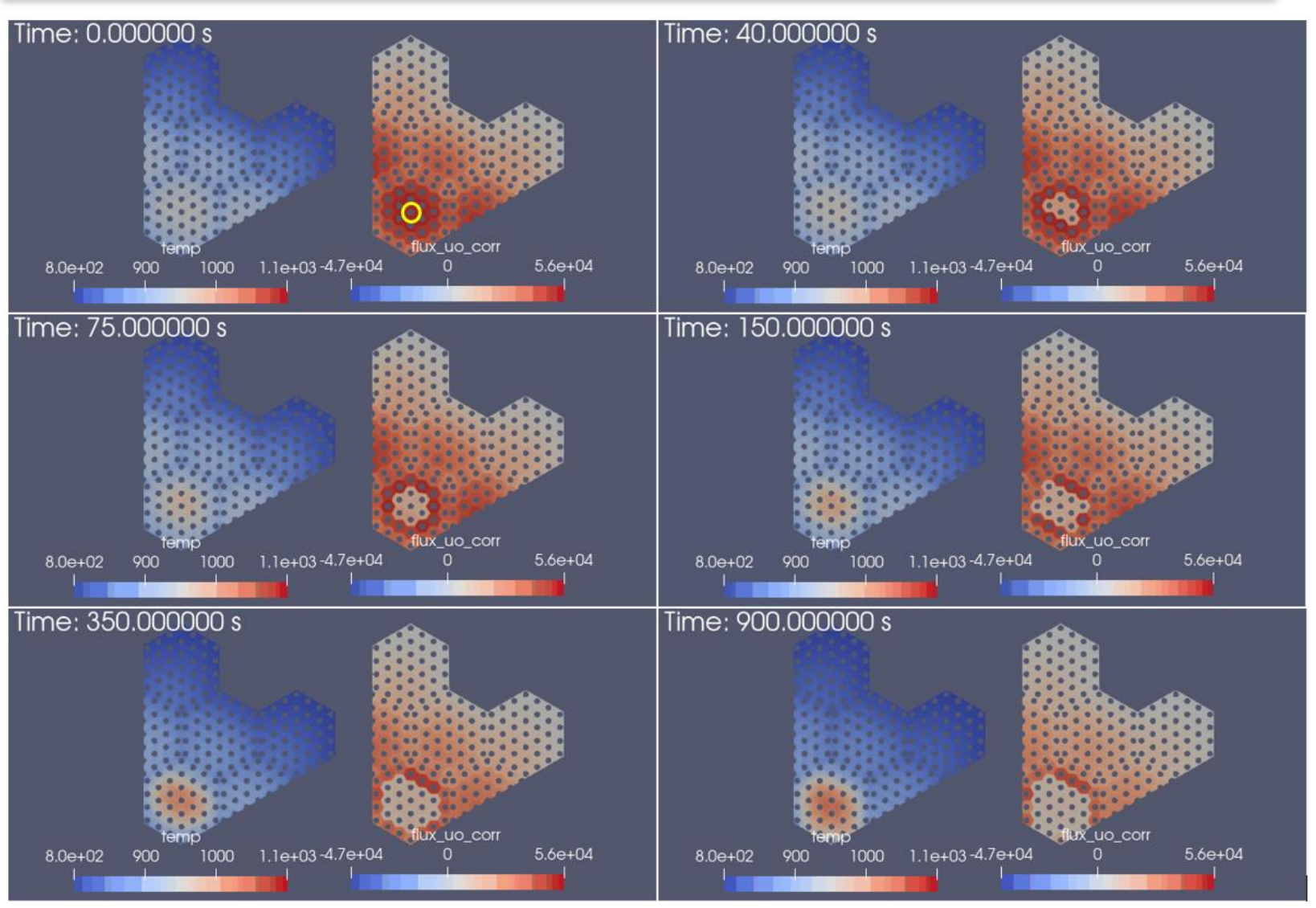

Figure 7-9. Heat pipe cascading failure after a single heat pipe failure (highlighted by a yellow circle) in the 1/6-core operating at $607,000 \mathrm{~W}$.

The single heat pipe failure simulation setting was first used to investigate the $82 \%$-augmented full power (i.e., $607,000 \mathrm{~W}$ for the $1 / 6$ core). A consequent heat pipe cascading failure was predicted by the MultiApps simulation. As shown in Figure 7-9, four adjacent heat pipes fail following the initial single heat pipe failure after 40 seconds. The number of the adjacent failed heat pipes increases to six at 75 seconds, to ten at 150 seconds, to 15 at 350 seconds, and eventually to 20 at 900 seconds. The cascading failure leads to a significant increase in temperature. To be specific, the maximum fuel temperature increases from $\sim 980 \mathrm{~K}$ up to $\sim 1100 \mathrm{~K}$ as the new steady state is reached. More importantly, due to the cascading failure, a large region within the central assembly suffers high temperature. The cascading failure originates from the fact that the operating power was intentionally set close to the heat pipe operating limits. In that case, as one single heat pipe fails, the operating limits of the adjacent heat pipes are exceeded in short time, leading to a cascade event. The operating limit that is exceeded here is usually the entrainment limit.

In order to prevent such cascade event from happening, the designed operating power should be reduced to leave sufficient safety margin so that the operating limits of adjacent heat pipe are not exceeded during a single heat pipe failure event. (An alternative option that may not require reducing the core power would be to increase the operating temperature of the core beyond $900 \mathrm{~K}$, which will provide more margins to operating limit of the heat pipes as shown in Figure 6-1. This option is not further investigated in this report.) A single heat pipe failure simulation using conventional power (i.e., 345,600 $\mathrm{W}$ for the 1/6 core) was performed. As shown in Figure 7-10, the system was predicted by the MOOSE MultiApps simulation to avoid any further heat pipe 
cascading failure event. After 1,000 seconds since the initiation of the single heat pipe failure; all the other heat pipes operate normally throughout the transient scenario. The high temperature caused by the single heat pipe failure event is also localized near the failed heat pipe. That is, according to the MOOSE MultiApps simulation, the HP-MR design does not suffer heat pipe cascading failure due to the failure of a single heat pipe when it operates at the reference power $(\sim 2$ MW th).

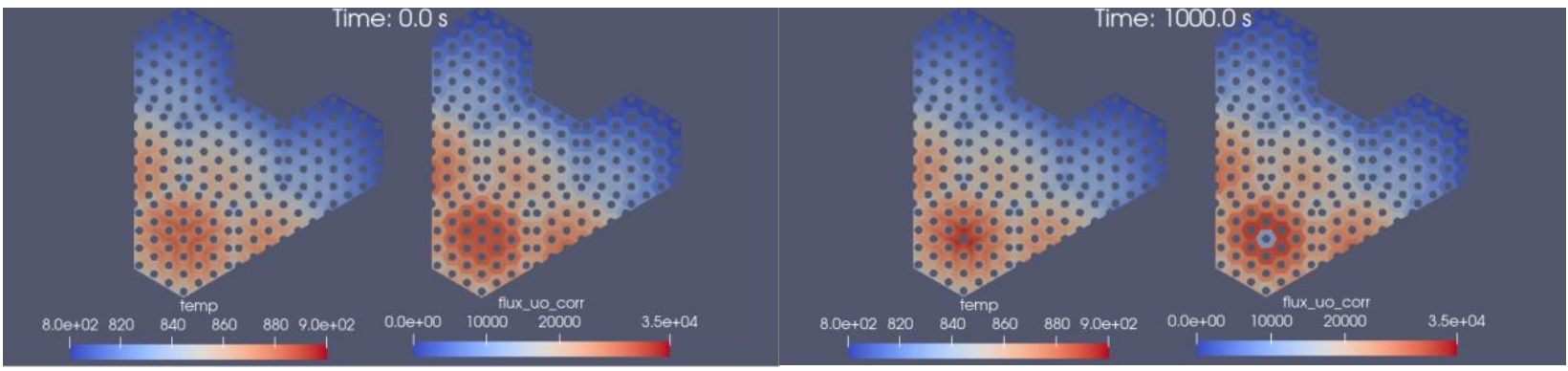

Figure 7-10. Simulation results of the single heat pipe failure in the 1/6-core operating at 345,600 $\mathrm{W}$ showing absence of heat pipe cascading failure.

Hence, it has been demonstrated that the 3D MOOSE MultiApps (Griffin-BISON-Sockeye) simulation approach for 1/6 microreactor core is capable of predicting heat pipe cascading failure events. Therefore, the MOOSE MultiApps framework can be used to support design of an optimized operating power level that provides both high efficiency and sufficient safety margins.

\subsection{Decay heat removal (full-core)}

Decay heat removal was modeled through simplified SAM-MOOSE transient simulations. These simulations were assembled to demonstrate decay heat removal modeling capabilities with SAM and other MOOSE-based codes. The simulation results presented herein are meant to be used as proof-of-concept, as modeling of the physics across the core will be carried out by other MOOSEbased codes. The implementation of some of the tools and approaches used in these simulations have been previously tested in a study by $\mathrm{Hu}$ et al. [10].

In the transient of interest, it is assumed that heat removal through the heat pipes is lost, and that decay heat is entirely removed through a simplified air Reactor Cavity Cooling System (RCCS). RCCS systems are designed to operate passively and remove decay heat from the reactor vessel during accident scenarios. These systems take advantage of natural circulation to induce air flow to remove decay heat. Typically, air RCCS designs consist of inlets and outlets exposed to ambient air, and are vertically separated by a tall chimney to stablish a pressure difference. Through this design, cold ambient air is drawn at the inlets and hot air is exhausted at the outlets of chimneys, which induce natural circulation. Since a specific RCCS configuration for this design is not available, a simplified model is used instead.

For this demonstration case, MOOSE is used to model the heat conduction in the core, and SAM is utilized to model the thermal radiation and heat removal by the simplified air RCCS model. A schematic of such model is shown in Figure 7-11 (applied in this schematic on a simple core geometry). The SAM model includes a RCCS wall which is heated by the reactor vessel wall through radiative heat transfer, and an air channel which removes heat through convection. In this simplified RCCS model, a constant velocity and temperature boundary condition is imposed at the 
inlet of the air fluid component, while a pressure outlet with ambient temperature conditions are imposed at the outlet.
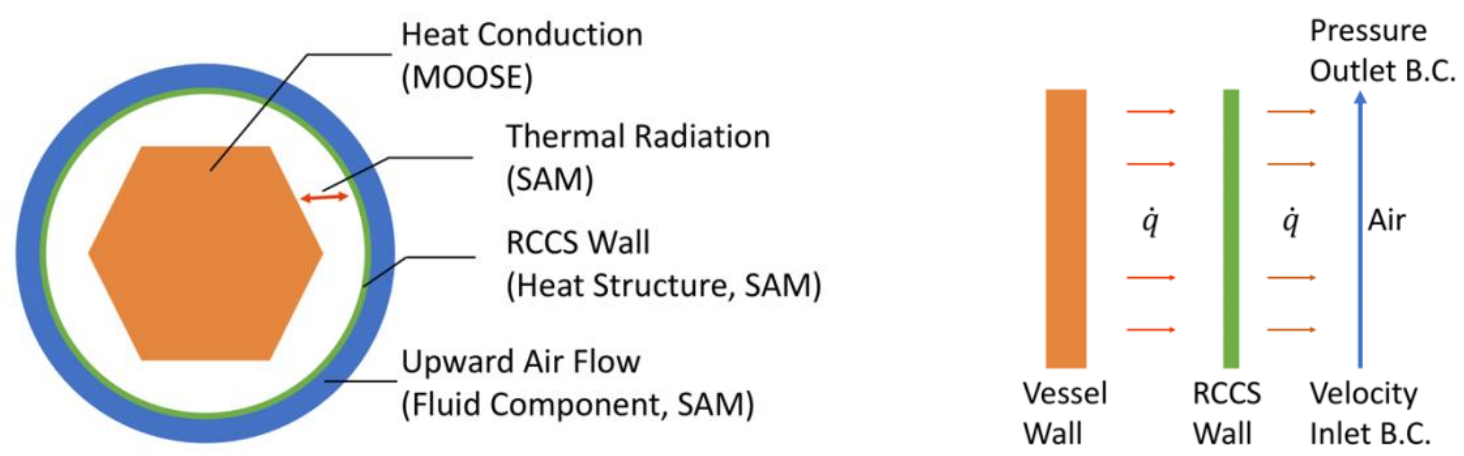

Figure 7-11. Schematic of the simplified RCCS model applied to a simple hexagonal block.

These simulations use the native MOOSE MultiApp and Transfer system to exchange information between the SAM and MOOSE models. Through the MultiApp system, the simulation is divided into three parts: a MOOSE main application and two sub-applications. The main application contains information about the core and the heat conduction equations using MOOSE. The first sub-application is used to transfer the initial conditions obtained from a steady state Griffin-Bison simulation at the first time step, and the second sub-application is used to model the RCCS using SAM throughout the transient. The initial temperature and power distributions of the core $(607 \mathrm{~kW}$ for 1/6 core) used are shown in Figure 7-12. The initialization of these two variables is executed via the nearest node transfer through the MultiApp system. Once the initialization is complete, these distributions are used as the first time step in the transient simulations.
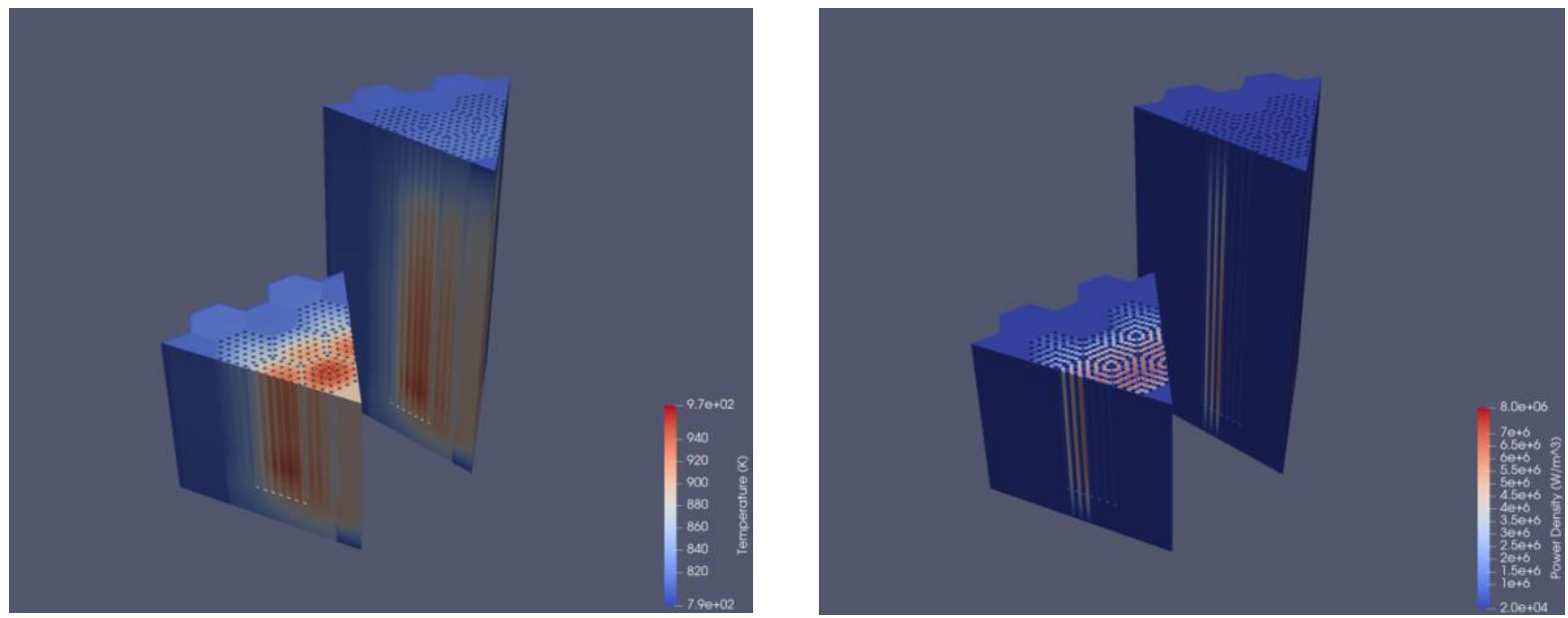

Figure 7-12. Initial temperature distribution (left) and power distribution (right) of the 1/6-core.

Decay heat modeling is incorporated into the main application through the AuxKernel system, which allows for the explicit definition of the decay heat curve as a function. The decay heat curves implemented into our models are shown in Figure 7-13. These include the Wigner-Way correlation and the ANS-94 standard for decay heat, where the latter is used for the analysis in this section since it provides more conservative results. The decay heat is then modeled by applying the decay 
heat profile to the initial power distribution from the first timestep, thus following the pattern of the ANS-94 curve.

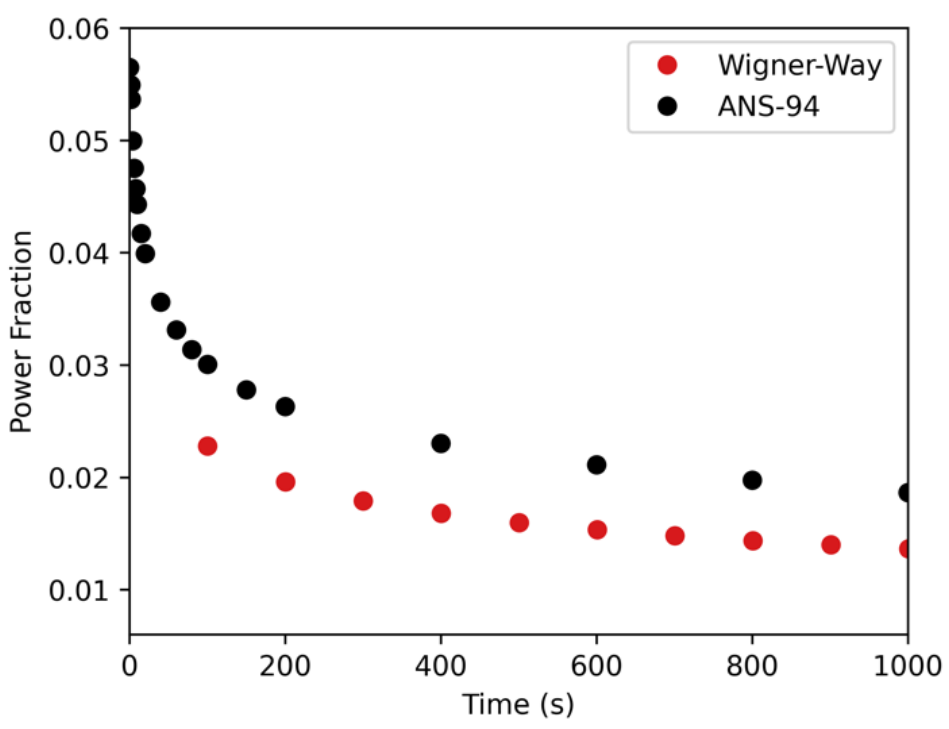

Figure 7-13. Wigner-Way and ANS-94 decay heat curves.

With these implementations, a transient simulation is executed to demonstrate heat removal modeling capabilities with MOOSE and SAM. The simulation is first run for 20 seconds before heat removal by the RCCS is started. Once the power and temperature distributions are initialized, the only heat generated within the core is due to decay heat. The power density distributions at 10 seconds and 500 seconds into the transient are shown in Figure 7-14. Initially, the power density distribution drops to approximately $5.6 \%$ of the initial power and then decays over time following the ANS-94 curve.

Simultaneously, as the decay heat decreases over time, heat is removed via the RCCS. The temperature distributions at two instances are shown in Figure 7-15. Over the span of 500 seconds, the temperature distribution across the core diffuses and decreases over time. It is worth noting that the rate at which heat is removed by the RCCS is a function of the speed at which air travels in the RCCS domain; that is, with higher velocity, more heat can be removed. However, due to the simplification of the RCCS, a fixed velocity of $1 \mathrm{~m} / \mathrm{s}$ and constant air temperature of $300 \mathrm{~K}$ at the inlet is used. 

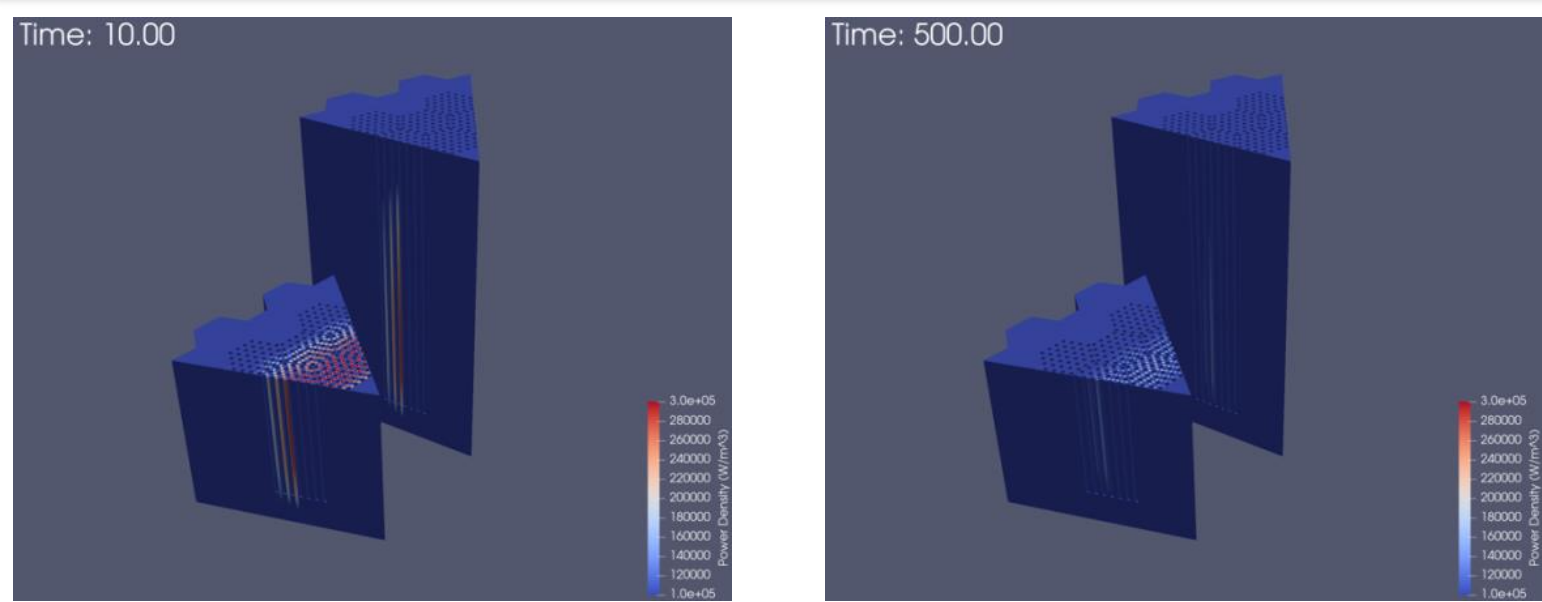

Figure 7-14. Power density distribution at two instances during the simulated transient.
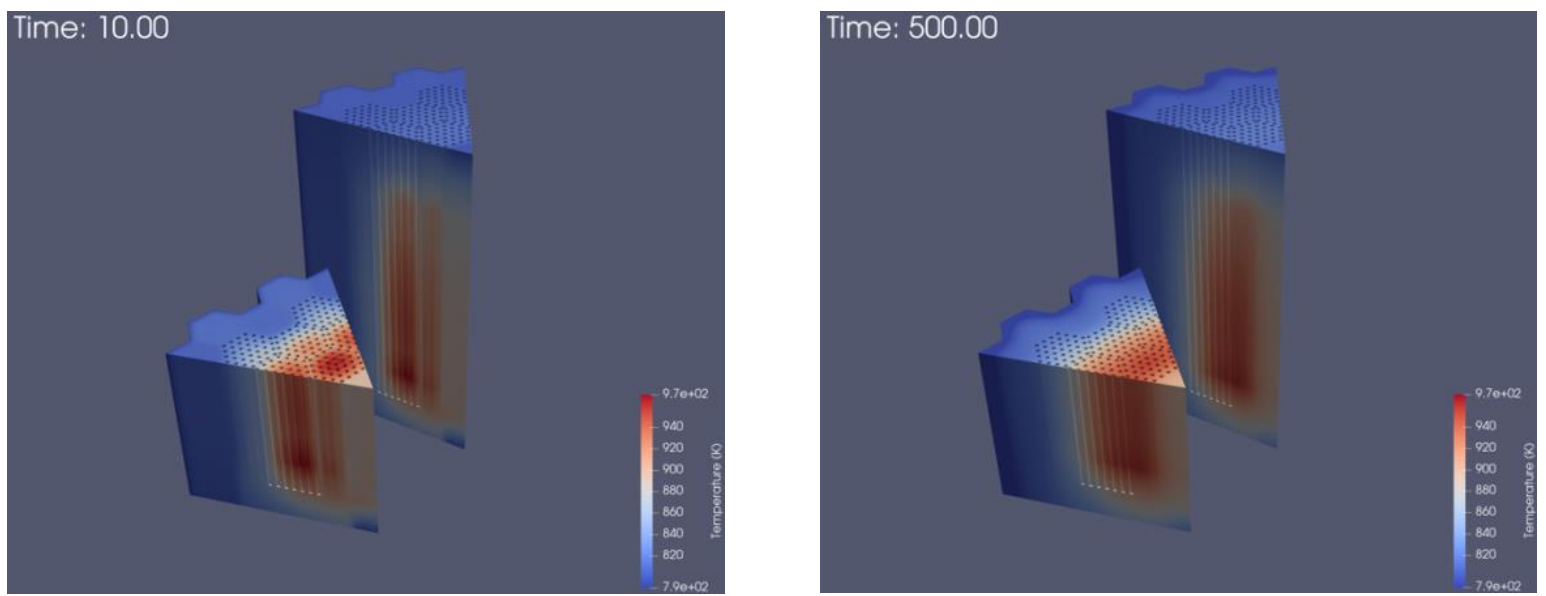

Figure 7-15. Temperature distribution at two instances during the simulated transient.

To better illustrate the decrease in temperature in the core, the maximum fuel temperature during the transient is plotted and provided in Figure 7-16. Here, a temperature increase in the maximum fuel temperature is observed because the initial power distribution is assumed to be constant for the first 20 seconds, and additionally, no heat is removed by the RCCS during this time. At the initial time steps of the transient $(t=0)$ only decay heat is present and heat removal by the RCCS begins. As a result, a decrease in the maximum fuel temperature is exhibited during the first 500 seconds of the simulation. Through this simplified simulation, the level of modeling capabilities of these MOOSE-SAM simulation for decay heat removal via RCCS in micro-reactor designs has been demonstrated. 


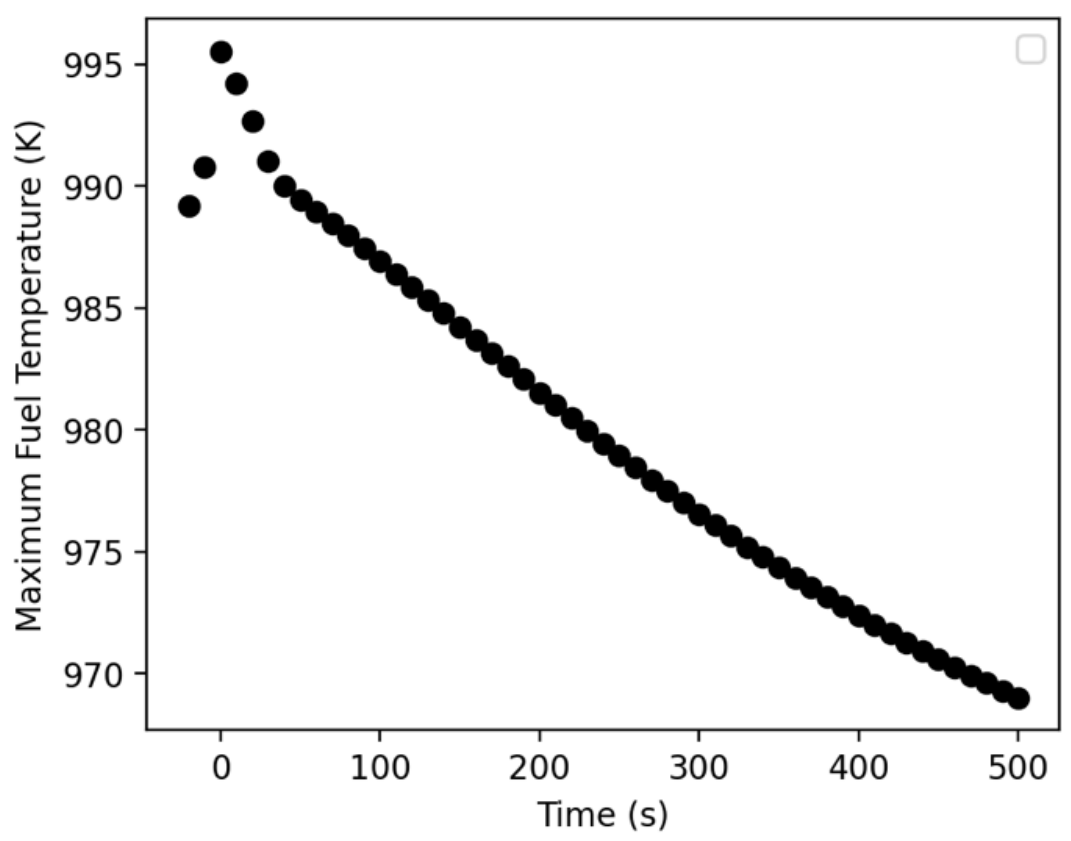

Figure 7-16. Maximum fuel temperature during transient. 


\section{Summary and Conclusions}

The NEAMS Application Drivers Technical Area has been tasked with demonstrating the ability of the NEAMS tools to perform high-fidelity and multiphysics simulations on a representative micro-reactor problem of interest to the U.S. industry. This project focused on training a diverse team of experts in their fields (neutronics, thermal-hydraulics, and fuel thermomechanical analyses) on the NEAMS codes and gathering user feedback in the first half of FY21. The second half of FY22 involved applying these tools to micro-reactor analyses. The work discussed in this report focused on modeling the Argonne-designed HP-MR, which is a TRISO-fueled heat pipe microreactor design developed as a modeling experiment using coupled Griffin, Sockeye, SAM, and BISON codes for high-fidelity multiphysics transient simulations.

Our analysis provides preliminary confirmation of the feasibility of applying the NEAMS codes to model load following transients and accident scenarios. Detailed demonstration was performed on a simple unit-cell model to perform transient simulations, while verifying the accuracy of the multiphysics solution obtained with the NEAMS codes. Results on the full core focused on showcasing the multiphysics workflow with future work planned on verification of the results.

On this unit-cell model, the Griffin diffusion and SPH method has been applied to improve the accuracy of the reactor physics results obtained from the diffusion-only calculation. With this method, not only is the fast convergence rate of the diffusion method preserved in multiphysics simulations, but the axial power distributions and reactivity temperature feedback effects are all calculated accurately. The $S_{N}$ methods have also been applied successfully, but further computational performance improvement is needed to enable application to unit-cell and full-core multiphysics modeling problems. Verification of the Griffin cross-section interpolation methodology used in multiphysics simulations was completed. This work demonstrated that Griffin accurately interpolates cross-sections based on two varying parameters such as the fuel temperature, moderator temperature, and hydrogen content in the moderator. But this analysis showed that finer grids in the cross section lookup tables are also necessary for accurately modeling the temperature feedback effects. Multiphysics load-following transients were completed by coupling Griffin, BISON, and Sockeye through the MultiApp approach. This analysis showcased the impact of these different model improvements on the accuracy of the transient simulations.

Based on the success of the unit cell simulation, the multiphysics simulations were expanded to the full-core geometry. At this time, the Griffin diffusion method (without SPH correction) was used in the full-core multiphysics simulations to demonstrate the workflow for transient simulations. BISON's thermal-mechanical modeling capability coupled with MOOSE's heat conduction and elastic mechanics models was demonstrated on the full-core simulation, while only the thermalmechanical model was used for transients analysis. The effective conduction model of Sockeye was used to model heat transfer through the heat-pipe in our multiphysics simulations, supported by estimates of the thermal conductivity of the vapor core generated with the two-phase models. The multiphysics (BISON/Sockeye) models developed in this work were made available through the NRIC Virtual Test Bed (VTB) repository [35] to reduce the learning curve of future NEAMS industry users. Both unit-cell and full-core load following transients display the self-regulation capability of the HP-MR where a drop in heat-removal rates from the condenser-side of the heatpipe was quickly followed by a power reduction with less than $50 \mathrm{~K}$ of peak fuel temperature variations. 
Aside from the load following power transient, a single heat pipe failure power transient scenario was also simulated, demonstrating that the multiphysics simulation approach coupling Griffin/BISON/Sockeye is capable of predicting heat pipe cascade failure events. The last multiphysics transients modeled considered decay heat removal simulation using SAM/MOOSE coupling through the MultiApp approach to model heat removal from the reactor vessel through the RCCS system during accident scenarios. Finally, a side study was completed to experience the capability of BISON for performing the TRISO fuel particle analysis for microreactor applications.

Therefore, the MOOSE MultiApps framework and NEAMS codes can be used to support some design activities and safety analyses for heat-pipe micro-reactors. Several ongoing improvements in the NEAMS codes will enable further improved modeling of the dynamics modeling of microreactors. Improved Griffin solution of our full-core model is required and will be assessed using $\mathrm{S}_{\mathrm{N}}$ methods or the Diffusion with SPH approach. To reduce computational cost of neutronic simulations during multiphysics transients, a point kinetics model within Griffin or SAM can be utilized. Accurate load-following modeling of such thermal-spectrum micro-reactors should include a depletion model to account for Xenon poisoning. The SWIFT code under development at LANL can be used to model hydride metal moderator capability to retain hydrogen throughout temperature transients. Finally, by leveraging the experience and capability gained in this project, the NEAMS codes should be applied to model the KRUSTY test microreactor to provide experimental validation of multiphysics simulations. 


\section{REFERENCES}

1. https://www.energy.gov/ne/articles/what-nuclear-microreactor

2. C. R. Stanek, Overview of DOE-NE NEAMS Program. United States: N. p., 2019. Web. doi:10.2172/1501761.

3. G. Hu et al., "Multiphysics Simulations of Heat Pipe Micro Reactor," United States: N. p., 2019. Web. doi:10.2172/1569948.

4. C. H. Lee et al. "Micro Reactor Simulation Using the PROTEUS Suite in FY19," United States: N. p., 2019. Web. doi:10.2172/1571248.

5. C. Matthews et al. "Evaluation of the MOOSE Tool-set for Analysis of ThermoMechanical-Neutronics coupling in Micro-Reactors" Los Alamos National Laboratory, LAUR-19-31443.

6. C. H. Lee, J. Ortensi, et al., "Griffin Software Development Plan," ANL/NSE-21/23, INL/EXT-21-63185, Argonne National Laboratory and Idaho National Laboratory, June 2021.

7. R. L. Williamson, et al. "Multidimensional multiphysics simulation of nuclear fuel behavior," J Nucl Mater, 423, 149 (2012)

8. J. E. Hansel et al., "Sockeye Theory Manual," INL/EXT-19-54395, March 2020.

9. Joshua E. Hansel, Ray A. Berry, David Andrs, Matthias S. Kunick, Richard C. Martineau (2021). "Sockeye: A One-Dimensional, Two-Phase, Compressible Flow Heat Pipe Application", Nuclear Technology, 207(7), p. 1096-1117.

https://doi.org/10.1080/00295450.2020.1861879

10. R. Hu et al., SAM Theory Manual, Argonne National Laboratory, ANL/NE-17/4 Rev. 1, February 2021.

11. C. Matthews, A. Shivprasad, M. Cooper, "Metal Hydride Simulations Using SWIFT," July 2021, LA-UR-21-27538.

12. C.J. Permann, et al., "MOOSE: Enabling massively parallel multiphysics simulation", SoftwareX 11 (2020) 100430.

13. D. R. Gaston, C.J. Permann, J.W. Peterson, A.E. Slaughter, D. Andrs, Y. Wayng, M.P. Short, D.M. Pere, M.R. Tonks, J. Ortensi, L. Zou, and R.C. Martineau, "Physics-based multiscale coupling for full core nuclear reactor simulation", Annals of Nuclear Energy, vol 84, pp. 45-54, 2015.

14. N. Stauff, K. Mo, Z. Mei, Y. S. Jung, J. Thomas, L. Ibarra, D. Grabaskas, C. H. Lee, C. Matthews. "Demonstration of Modeling and Simulation Capability for Micro-Reactors," ANL/NEAMS-20/1, Aug. 28, 2020.

15. Derek Gaston, Chris Newman, Glen Hansen, Damien Lebrun-Grandié, "MOOSE: A parallel computational framework for coupled systems of nonlinear equations", Nuclear Engineering and Design, Volume 239, Issue 10, 2009, Pages 1768-1778, https://doi.org/10.1016/j.nucengdes.2009.05.021. 
16. Y. Wang, "Nonlinear Diffusion Acceleration for the Multigroup Transport Equation Discretized with SN and Continuous FEM with Rattlesnake". In: Proceedings to the International Conference on Mathematics, Computational Methods \& Reactor Physics (M\&C 2013). Sun Valley, Idaho, USA, May 2013

17. Nicolas E. Stauff, Kun Mo, Yan Cao, Justin W. Thomas, Yinbin Miao, Changho Lee, Christopher Matthews, Bo Feng, "Preliminary Applications of NEAMS Codes for Multiphysics Modeling of a Heat Pipe Microreactor," proceedings of ANS Annual 2021 meeting, June (2021).

18. R.L. Williamson, et al. "Multidimensional multiphysics simulation of nuclear fuel behavior," J Nucl Mater, 423, 149 (2012)

19. Richard L. Williamson, Jason D. Hales, Stephen R. Novascone, Giovanni Pastore, Kyle A. Gamble, Benjamin W. Spencer, Wen Jiang, Stephanie A. Pitts, Albert Casagranda, Daniel Schwen, Adam X. Zabriskie, Aysenur Toptan, Russell Gardner, Christoper Matthews, Wenfeng Liu \& Hailong Chen (2021) BISON: A Flexible Code for Advanced Simulation of the Performance of Multiple Nuclear Fuel Forms, Nuclear Technology, 207:7, 954-980

20. https://cubit.sandia.gov

21. J. Leppanen, "Serpent - a Continuous-energy Monte Carlo Reactor Physics Burnup Calculation Code," User's Manual, June 18 (2015).

22. Y. Wang, J. Ortensi, V. Laboure, J. Harter, O. Calvin, W. Dawn, "ISOXML - The Multigroup Cross Section Library Tools," (2020)

23. H. Zhang, D. Andrs, J. Hansel, L. Zou, R. Berry, R. Martineau, "RELAP-7 User's Guide”, INL/EXT-14-33977-Rev1, March 2018, doi 10.2172/1466669.

24. M.B. Chadwick, M. Herman, P. Obložinský, M.E. Dunn, Y. Danon, A.C. Kahler, D.L. Smith, B. Pritychenko, G. Arbanas, R. Arcilla, R. Brewer, D.A. Brown, R. Capote, A.D. Carlson, Y.S. Cho, H. Derrien, K. Guber, G.M. Hale, S. Hoblit, S. Holloway, T.D. Johnson,. "ENDF/B-VII.1 Nuclear Data for Science and Technology: Cross Sections, Covariances, Fission Product Yields and Decay Data." Nuclear Data Sheets 112.12 (2011): 2887-2996.

25. Javier Ortensi, Yaqi Wang, Alexandre Laurier, Sebastian Schunert, Alain Hébert, Mark DeHart, "A Newton solution for the Superhomogenization method: The PJFNK-SPH," Annals of Nuclear Energy, 111, 2018, Pages 579-594, https://doi.org/10.1016/j.anucene.2017.09.027.

26. J.D. Hales, R.L. Williamson, S.R. Novascone, D.M. Perez, B.W. Spencer, G. Pastore, Multidimensional multiphysics simulation of TRISO particle fuel, Journal of Nuclear Materials 443 (2013) 531-543

27. G.K. Miller, D.A. Petti, J.T. Maki, D.L. Knudson, and and W.F. Skerjanc. PARFUME Theory and Model Basis Report. Report INL/EXT-08-14497 (Rev.1), Idaho National Laboratory, September 2018

28. https://mooseframework.inl.gov/BISON/tutorials/advanced_fuels/triso_tutorial/triso_parfu me_overview.html 
29. A. Hebert and P. Benoist, "A Consistent Technique for the Global Homogenization of a Pressurized Water Reactor Assembly," Nucl. Sci. Eng., 109, 360 (1991); http://dx.doi. org/10.13182/NSE109-360.

30. A. Hebert and G. Mathonniere, "Development of a Third-Generation Superhomogénéisation Method for the Homogenization of a Pressurized Water Reactor Assembly,” Nucl. Sci. Eng., 115, 129 (1993); http://dx.doi.org/10.13182/ NSE115-129.

31. T. Kozlowski, "Spatial Homogenization Methods for Pin-by-Pin Neutron Transport Calculations," PhD Thesis, Purdue University (2005).

32. A. Yamamoto et al., "Improvement of the SPH Method for Multi-Assembly Calculation," Proc. PHYSOR 2004, Chicago, Illinois, April 25-29, 2004, American Nuclear Society (2004).

33. Bin Zhang, Hongchun Wu, Yunzhao Li, Liangzhi Cao \& Wei Shen (2017) Evaluation of Pin-Cell Homogenization Techniques for PWR Pin-by-Pin Calculation, Nuclear Science and Engineering, 186:2, 134-146, DOI: 10.1080/00295639.2016.1273018

34. Ortensi, J. et al, A Newton solution for the Superhomogenization method: The PJFNKSPH. Annals of Nuclear Energy 111 (Jan. 2018), pp. 579-594.

35. Abdalla Abou-Jaoude, Guillaume Giudicelli, Derek Gaston, Paolo Balestra, Nicolas Martin, and Cody Permann, Bo Feng, Jun Fang, April Novak, Nicolas Stauff, Thanh Hua, Dillon Shaver, and Ling Zou "Overview of Advanced Reactor Simulation Capabilities to Support Demonstrations,” June 2021, INL/EXT-21-63162. 


\section{APPENDIX 1: SPH Factors in Griffin}

\subsection{SPH theory}

The superhomogenization method (SPH) is a cross section correction method which was developed to reduce errors originated from the homogenization process [29, 30]. It is aimed to produce integral reaction rates from the low-order calculations with the same accuracy of those obtained from the higher-fidelity calculation. For instance, for PWR, the SPH factors have been applied to reduce errors in the PWR pin-cell homogenization [31, 32, 33]. Traditionally, a two-step calculation scheme was implemented which includes 1) the calculation of the SPH factors in the lattice neutrontransport calculations and 2) the application of SPH factors to full core diffusion or other low-order calculations.

In Griffin, the SPH method is one of the equivalence methods implemented to reduce errors stem from the homogenization [34] for multiphysics transient simulations. In particular, the SPH factors are generated at each grid states of the multiphysics cross section look-up table. At each grid state, the SPH factors are defined to preserve the reaction rates in an equivalence region " $\mathrm{m}$ " at " $\mathrm{g}$ " energy group using reference results from high-fidelity neutron transport calculations (normally the corresponding Monte Carlo model of the core for generating cross sections):

$$
\mu_{m, g}=\frac{\Sigma_{m, g} \phi_{m, g}^{r e f}}{\Sigma_{m, g} \phi_{m, g}}=\frac{\phi_{m, g}^{r e f}}{\phi_{m, g}}
$$

where $\phi_{m, g}^{r e f}$ is the region-averaged neutron flux obtained from the "reference" calculation, and $\phi_{m, g}$ is the region-averaged neutron flux obtained from the lower order neutron transport equations modified with the SPH factors. For instance, for diffusion and SPH method, $\phi_{m, g}$ is obtained from the modified diffusion equation as:

$$
-\nabla \cdot \mu_{m, g} D_{m, g} \nabla \frac{\phi_{m, g}}{\mu_{m, g}}+\mu_{m, g} \Sigma_{m, g}^{r} \frac{\phi_{m, g}}{\mu_{m, g}}=\frac{\chi_{g}}{k_{e f f}} \sum_{g^{\prime}=1}^{G} \mu_{m, g^{\prime}} v \sum_{f, m, g^{\prime}} \frac{\phi_{m, g^{\prime}}}{\mu_{m, g^{\prime}}}+\sum_{g \prime \neq g}^{G} \mu_{m, g^{\prime}} \Sigma_{s, m}^{g \leftarrow g} \frac{\phi_{m, g^{\prime}}}{\mu_{m, g^{\prime}}}
$$

Equation (2) is nonlinear and the PJFNK solver can be used to solve the $\mu_{m, g}$ and $\phi_{m, g}$ in Griffin.

The diffusion and SPH method developed in Griffin has been applied in the past to improve the accuracy of the diffusion calculation on a full core micro reactor model with homogenized blocks around each heat pipe for multiphysics coupled simulations [3]. Our numerical models for the microreactor have its main components such as fuel pins, moderator pins represented as separate regions in reactor physics calculations. A heterogeneous core model in the reactor physics calculation enables direct data transfer of the reactor power profile from Griffin to the thermalmechanics module without approximations, but often leads to large errors in neutronics calculations using the Diffusion solver. In this study, the diffusion and SPH method was applied on the 3-D unit cell model for load following simulation. The main purpose of the study is to test if the diffusion and SPH method can improve the accuracy of the diffusion calculation in the multiphysics simulations which are based on a heterogeneous core model. The reference results used to improve the diffusion method are from the Serpent -2 unit cell model which provides fluxes for all the mesh blocks. 


\subsection{SPH method procedure in Griffin and verification of SPH factors for the Unit-Cell model}

For multiphysics simulations, the SPH factors need to be generated at each grid point of the crosssection look-up table. It followed the same procedure as described in the Griffin user manual [6]:

a. An additional equivalence zone map was first setup in the Griffin input for each grid point. The equivalence zones are regions where we decide to preserve the reference regionaveraged reaction rates.

b. The reference region-averaged multigroup neutron fluxes were tallied using the Serpent-2 Monte Carlo model at each equivalence zone for each grid point. They are converted to the ISOXML format for Griffin to read.

c. Griffin reads these reference fluxes and solved the modified diffusion equation to compute SPH factors using the Preconditioned Jacobian-Free Newton Krylov (PJFNK) method.

The reference k-eff is an imported input parameter in Griffin for computing the SPH factors correctly. If the SPH factors are calculated correctly, the k-effs and the zone-averaged fluxes (or reaction rates) obtained from the modified diffusion equation will match the reference as shown in Table A1-1 and Figure A1-1.

Table A1-1. Calculated k-effs of the 3-D unit cell isothermal models from Serpent-2 and from diffusion and SPH method.

\begin{tabular}{|c|c|c|c|c|c|}
\hline & $\mathrm{T}=600 \mathrm{~K}$ & $\mathrm{~T}=700 \mathrm{~K}$ & $\mathrm{~T}=800 \mathrm{~K}$ & $\mathrm{~T}=1000 \mathrm{~K}$ & $\mathrm{~T}=1200 \mathrm{~K}$ \\
\hline Serpent & $\begin{array}{c}0.98056 \\
(4 \mathrm{pcm})\end{array}$ & $\begin{array}{c}0.97927 \\
(4 \mathrm{pcm})\end{array}$ & $\begin{array}{c}0.978114 \\
(4 \mathrm{pcm})\end{array}$ & $\begin{array}{c}0.97560 \\
(4 \mathrm{pcm})\end{array}$ & $\begin{array}{c}0.97260 \\
(4 \mathrm{pcm})\end{array}$ \\
\hline $\begin{array}{c}\text { Diffusion } \\
\text { and SPH }\end{array}$ & 0.950563 & 0.979273 & 0.988109 & 0.975595 & 0.972604 \\
\hline
\end{tabular}




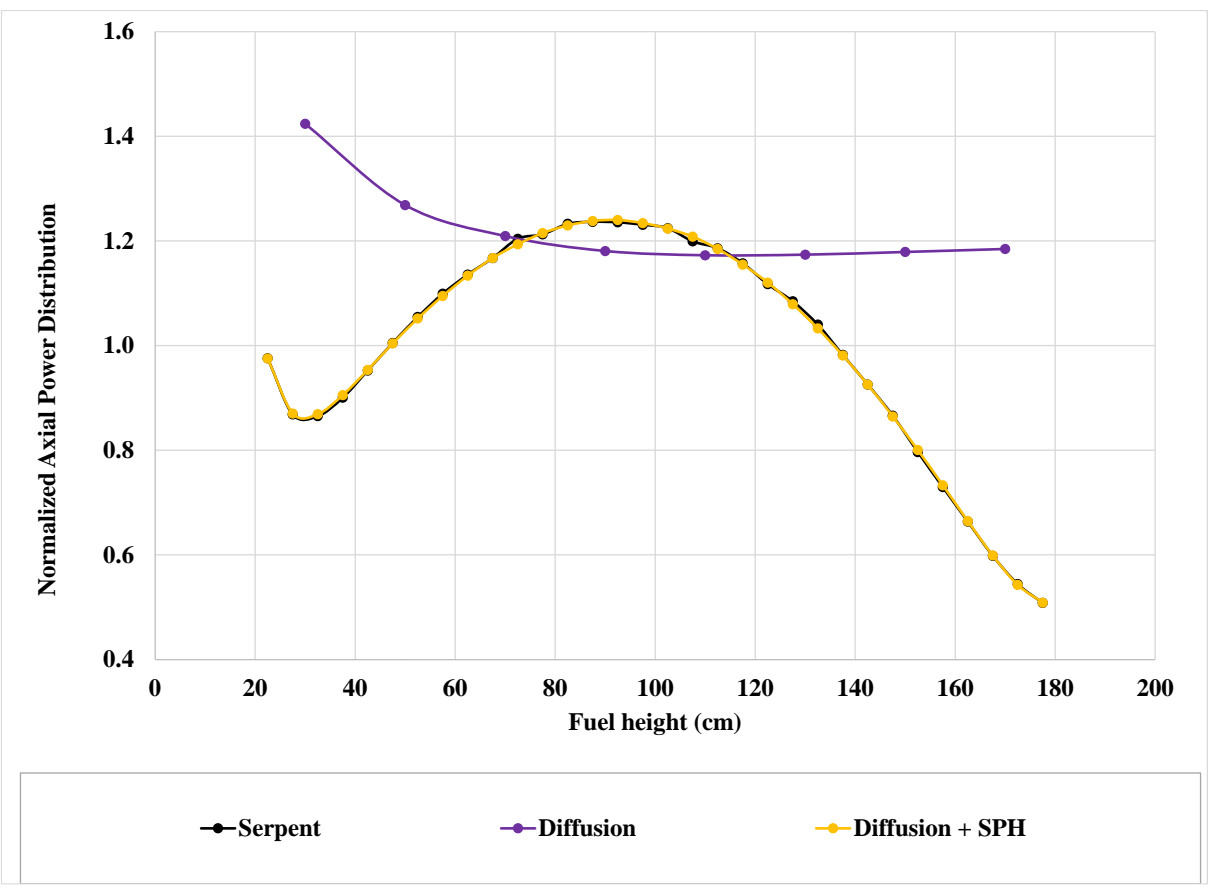

Figure A1-1. Axial power distributions calculated from Serpent-2 model, the diffusion method, and the diffusion and SPH method for the 3-D unit cell model at fuel and moderator temperature at $800 \mathrm{~K}$.

The results shown in Table A1-1 and Figure A1-1 are obtained from a 3-D unit cell model where all the material blocks in the finite element mesh was covered by the equivalence zone map shown in the right plot of Figure A1-2. In radial direction, there is no homogenization and every material region has its own equivalence zone. In the axial direction, a total of 40 equivalence zone was used (every axial layer in the FEM mesh is an equivalence zone). With the SPH factors, the reaction rates obtained from the modified diffusion calculation were preserved in all of the 208 equivalence zones. The axial power distribution within the fuel pins exactly matched the reference result as shown in Figure A1-1. 


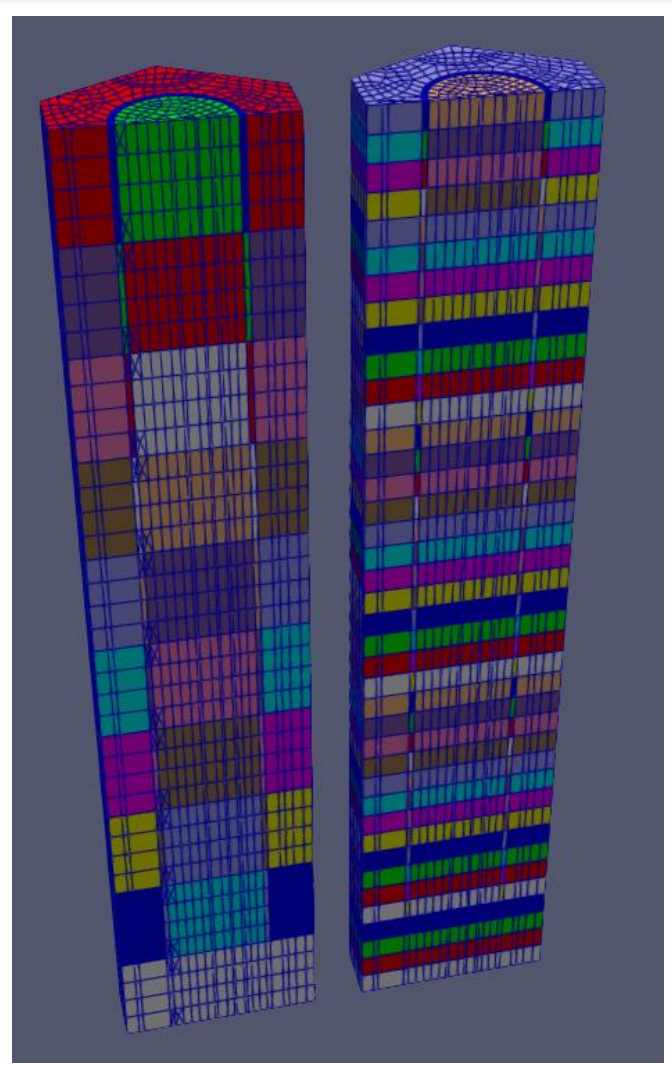

Figure A1-2. SPH equivalence zone map: Left- 10 axial equivalence zone; Right - 40 axial equivalence zone.

For comparison, a second case with only 10 equivalence zone in the axial direction was also tested. The equivalence zone map is shown in the left plot in Figure A1-2, where the radial equivalence zones were the same as in the previous case and less number of zones were used in the axial direction. The SPH factors were calculated to preserve the average reaction rates among every four axial layers. Figure A1-3 compared the axial total neutron flux distributions obtained from the diffusion SPH methods with that from Serpent-2 simulations. The 3-D unit cell model fuel and moderator temperature is $600 \mathrm{~K}$. The fluxes at each axial point were tallied within a $5 \mathrm{~cm}$ axial mesh in the fuel pins if the mesh was in the active fuel region, or in the reflectors if the mesh was in the non-fuel region. The fluxes obtained from Griffin are SPH factors corrected. For the case with 10 axial equivalence zone, the flux was obtained using a user object defined in Griffin. For the case with 40 axial equivalence zone, the flux was directly obtained from the Griffin postprocessor. Clearly, as shown in the figure, the diffusion and SPH method preserved the zoneaveraged reaction rates at the equivalence regions. But for the 10 equivalence zone case, the axial flux distributions were not smoothed. This discontinuous shape is not physical, and propagated to the axial power distributions and temperature distributions in the multiphysics simulations as shown in Figure A1-4, indicating a fine equivalence zone mapping is necessary for accurately modeling the physics in this microreactor. 


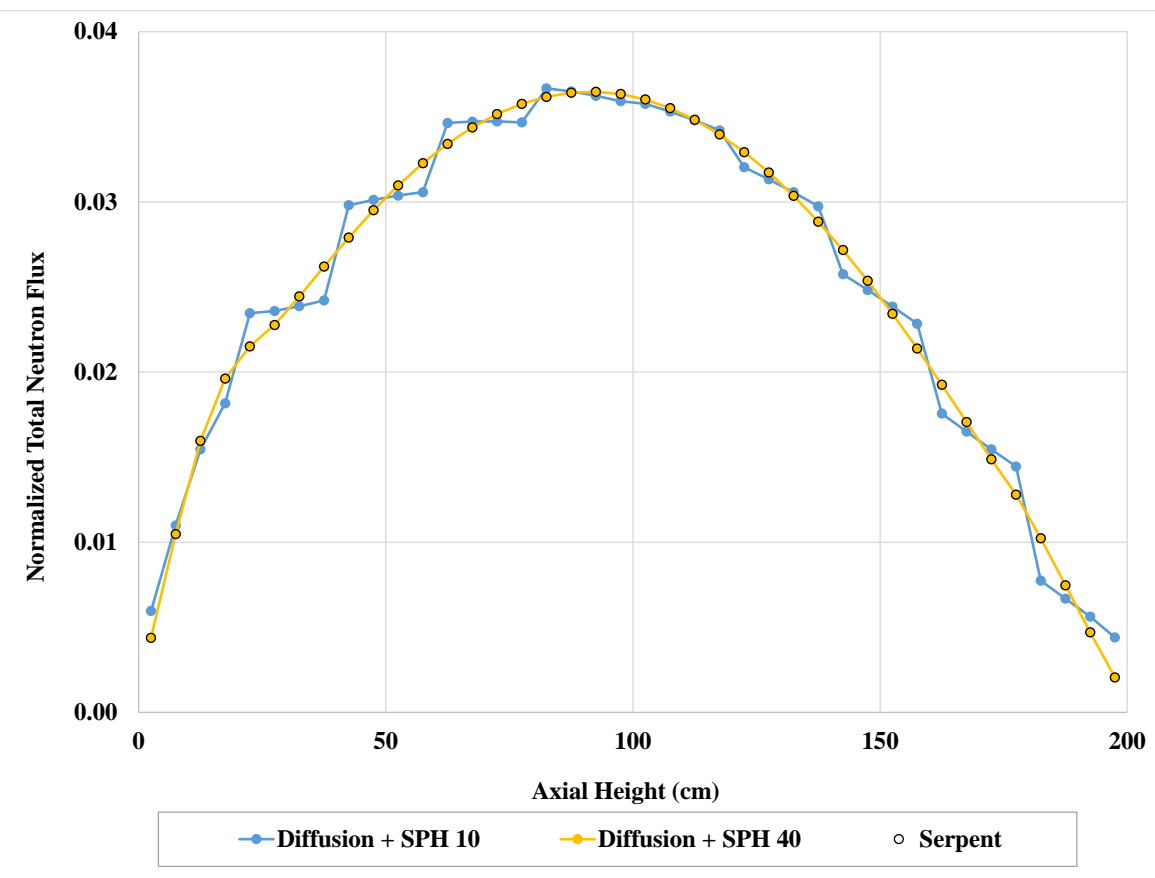

Figure A1-3. Normalized axial total flux distributions calculated from the Serpent-2 model, the diffusion and SPH method with 10 axial equivalence zone, the diffusion and SPH method with 40 axial equivalence zone for the 3-D unit cell model at fuel and moderator temperature at $600 \mathrm{~K}$.

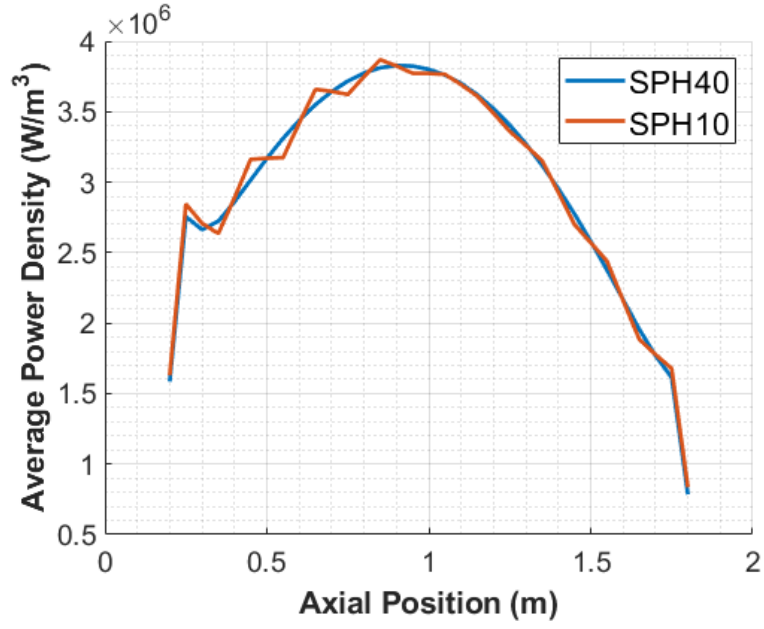

a)

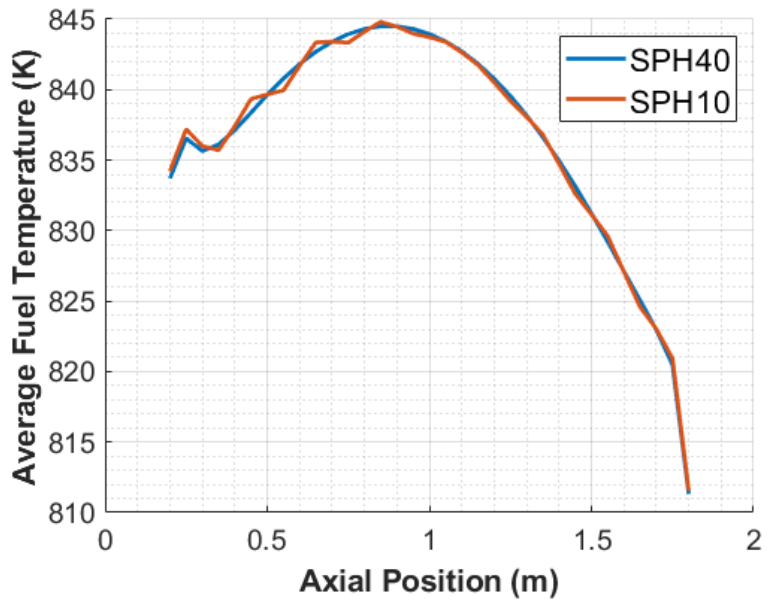

b)

Figure A1-4. Calculated a) axial power density distributions and b) axial averaged fuel temperature distributions in the 3-D unit cell at steady state from the multiphysics simulations with the diffusion and SPH method using 10 axial equivalence zones or 40 axial equivalence zones. 


\subsection{Temperature-dependence of SPH factors}

In multiphysics simulations, the SPH factors for temperatures among the grid points were interpolated between the calculated SPH factors at the temperature grid points. A user utility code was used to convert the SPH factors computed at each equivalence region, each energy group and at each temperature grid point to a lookup table format for the Griffin calculations. Figure A1-5 compared the calculated SPH factors at four energy groups for the five temperature grid points. The SPH factors are those in the reflectors for the non-fuel region and those in the fuel pins in the active fuel region. It shows that the SPH factors all have similar spatial distributions and only slightly different from each other in values at different temperature points, indicating that the piecewise linear interpolation scheme in MOOSE is suited to approximate the actual SPH factors at the middle temperature points (more detailed verification is performed in Appendix 2).

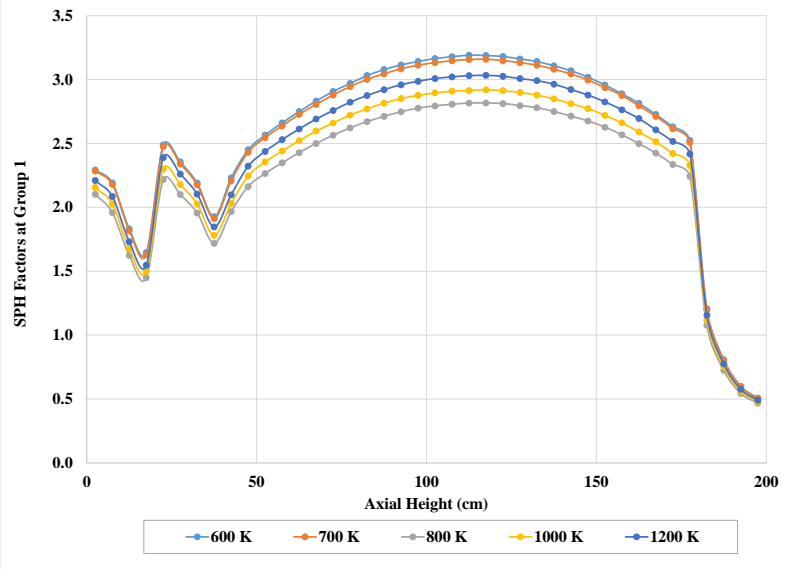

a)

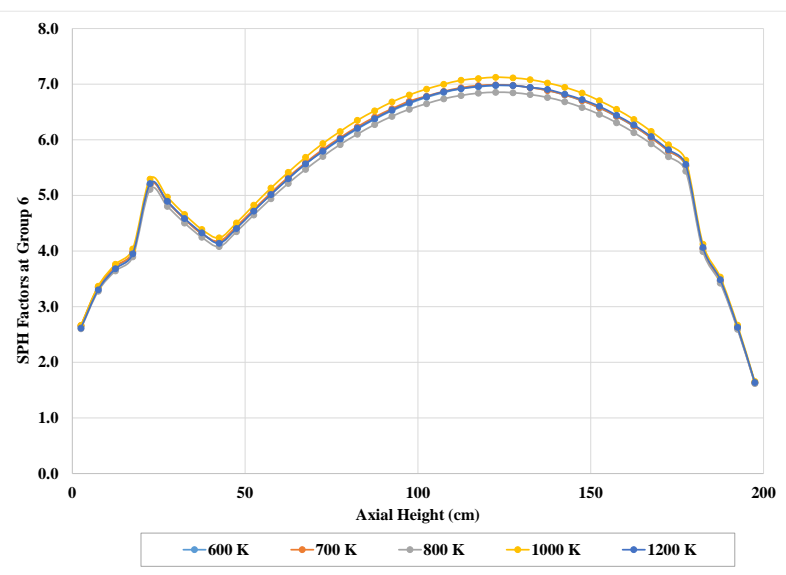

c)

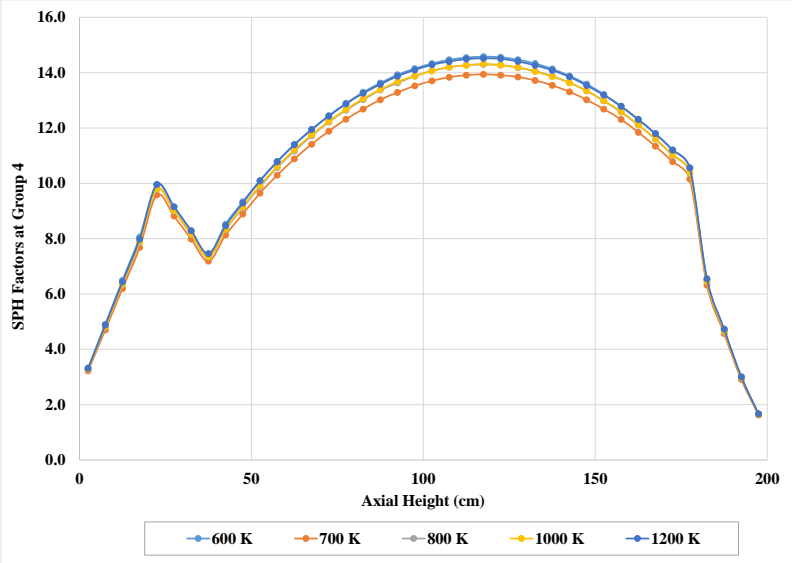

b)

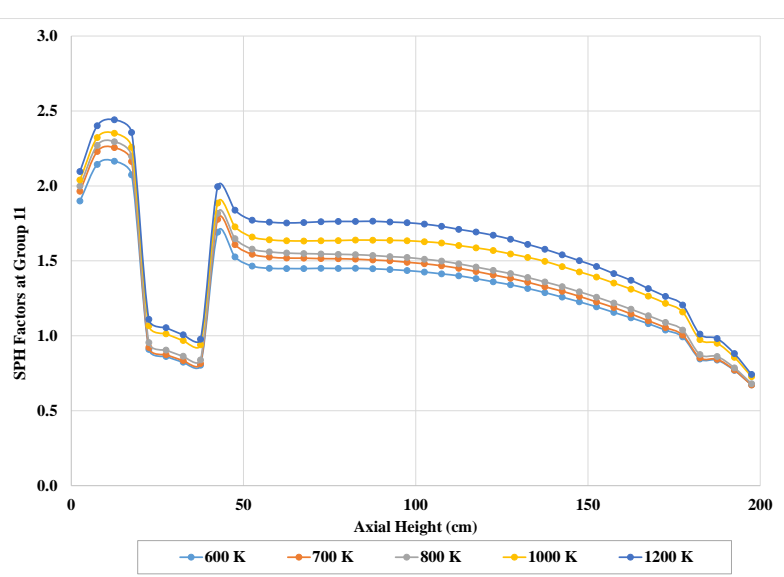

d)

Figure A1-5. Griffin calculated SPH factors for the unit-cell model at temperature grid points of $600 \mathrm{~K}, 700 \mathrm{~K}, 800 \mathrm{~K}, 1000 \mathrm{~K}$ and $1200 \mathrm{~K}$ and energy group: a) [1.0E-5, 8.0E-2] eV; b) [0.625, $1.3] \mathrm{eV}$; ) $[4.0,148.73] \mathrm{eV}$; d) $[1.35,40] \mathrm{MeV}$.

Figure A1-6 plotted the calculated k-effs obtained from the Griffin diffusion and SPH method at the five temperature grid points, and at temperature points among $800 \mathrm{~K}$ and $1000 \mathrm{~K}$ in which the cross sections and SPH factors are interpolated from the look-up tables. It shows that the k-effs are 
matched exactly at the grid points. At those interpolated temperature point, the Griffin calculated k-effs are very close to the expected k-effs from a linear extrapolation of the MC reference value.

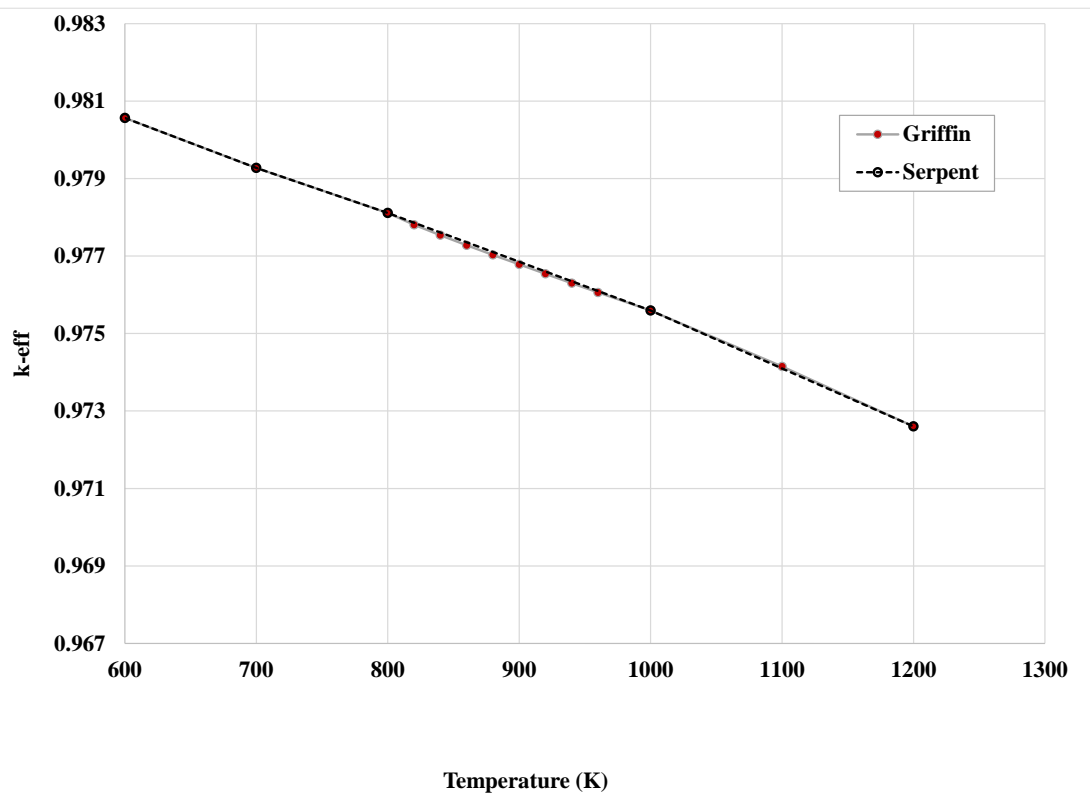

Figure A1-6. Comparison of the calculated k-eff from Griffin using the diffusion and SPH method with interpolated cross sections and SPH factors with Serpent-2 reference result.

\subsection{SPH factors for multiphysics simulations}

A numerical study was also performed to further explore the accuracy of using the interpolated multigroup cross sections and SPH factors in the diffusion and SPH method for multiphysics simulation. First, a fuel and moderator temperature 2-D look-up table was created by only including three grid values at fuel and moderator temperature $\mathrm{T}=600 \mathrm{~K}, 800 \mathrm{~K}$ and 12000 . The multigroup cross sections and the SPH factors for fuel temperatures at $700 \mathrm{~K}$ and $1000 \mathrm{~K}$ were then interpolated from this 2-D look-up table. At these two fuel temperature curves, for those points with moderator temperatures at $700 \mathrm{~K}$ and $1000 \mathrm{~K}$, the cross sections and SPH factors are interpolated among four grid values. For the rest of the points, the interpolation only uses two grid values. Second, the diffusion calculation with SPH method was performed with the interpolated cross sections and SPH factors. For comparison, the cross sections directly obtained from the Speren-2 calculations and SPH factors directly calculated from Griffin PJFNK method were also used to provide the reference results. The differences of k-effs from the two calculations are shown in Table A1-2 (the same table of Table 4-4 in the main report). The differences are small with maximum difference less than 50 $\mathrm{pcm}$. The differences are slightly larger for the four cases where the cross sections and SPH factors were interpolated among 4 grid points.

Table A1-2. Calculated $\Delta \mathrm{k}$-effs from diffusion and SPH method with the direct or interpolated cross sections and SPH factors for the 3-D unit cell model.

\begin{tabular}{|l|l|l|l|l|l|}
\hline Moderator T & $\mathrm{T}=600 \mathrm{~K}$ & $700 \mathrm{~K}$ & $800 \mathrm{~K}$ & $1000 \mathrm{~K}$ & $1200 \mathrm{~K}$ \\
\hline Fuel T=700 K & -0.5 & -1.2 & 6.6 & 48.9 & 4.9 \\
\hline Fuel T=1000 K & -2.3 & -20.8 & -18.1 & 16.0 & -9.0 \\
\hline
\end{tabular}


In addition, the fuel temperature reactivity feedback coefficients $\alpha_{f}$ at each moderator temperature $\mathrm{T}_{\mathrm{i}}$ were calculated using the k-effs obtained at the two fuel temperature $700 \mathrm{~K}$ and $1000 \mathrm{~K}: \alpha_{f}=$ $\left(k_{e f f}^{1000, T_{i}}-k_{e f f}^{700, T_{i}}\right) / 300$, and the moderator temperature feedback coefficients $\alpha_{m}$ were calculated using neighboring temperature points at the same fuel temperature, i.e, for fuel at $700 \mathrm{~K}$ and moderator temperature at $600 \mathrm{~K}: \alpha_{m}=\left(k_{e f f}^{700,700}-k_{e f f}^{700,600}\right) / 100$. As shown in Table A1-3, the fuel temperature coefficients $\alpha_{f}$ obtained from the interpolated cross sections agreed well with that obtained from the direct Serpent- 2 cross sections. The differences are in the range of $0.2 \%$ to $4.7 \%$. The moderator temperature coefficients $\alpha_{m}$ however have larger differences to about a few tens of percent, particularly at those temperature points where the interpolation was performed on the coarser temperature grid. The 2-D look-up tables used in the multiphysics simulations for the 3-D unit-cell model have more temperature grid points than the test look-up table used in this analysis, therefore the temperature feedback effects are better represented in the multiphysics simulations.

Table A1-3 Calculated temperature feedback coefficients ( $\mathrm{pcm} / \mathrm{K}$ ) from Griffin diffusion and SPH method using cross sections directly from Serpent output or using the interpolated cross sections for the 3-D unit cell model.

\begin{tabular}{|c|c|c|c|c|c|c|}
\hline \multicolumn{2}{|c|}{ Moderator T } & $600 \mathrm{~K}$ & $700 \mathrm{~K}$ & $800 \mathrm{~K}$ & $1000 \mathrm{~K}$ & $1200 \mathrm{~K}$ \\
\hline \multicolumn{2}{|c|}{$\alpha_{f}^{\text {direct }}$} & -2.58 & -2.49 & -2.47 & -2.33 & -2.29 \\
\hline \multicolumn{2}{|c|}{$\Delta \alpha_{f}=\alpha_{f}^{\text {interpolate }}-\alpha_{f}^{\text {direct }}$} & -0.005 & -0.065 & -0.082 & -0.11 & -0.046 \\
\hline \multirow{4}{*}{$\Delta \alpha_{f} / \alpha_{f}^{\text {direct }}$} & $0.2 \%$ & $2.6 \%$ & $3.3 \%$ & $4.7 \%$ & $2.0 \%$ \\
\hline \multirow{4}{*}{\begin{tabular}{c} 
Fuel T=700 K \\
Fuel T=1000 \\
\cline { 2 - 7 }
\end{tabular}} & $\alpha_{m}^{\text {direct }}$ & 1.45 & 1.40 & 0.957 & 0.567 & --- \\
\cline { 2 - 7 } & $\Delta \alpha_{m}{ }^{*}$ & 0.007 & -0.078 & -0.21 & 0.22 & --- \\
\cline { 2 - 7 } & $\Delta \alpha_{m} / \alpha_{m}^{\text {direct }}$ & $-0.5 \%$ & $5.6 \%$ & $22.1 \%$ & $-38.8 \%$ & \\
\cline { 2 - 7 } & $\alpha_{m}^{\text {direct }}$ & 1.70 & 1.47 & 1.17 & 0.626 & --- \\
\hline & $\Delta \alpha_{m}{ }^{*}$ & 0.186 & -0.027 & -0.171 & -0.125 & --- \\
\hline
\end{tabular}

$* \Delta \alpha_{m}=\left(\alpha_{m}^{\text {interpolate }}-\alpha_{m}^{\text {direct }}\right)$

Figure A1-7 a) shows that the maximum absolute differences of the axial power densities among all the cases and the maximum difference is found to be less than $5 \%$. Similarly, slightly larger differences are observed for the cases where cross sections are interpolated among 4 grid values than those only interpolated among 2 grid values. The axial power density distributions for the two 
cases with the most difference were compared in Figure A1-7 b) showing that excellent match of the power distributions of the two cases.

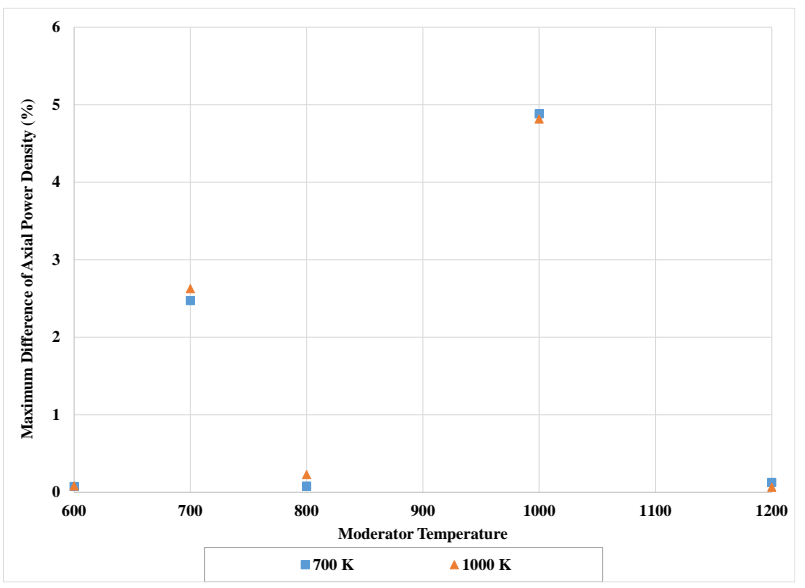

a)

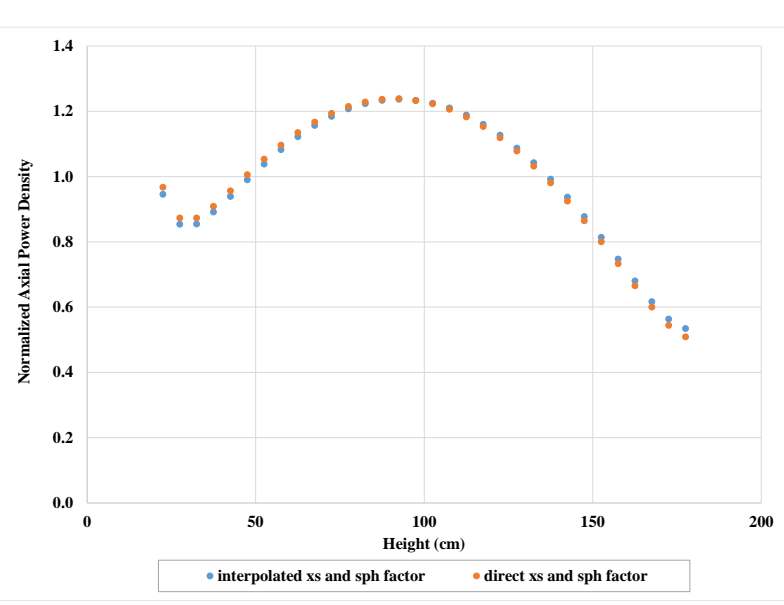

b)

Figure A1-7. a) The maximum differences among the calculated axial power densities using the direct cross sections and SPH factors or using the interpolated cross sections and SPH factors; $b$ ) normalized axial power density distributions for the 3-D unit cell model at $1000 \mathrm{~K}$.

\subsection{Conclusions}

The diffusion method with SPH factors has been successfully applied in microreactor multiphysics simulation with a 3-D unit-cell model. These numerical simulations show that using the SPH factors have greatly improved the accuracy of the diffusion calculated reactor physics results in the transient calculations. Particularly, with the SPH factors being carefully calculated on a fine map within the 3-D unit-cell model, the Monte Carlo reference reactor core eigenvalues and power shapes are preserved from the diffusion calculation at those temperature grid points with the SPH factors explicitly calculated. This assessment on the simple HP-MR unit-cell model also demonstrated that the diffusion and SPH method provides accurate results when the cross sections and SPH factors are interpolated among the grid points. The interpolation of both temperature dependent cross sections and SPH factors can preserve the total power shape in the core well and provide good approximations to the temperature feedback effects. But this analysis showed that finer grids in the cross section lookup tables are also necessary for accurately modeling the temperature feedback effects. In addition, this numerical analysis also shows that the diffusion and SPH method can lead to nonphysical discontinuities of neutron fluxes, power and temperature distributions if the equivalence zone map is not fine enough. More studies on how to generate an appropriate set of SPH factors are needed in future work to apply the method on more complicated reactor models. 


\section{APPENDIX 2: Griffin Cross-sections Generation and Interpolation}

In this section, the detailed cross-section interpolation assessment for the hydrogen dissociation effect will be covered. Two parameters were considered in the assessment, which were the hydrogen density and the temperature of the unit cell model. Meanwhile, two interpolation assessment models are considered. The first interpolation assessment, namely the multigroup crosssection tabulation on $\mathrm{H}$-content in $\mathrm{YHx}$, focuses on the interpolation assessment for the model that has the same physical state throughout the geometry. The second interpolation assessment, namely the heterogeneity effects in multigroup cross-section generation, focuses on the interpolation assessment for the model that has varied physical states throughout the geometry. The methodology and results will be discussed separately for these two topics.

\subsection{Multigroup cross section tabulation on $\mathrm{H}$-content in $\mathrm{YHx}$}

\subsubsection{Assessment procedures}

In MOOSE multiphysics simulation framework, the material properties which often depends on some variables are modeled with piecewise linear functions. The variables are referred as "grid" variables, with the material properties being prepared at the selected grid points to form a look-up table for the multiphysics simulations. For reactor physics calculations performed in the HP-MR microreactor model, the temperature dependence of the multigroup cross sections were modeled by interpolating the prepared cross sections on a two-dimension temperature grid: on grid variable for the fuel temperature in the model and one grid variable for the moderator temperature (as discussed in Section 4.3). Previous discussion in Appendix 1 also showed that a two-dimension look-up table for the SPH factors were created for improving the diffusion calculation in the multiphysics transient simulations. Numerical studies have been performed to demonstrate that the reactor physics can be modeled accurately with cross sections and SPH factor interpolated among the grid values using the piecewise linear functions.

In this section, we also study the multi-group cross-sections dependence on the hydrogen concentrations within the core. In the HP-MR microreactor model, YHx is the moderator used to efficiently slow down neutrons within the core. The hydrogen can disassociate at high-temperature or reduced hydrogen pressure from the YHx matrix, which abridges the deceleration of neutron and makes the flux spectrum harder. To model the impacts of the hydrogen dissociation in the multiphysics simulations, a two-dimensional cross-section lookup table with one dimension for the fuel temperature change and the other dimension for the hydrogen concentration change in the moderator was generated. The material temperatures in the moderator and other regions were assumed to be the same as the fuel temperature.

As depicted in the left plot of Figure A2-1, the MC code Serpent-2 was used to generate the multigroup cross sections for the 2-dimension look up table and providing reference results for later comparison with Griffin. The fuel temperature varied among $600 \mathrm{~K}, 700 \mathrm{~K}, 800 \mathrm{~K}, 1000 \mathrm{~K}, 1200$ $\mathrm{K}$, and $\mathrm{x}$ in $\mathrm{YHx}$ varied from 1.0 to 2.0 with a step of 0.1 , respectively. In total 55 sets of multigroup cross-section sets were generated from the Serpent- 2 calculations. In these simulations, the ENDF/B-VII.1 library was used while the $\mathrm{S}(\mathrm{a}, \mathrm{b})$ libraries for graphite and beryllium reflectors were from ENDF/B-VII.0 library. $\mathrm{YH}_{2} \mathrm{~S}(\mathrm{a}, \mathrm{b})$ was not included in our model since it is not included in the current Serpent-2 build in library. It is understood that there might be an up to $300 \mathrm{pcm}$ impact on the calculated k-effs from the $\mathrm{YH}_{2} \mathrm{~S}(\mathrm{a}, \mathrm{b})$ library. All MC criticality simulations completed 1000 cycles with $10^{5}$ neutrons per cycle. 
Figure A2-1 right plot shows the calculated reference k-eff of the 3-D unit cell ranging from 0.935 to 0.985 . The standard deviation for all the cases is around $7 \mathrm{pcm}$. The k-eff decreases while increasing the fuel temperature as expected due to the large fuel Doppler feedback effects in this reactor. The k-eff also decreases while the hydrogen dissociation process takes place leading to a harder flux spectrum.

The multigroup cross-sections generated from Serpent-2 used an 11-energy group structure (in Table 4-1). These cross-sections form a master set of the XS library which will be used later in the verification study. They were converted to the two-dimension look-up table in the ISOXML format that will be used in Griffin reactor physics calculations and multiphysics transient simulations.
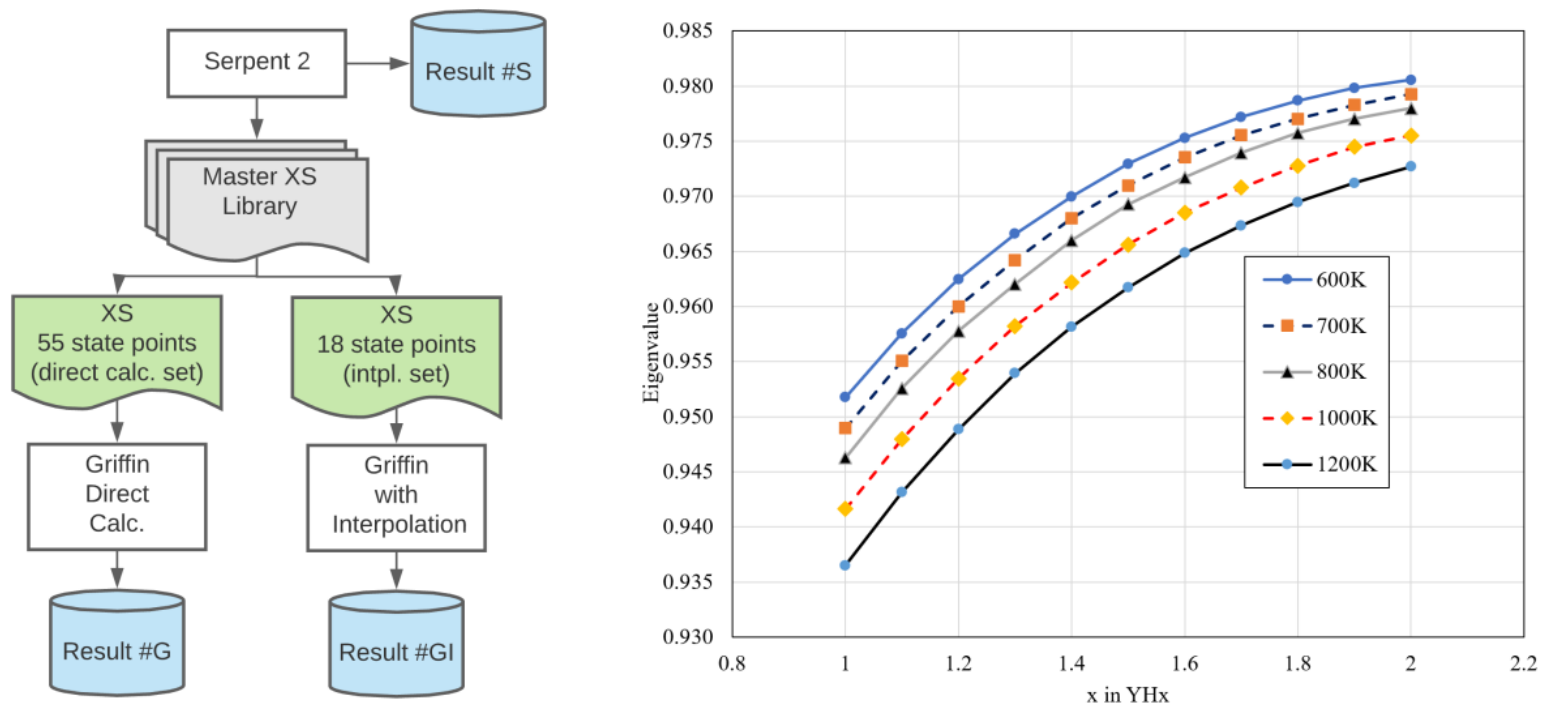

Figure A2-1. Method overview of bidimensional temperature \& H-content cross-section interpolation verification (Left) and Serpent-2 results for all grid points (Right).

To test the accuracy of the multigroup cross-sections interpolated in the MOOSE framework for multiphysics simulations, verification studies have been performed using the procedures shown in Figure A2-2. In particular, Griffin reactor physics calculations have been performed with two sets of multigroup cross sections: the "Direct" sets are cross sections directly read from the master cross section library and the "Interpolation" set are cross-sections interpolated from a smaller 2-D lookup table with 18 Serpent- 2 cases picked out of the 55 cases. Figure A2-2 shows how the 18 grid points were selected to form the lookup table for interpolating cross sections. The grid points are shown as green dots, where the grid points with fuel temperatures were set at $T=600 \mathrm{~K}, 800 \mathrm{~K}$ and $1200 \mathrm{~K}$ and hydrogen contents $\mathrm{x}$ in YHx were set at $x=1.0,1.2,1.4,1.6,1.8$ and 2.0. With this 2-D lookup table, multigroup cross sections were interpolated among the different grid points. The red dash lines shown in Figure A2-2 are the intermediate points to test the interpolated results. For instance, the cross-sections for the fuel temperature at $700 \mathrm{~K}$ were generated from grid values at $600 \mathrm{~K}$ and $800 \mathrm{~K}$. The cross-sections for the fuel temperature at $1000 \mathrm{~K}$ were tabulated from the grid values at $800 \mathrm{~K}$ and $1200 \mathrm{~K}$. Similarly, the hydrogen state points were interpolated between the green grid dots. 
Fuel

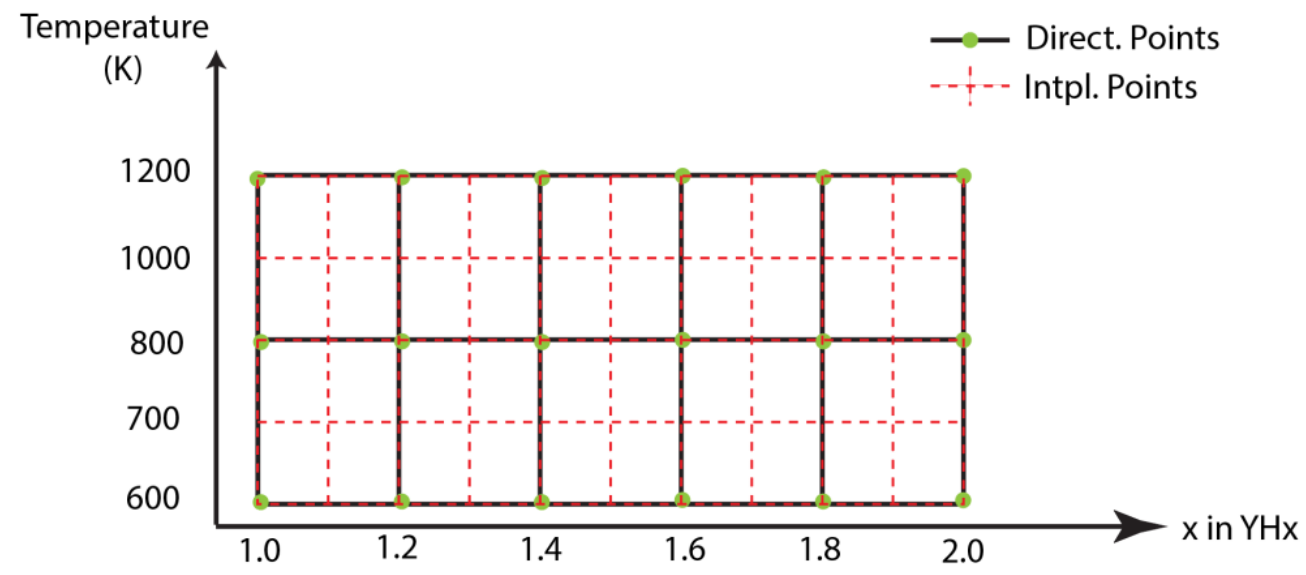

Figure A2-2. Selection of interpolation set from the master cross-section library.

\subsubsection{Results}

Griffin calculated $k_{\text {eff }}$ and the axial power distributions by using the direct cross sections or using the interpolated cross-section. Those estimates were compared for evaluating the accuracy of the interpolation method used in multiphysics simulations. The SAAF-CFEM-SN transport scheme was used in solving the neutron transport equation. Specifically, the $\mathrm{S}_{\mathrm{N}}(3,3)$ Gaussian-Chebyshev cubature with 6 directions in the polar angle and 12 directions in the azimuthal angle was used. The convergence criteria for linear iteration and non-linear iteration were selected as $1.0 \times 10^{-4}$ and $1.0 \times 10^{-5}$. Then, $\mathrm{S}_{\mathrm{N}}(3,3)$ calculations were performed at both the grid points and the interpolated points as shown in Figure A2-2. Comparing with the Serpent-2 reference results, Griffin $\mathrm{S}_{\mathrm{N}}(3,3)$ calculations overestimated the $k_{\text {eff }}$ by a similar amount for all the cases. The average difference is about $393 \mathrm{pcm}$ larger. These discrepancies can be improved with more angles added in the angular discretization or as shown later with more cross-section homogenization zones for the 3-D unit cell model.

Figure A2-3 a) compares the $\mathrm{S}_{\mathrm{N}}(3,3)$ calculated k-eff using the two different sets of cross section libraries at both the grid points and the interpolated points. They all match each other very well at all the points. The cross sections used at the grid points in the two Griffin $\mathrm{S}_{\mathrm{N}}(3,3)$ calculations were the same. Therefore, as shown in Figure A2-3 b), the $\Delta \mathrm{k}$-eff are zero at these grid points. For the interpolation points shown in Figure A2-3 b), the cross sections were interpolated among two grid values. The $\Delta \mathrm{k}$-eff at these interpolated points is within $40 \mathrm{pcm}$. The points shown in Figure A2-3 c) were all interpolated points, with multigroup cross sections obtained either using two grid values if the hydrogen content " $x$ " is on the grid or using four grid values if " $x$ " is not on the grid. As shown in this figure, the errors are usually larger for those cases interpolated among four grid values. The maximum $\Delta \mathrm{k}$-eff is within $20 \mathrm{pcm}$ among all cases at $T=700 \mathrm{~K}$, while the maximum $\Delta \mathrm{k}$-eff is $63 \mathrm{pcm}$ at $T=1000 \mathrm{~K}$ since a coarse temperature grid was used for interpolating the grid values at $1000 \mathrm{~K}$. 


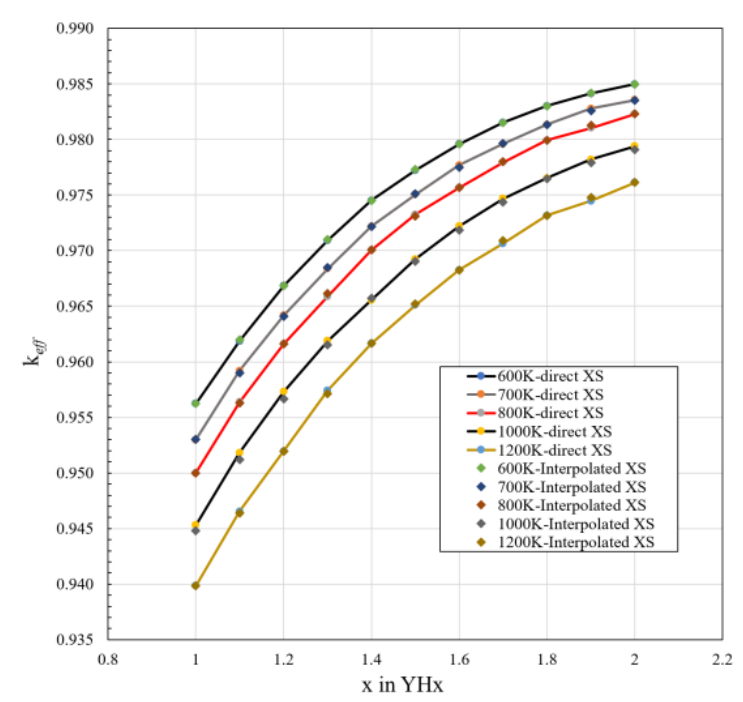

(a)

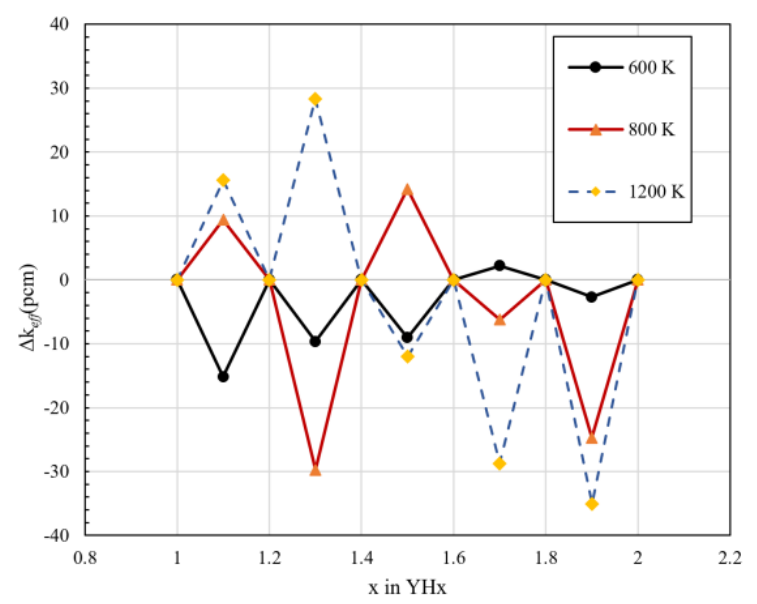

(b)

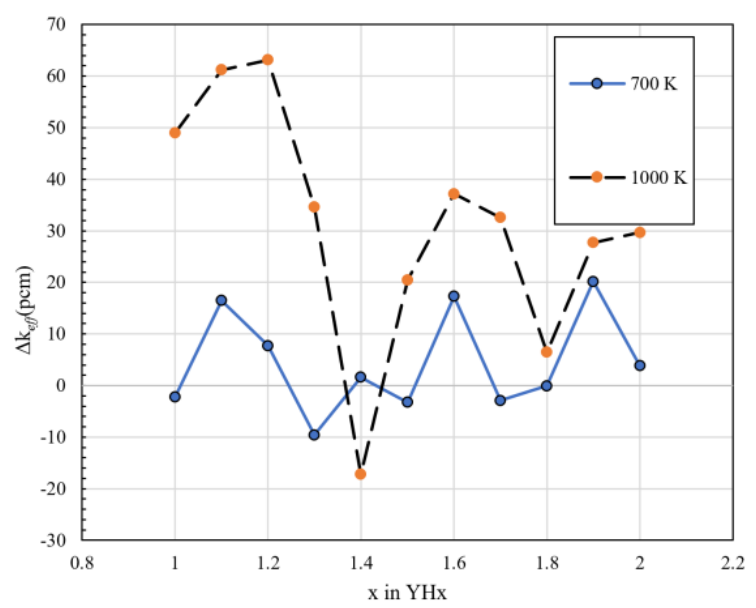

(c)

Figure A2-3. Eigenvalue comparison of the Griffin calculation with cross-sections directly converted from Serpent-2 output or with cross-sections interpolated from the 2-D look-up table:

(a) $k_{\text {eff }}$ for all grid points; (b) $\Delta k_{\text {eff }}=k_{\text {eff }}^{\text {direct }-x s}-k_{\text {eff }}^{\text {interpolated }-x s}$ for temperature grid at $600 \mathrm{~K}, 800 \mathrm{~K}$ and $1200 \mathrm{~K}$; (c) $\Delta k_{\text {eff }}$ for temperature grid at $700 \mathrm{~K}$ and $1000 \mathrm{~K}$.

With a similar approach shown in Appendix 1, the fuel temperature reactivity feedback coefficients $\alpha_{\mathrm{f}}$ can be calculated similarly among the two fuel temperature points $700 \mathrm{~K}$ and $1000 \mathrm{~K}$. Table A2-1 compared the fuel temperature reactivity feedback coefficients obtained from simulations using cross sections directly generate from Serpent-2 or using the interpolated cross sections at each $\mathrm{H}$-content point. Overall, the differences are small and ranged from $1 \%$ to about $7.7 \%$. The reactivity feedback effects due to $\mathrm{H}$-content change can also be calculated using $\mathrm{k}$-eff at its neighboring points if we define the coefficient $\alpha H$ as: $\alpha_{H}=\left(k_{e f f}^{T_{f}, x+\Delta x}-k_{e f f}^{T_{f}, x}\right) /\left(\frac{\Delta x}{x}\right)$, where $\left(\frac{\Delta x}{x}\right)$ represents the amount of $\mathrm{H}$-content changed at this grid point. As shown in Table A2-1, in general, the differences of $\alpha_{H}$ using the two different cross sections are small at most of the grid points. At a few grid points, i.e., $\mathrm{T}=1000 \mathrm{~K}$ and $\mathrm{x}=1.8$, the error is more than $10 \%$, which indicates that error cancellation might exist in this assessment analysis. Due to the coarse $\mathrm{H}$-content grid, low order $\mathrm{S}_{\mathrm{N}}$ 
solution used in the simulations, the $\Delta \mathrm{k}$-effs are always less accurate than each k-effs obtained from the numerical calculations. For future multiphysics simulations, the 2-D look-up table on the Hcontent grid axis can be refined to provide better accuracies for modeling the reactivity feedback effects from the hydrogen dissociation process.

Table A2-1. Calculated fuel temperature reactivity feedback coefficients ( $\mathrm{pcm} / \mathrm{K}$ ) and the reactivity feedback coefficient due to the H-content variation $(\mathrm{pcm} /(\Delta \mathrm{x} / \mathrm{x}))$ from $\operatorname{Griffin} \operatorname{SN}_{N}(3,3)$ method using cross sections directly from Serpent output or using the interpolated cross sections for the 3-D unit cell model.

\begin{tabular}{|c|c|c|c|c|c|c|}
\hline \multicolumn{2}{|c|}{ H-content $x$} & 1.0 & 1.1 & 1.2 & 1.3 & 1.4 \\
\hline \multicolumn{2}{|c|}{$\alpha_{f}^{\text {direct }}$} & -2.56 & -2.45 & -2.29 & -2.16 & -2.21 \\
\hline \multicolumn{2}{|c|}{$\Delta \alpha_{f}=\alpha_{f}^{\text {interpolate }}-\alpha_{f}^{\text {direct }}$} & -0.17 & -0.15 & -0.19 & -0.15 & 0.06 \\
\hline \multicolumn{2}{|c|}{$\Delta \alpha_{f} / \alpha_{f}^{\text {direct }}(\%)$} & 6.2 & 5.8 & 7.7 & 6.5 & -2.8 \\
\hline \multirow{3}{*}{ Fuel $\mathrm{T}=700 \mathrm{~K}$} & $\alpha_{H}^{\text {direct }}$ & $6.20 \mathrm{E}+03$ & $5.49 \mathrm{E}+03$ & $5.03 \mathrm{E}+03$ & $4.94 \mathrm{E}+03$ & $4.04 \mathrm{E}+03$ \\
\hline & $\Delta \alpha_{H}^{*}$ & -188.0 & 96.7 & 208.0 & -146.0 & 68.2 \\
\hline & $\Delta \alpha_{H} / \alpha_{H}^{\text {direct }}(\%)$ & -3.03 & 1.76 & 4.13 & -2.96 & 1.69 \\
\hline \multirow{3}{*}{$\begin{array}{c}\text { Fuel } \mathrm{T}=1000 \\
\mathrm{~K}\end{array}$} & $\alpha_{H}^{\text {direct }}$ & $6.52 \mathrm{E}+03$ & $6.02 \mathrm{E}+03$ & $5.50 \mathrm{E}+03$ & $4.76 \mathrm{E}+03$ & $5.18 \mathrm{E}+03$ \\
\hline & $\Delta \alpha_{H}{ }^{*}$ & -122.0 & -21.0 & 342.0 & 674.0 & -528.0 \\
\hline & $\Delta \alpha_{H} / \alpha_{H}^{\text {direct }}(\%)$ & -1.88 & -0.35 & 6.22 & 14.15 & -10.18 \\
\hline \multicolumn{2}{|c|}{ H-content $x$} & 1.5 & 1.6 & 1.7 & 1.8 & 1.9 \\
\hline \multicolumn{2}{|c|}{$\alpha_{f}^{\text {direct }}$} & -1.94 & -1.83 & -1.64 & -1.61 & -1.52 \\
\hline \multicolumn{2}{|c|}{$\Delta \alpha_{f}=\alpha_{f}^{\text {interpolate }}-\alpha_{f}^{\text {direct }}$} & -0.08 & -0.06 & -0.12 & -0.02 & -0.03 \\
\hline \multicolumn{2}{|c|}{$\Delta \alpha_{f} / \alpha_{f}^{\text {direct }}(\%)$} & 4.0 & 3.2 & 6.8 & 1.2 & 1.9 \\
\hline \multirow{3}{*}{ Fuel $\mathrm{T}=700 \mathrm{~K}$} & $\alpha_{H}^{\text {direct }}$ & $3.93 \mathrm{E}+03$ & $3.07 \mathrm{E}+03$ & $2.95 \mathrm{E}+03$ & $2.60 \mathrm{E}+03$ & $1.44 \mathrm{E}+03$ \\
\hline & $\Delta \alpha_{H} *$ & -308.0 & 323.0 & -47.9 & -365.0 & 311.0 \\
\hline & $\Delta \alpha_{H} / \alpha_{H}^{\text {direct }}(\%)$ & -7.84 & 10.51 & -1.62 & -14.02 & 21.60 \\
\hline \multirow{3}{*}{$\begin{array}{c}\text { Fuel } \mathrm{T}=1000 \\
\mathrm{~K}\end{array}$} & $\alpha_{H}^{\text {direct }}$ & $4.43 \mathrm{E}+03$ & $3.97 \mathrm{E}+03$ & $3.13 \mathrm{E}+03$ & $3.05 \mathrm{E}+03$ & $2.23 \mathrm{E}+03$ \\
\hline & $\Delta \alpha_{H} *$ & -251.0 & 74.1 & 443.0 & -382.0 & -37.2 \\
\hline & $\Delta \alpha_{H} / \alpha_{H}^{\text {direct }}(\%)$ & -5.66 & 1.87 & 14.15 & -12.55 & -1.67 \\
\hline
\end{tabular}

$* \Delta \alpha_{H}=\alpha_{H}^{\text {interpolate }}-\alpha_{H}^{\text {direct }}$ 
In addition, as shown in Figure A2-4, the axial power distributions obtained from the verification study were also compared. The reference power densities were tallied with in the 3-D unit cell using a $5 \mathrm{~cm}$ axial mesh. The power densities in the Griffin $\mathrm{S}_{\mathrm{N}}(3,3)$ calculations were obtained from the Griffin postprocess directly at each axial material block. Figure A2-4 a) first compared the Griffin calculated axial power distribution with the Serpent-2 reference result within the 3-D unit cell model at fuel temperature $T=1200 \mathrm{~K}$ and hydrogen content $x=1.4$, where the cross sections are directly obtained from Serpent-2 simulations. Excellent agreement is observed except at the first centimeters of the fuel pins closer to the bottom Beryllium reflector. Figure A2-4 b) shows the normalized axial power distributions obtained from $\operatorname{Griffin} \mathrm{S}_{\mathrm{N}}(3,3)$ calculations with either the direct cross-sections or the interpolated cross-sections at $T=1000 \mathrm{~K}$ and $x=1.1$. They match extremely well with the maximum difference less than $0.05 \%$. The axial distributions at other grid points or interpolated points were all matched well. Figure A2-4 c) and d) show the maximum relative differences among the axial locations for each of cases. The maximum differences are all smaller than $0.1 \%$. Similarly, the differences in the axial power distributions are also slightly larger with fuel temperature at $1000 \mathrm{~K}$ than those with fuel temperature at $700 \mathrm{~K}$ due to a coarser temperature grid used in the cross-section interpolations.

Overall, the numerical simulations performed in this study showed that the bidimensional cross section tabulation method in the MOOSE multiphysics simulation framework can be used to accurately model the reactor physics in the transient simulations.

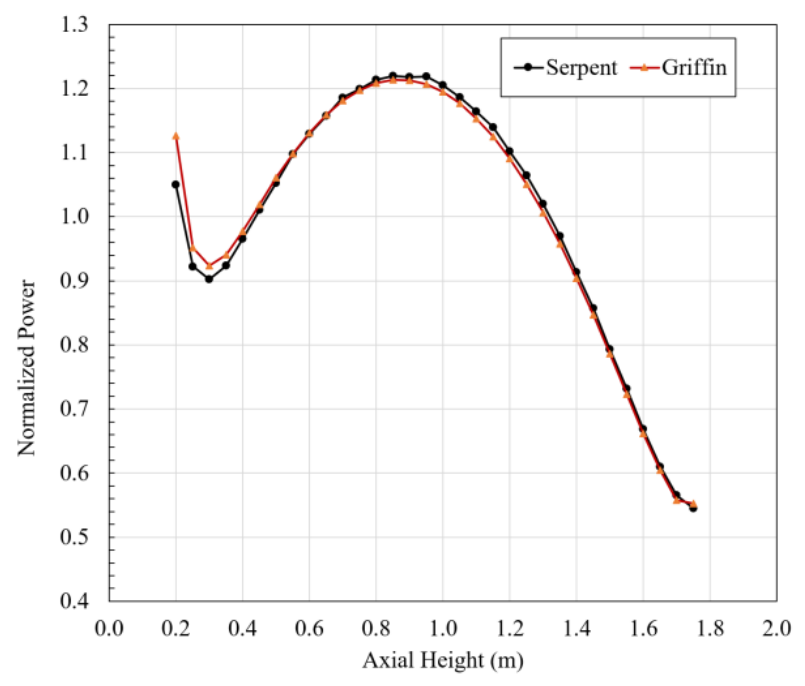

(a)

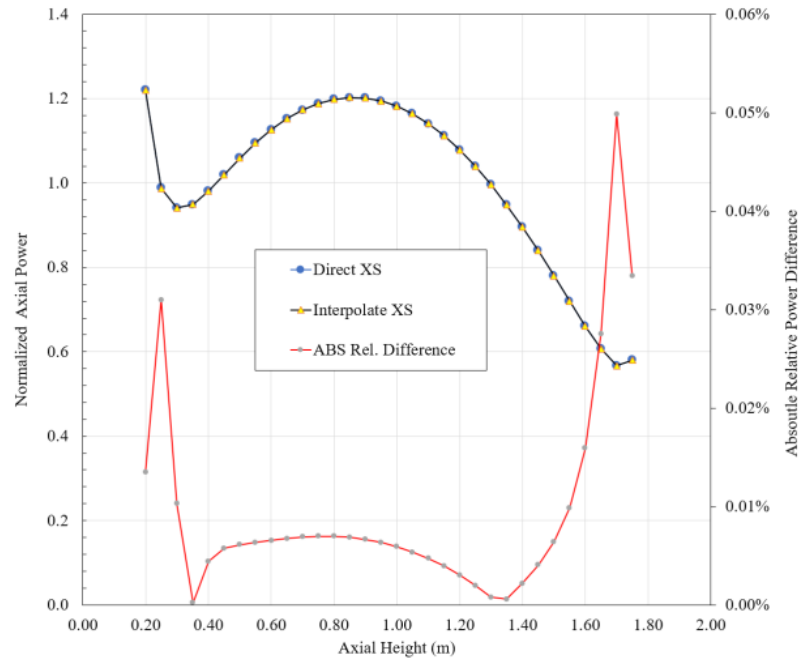

(b) 


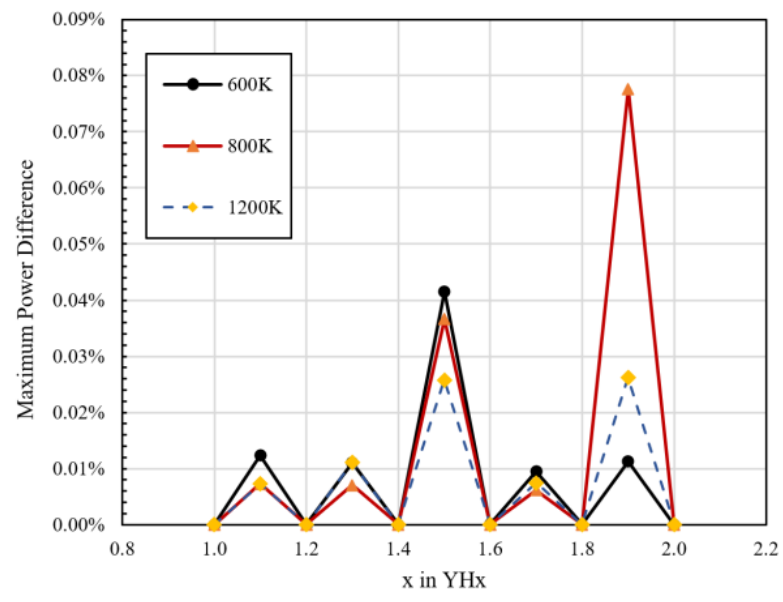

(c)

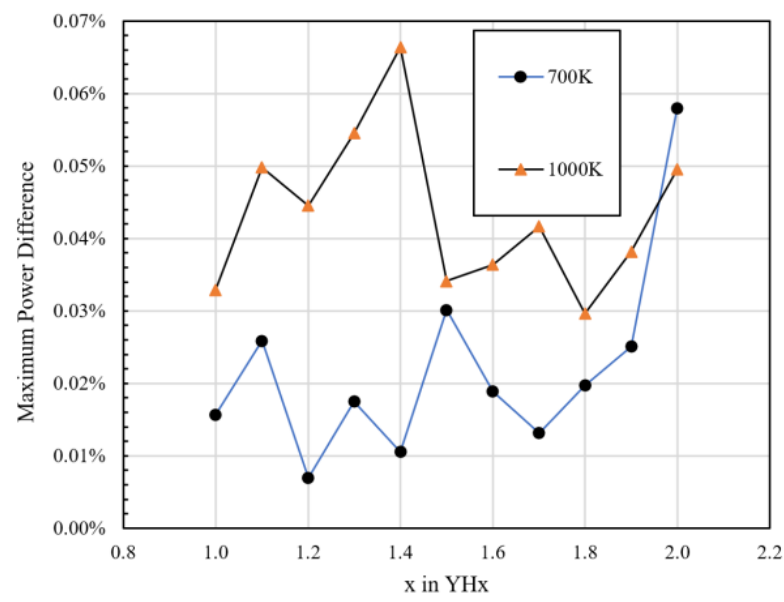

(d)

Figure A2-4. Axial power comparison of the Griffin calculation with cross-sections directly converted from Serpent-2 output or with cross-sections interpolated from 2-D look-up table: a)

Serpent-2 normalized axial power compared with Griffin power at $\mathrm{T}=1200 \mathrm{~K}$ and $\mathrm{x}=1.4$; $\mathrm{b}$ ) normalized axial distributions for case with $\mathrm{T}=1000 \mathrm{~K}$ and $\mathrm{x}=1.1$; c) maximum relative differences of axial power for temperature grid at $600 \mathrm{~K}, 800 \mathrm{~K}$ and $1200 \mathrm{~K}$; d) maximum relative differences of axial power for temperature grid at $700 \mathrm{~K}$ and $1000 \mathrm{~K}$.

\subsubsection{Spectral Effects in Multigroup Cross-section Generation}

The Griffin simulations use cross-sections homogenized from Serpent-2 simulations on an 11energy group structure. These cross-sections were flux weighted within each material region in the 3-D unit cell model and were used in every finite element mesh of the material block:

$$
\Sigma_{i, g}=\frac{\int_{E_{g}}^{E_{g-1}} d E \iint d V d \Omega \Sigma_{i}(r, E, \Omega) \phi(r, E, \Omega)}{\int_{E_{g}}^{E_{g-1}} d E \iint d V d \Omega \phi(r, E, \Omega)}
$$

The homogenized multigroup cross-sections over the whole unit-cell region might not always be suited for regions where the neutron spectrum is significantly different from the average neutron spectrum within the model. For instance, the neutron spectrum at the end of fuel pin region will be much softer than the neutron spectrum at the center fuel pin region. A simple numerical study was performed to examine the spectral effects in this 3-D unit-cell model.

In particular, in the Serpent-2 model, the 3-D unit cell active fuel region was divided into four axial layers, with the top and bottom $10 \mathrm{~cm}$ of the fuel pins forming two separate layers and the middle fuel section also being evenly dived into two layers each of length $70 \mathrm{~cm}$. The material compositions and temperatures were the same in all the four layers. Then multigroup cross-sections were homogenized for each of the material blocks at the four axial layers and were converted to the ISOXML format. Similarly, the Griffin $\mathrm{S}_{\mathrm{N}}(3,3)$ transport solver was applied based on the updated cross-section sets.

Figure A2-5 compares the calculated k-effs with the updated cross-section sets with the serpent-2 reference results and the Griffin $S_{N}(3,3)$ calculation using previous one region homogenized cross- 
sections. In these cases, the fuel temperature is at $600 \mathrm{~K}$, and $\mathrm{x}$ in the $\mathrm{YHx}$ varies from 1.0 to 2.0. Overall, better agreement is observed between the Griffin $\mathrm{S}_{\mathrm{N}}(3,3)$ calculations to the Serpent-2 reference results by homogenizing the cross sections at multiple axial layers. However, the improvement is not uniform. The $\Delta \mathrm{k}$-eff is reduced by less than $15 \mathrm{pcm}$ at $\mathrm{x}=1.7$, but is reduced by $220 \mathrm{pcm}$ at $\mathrm{x}=1.1$, indicating there might be error cancellations in these numerical simulations. More studies with finer finite element meshes and more angles used in the $\mathrm{S}_{\mathrm{N}}$ transport solver might help obtaining more consistent results in the future.

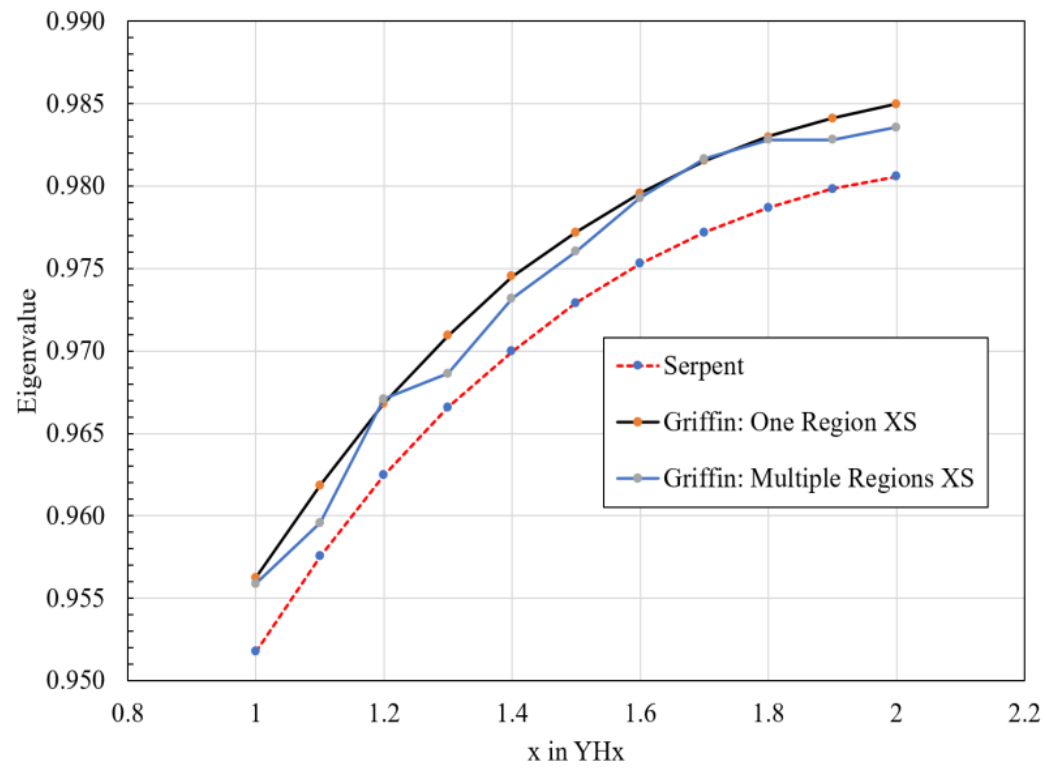

Figure A2-5. Comparison of the calculated Griffin $\mathrm{S}_{\mathrm{N}}(3,3) \mathrm{k}$-effs using one region homogenized cross-sections or multiple region homogenized cross-sections with the Serpent-2 reference results with fuel material at $600 \mathrm{~K}$ in the 3-D unit cell.

\subsection{Heterogeneity Effects in Multigroup Cross-section Generation}

\subsubsection{Assessment procedures}

In a more refined reactor model, the materials properties are also varying spatially. For instance, in the multiphysics simulations performed for our 3-D unit-cell model, the temperatures and hydrogen contents were different at each mesh block. Their values were determined from other physics calculations, i.e., fuel and moderator temperatures determined from BISON and Sockeye coupled simulations which models the heat transfer and thermal-mechanics in the unit-cell, and the hydrogen contents determined from the SWIFT code which models the hydrogen dissociation process. However, in the Serpent-2 numerical models for homogenizing the multigroup crosssections, the materials within the 3-D model are usually assumed to be uniform at certain grid points with pre-determined temperatures and hydrogen content ("x" in YHx). A simple numerical study was also performed to check if these heterogeneity effects in the multiphysics simulations which were not considered in the multigroup cross sections will lead to significant discrepancies on the reactor physics calculations.

Particularly, three numerical tests were performed as shown in Figure A2-6 where each of them has different fuel temperatures and hydrogen contents at four axial zones in the 3-D unit cell model. Two approaches have been used to generate the multigroup cross sections for these models. The 
first approach is the one used in the regular multiphysics simulations, where the multigroup cross sections were interpolated from a 2-D lookup table with grid values generated from Serpent-2 uniform models at different grid points. To make sure the comparison is fair, four axial layers were used in these uniform Serpent-2 models to allow the cross-section homogenized for each of the four axial zones. The second approach is the so called "direct" approach in which the multigroup crosssections directly homogenized from the Serpent-2 model which has all the heterogeneous zones explicitly modeled. The three test cases shown in Figure A2-6 were selected so that the first two cases had only one variable (fuel temperature or $\mathrm{x}$ in the hydrogen contents) changing in the different axial layers, and the third case had both variables changing in the different axial layers.

\begin{tabular}{|c|c|c|}
\hline Reg. \# 1 & Reg. \# 1 & Reg. \# 1 \\
\hline $\mathrm{H}=2.00$ & $\mathrm{H}=2.00$ & $\mathrm{H}=2.00$ \\
\hline @ 600K & @ 650K & @ 650K \\
\hline Reg. \# 2 & Reg. \# 2 & Reg. \# 2 \\
\hline $\mathrm{H}=1.65$ & $\mathrm{H}=2.00$ & $H=1.65$ \\
\hline (a)600K & @ 800K & @ 800K \\
\hline Reg. \# 3 & Reg. \# 3 & Reg. \# 3 \\
\hline $\mathrm{H}=1.35$ & $\mathrm{H}=2.00$ & $\mathrm{H}=1.35$ \\
\hline @600K & @ 950K & @ 950K \\
\hline Reg. \# 4 & Reg. \# 4 & Reg. \# 4 \\
\hline $\mathrm{H}=1.00$ & $\mathrm{H}=2.00$ & $\mathrm{H}=1.00$ \\
\hline @ 600K & @ 1150K & (a) 115 \\
\hline
\end{tabular}

Figure A2-6. Interpolation of varied material cross-section assessment cases settings.

\subsubsection{Results}

Table A2-2 shows the Griffin $\mathrm{S}_{\mathrm{N}}(3,3)$ calculated k-effs by using the two sets of multigroup crosssections. For all three cases, there are about a few tens or about a hundred pcm differences for the calculated k-effs using those interpolated cross-sections (without the heterogeneity effects included in the Serpent-2 model), which is small when compared to the overall change of reactivity considered. As shown in Figure A2-7, the axial power distributions matched very well at most of the axial locations. The maximum differences are all less than $6 \%$ for the three cases and are at the bottom first $10 \mathrm{~cm}$ axial layer. Similar large errors at the same locations were also observed while comparing the Griffin $\mathrm{S}_{\mathrm{N}}(3,3)$ calculated power distributions with the Serpent-2 reference result.

Table A2-2. Result comparisons between interpolated cross-section approach and direct crosssection approach for three cases.

\begin{tabular}{|c|c|c|c|c|}
\hline $\begin{array}{c}\text { Model } \\
\text { settings }\end{array}$ & $\begin{array}{l}\text { XS generation } \\
\text { procedure }\end{array}$ & k-eigenvalue & $\begin{array}{c}\text { k-eigenvalue } \\
\text { diffference }(\mathrm{pcm})\end{array}$ & $\begin{array}{c}\text { Max axial power } \\
\text { difference }(\%)\end{array}$ \\
\hline \multirow{2}{*}{ Case \# 1} & Interpolated XS & 0.97819 & \multirow{2}{*}{64.6} & \multirow{2}{*}{4.94} \\
\hline & Direct XS & 0.97754 & & \\
\hline \multirow{2}{*}{ Case \# 2} & Interpolated XS & 0.98185 & \multirow{2}{*}{-92.6} & \multirow{2}{*}{4.42} \\
\hline & Direct XS & 0.98278 & & \\
\hline \multirow{2}{*}{ Case \# 3} & Interpolated XS & 0.97338 & \multirow{2}{*}{-43.0} & \multirow{2}{*}{5.88} \\
\hline & Direct XS & 0.97381 & & \\
\hline
\end{tabular}



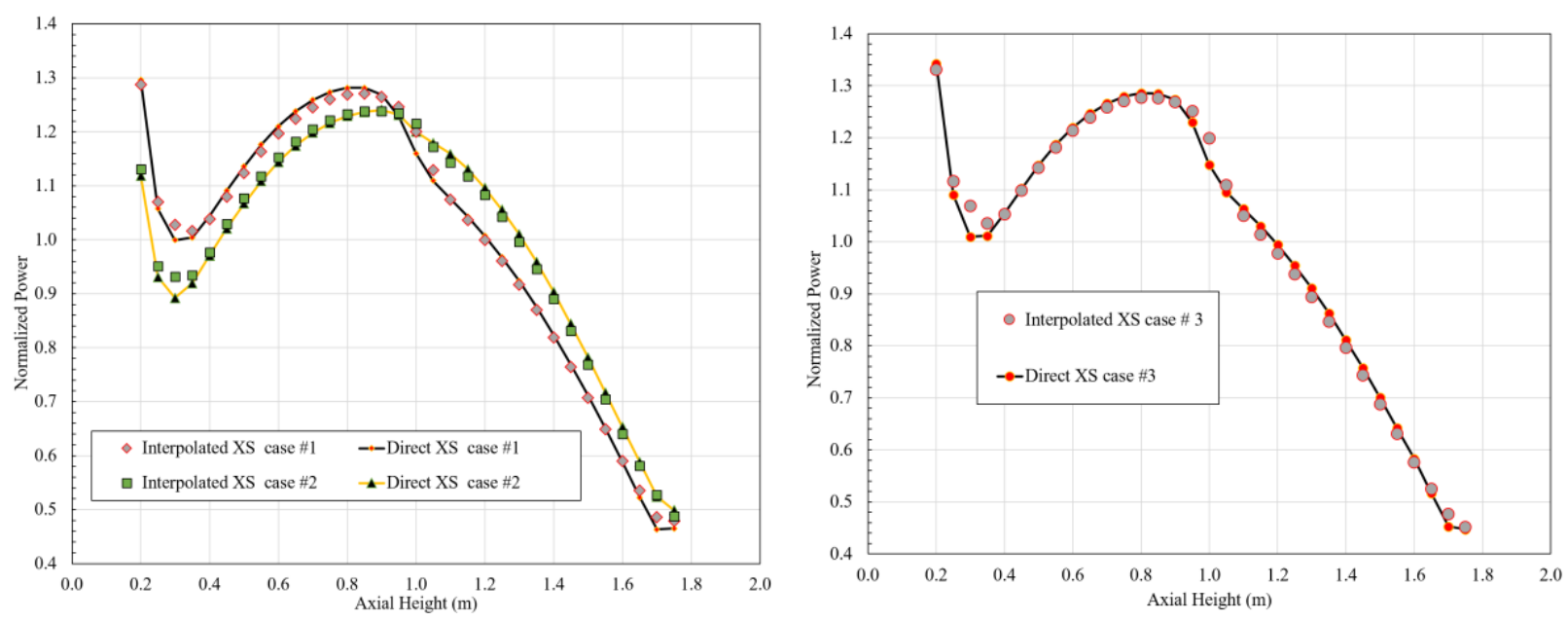

Figure A2-7. Axial power distribution in the fuel pin comparison between direct cross-section and interpolated cross-section calculation of case \#1, case \#2 (Left) and case \#3 (Right).

\subsection{Conclusion}

A dedicated study has been performed to study the accuracy of the piecewise linear interpolation scheme which has been used to model the temperature dependence and hydrogen content dependence of multigroup cross-sections in our 3-D unit-cell multiphysics transient simulations. This numerical analysis showed that with a careful set 2-D look-up table obtained from the MC Serpent-2 simulations, the reactor physics within the 3-D unit-cell model can be calculated accurately with this interpolate scheme.

The impact on the reactivity feedback from the cross-section interpolation on fuel, moderator and hydrogen content has been provided, which is important to the capture the correct feedback to multiphysics simulation. The result analysis showed that with the carefully prepared look-up tables, the reactivity coefficients obtained from the interpolated cross-section set can accurately capture the reactivity feedback from both temperature and hydrogen content.

Additional numerical study has also been performed to examine the spectral effects in condensing the multigroup cross sections. This analysis suggested that condensing the multigroup cross sections at multiple regions where the neutron spectrum might be significantly different can improve the accuracy of the Griffin reactor physics calculations.

In addition, in multiphysics simulations, the heterogeneity effects in the material properties (different fuel temperature or hydrogen contents at different regions) were ignored while generating the multigroup cross sections. This numerical study examined three particular cases, which all have distributed fuel temperatures or hydrogen contents. Results showed good agreement in terms of eigenvalue and power distribution when comparing the Griffin results using interpolated or directly obtained sets of cross-sections. 
Argonne

Nuclear Science and Engineering Division

Argonne National Laboratory

9700 South Cass Avenue, Bldg. 208

Argonne, IL 60439

www.anl.gov

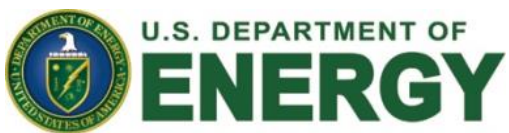

Argonne National Laboratory is a U.S. Department of Energy

laboratory managed by UChicago Argonne, LLC 